\title{
SUPER-RADIANT DYNAMICS, DOORWAYS, AND RESONANCES IN NUCLEI AND OTHER OPEN MESOSCOPIC SYSTEMS
}

\author{
Naftali Auerbach ${ }^{1,2}$ and Vladimir Zelevinsky ${ }^{2,3}$ \\ ${ }^{1}$ School of Physics and Astronomy, Tel Aviv University, Tel Aviv, 69978, Israel \\ ${ }^{2}$ Department of Physics and Astronomy, Michigan State University, \\ East Lansing, Michigan 48824-2320, USA \\ ${ }^{3}$ National Superconducting Cyclotron Laboratory, Michigan State University, \\ East Lansing, Michigan 48824-1321, USA
}

\begin{abstract}
The phenomenon of super-radiance (Dicke effect, coherent spontaneous radiation by a gas of atoms coupled through the common radiation field) is well known in quantum optics. The review discusses similar physics that emerges in open and marginally stable quantum many-body systems. In the presence of open decay channels, the intrinsic states are coupled through the continuum. At sufficiently strong continuum coupling, the spectrum of resonances undergoes the restructuring with segregation of very broad super-radiant states and trapping of remaining long-lived compound states. The appropriate formalism describing this phenomenon is based on the Feshbach projection method and effective non-Hermitian Hamiltonian. A broader generalization is related to the idea of doorway states connecting quantum states of different structure. The method is explained in detail and the examples of applications are given to nuclear, atomic and particle physics. The interrelation of the collective dynamics through continuum and possible intrinsic many-body chaos is studied, including universal mesoscopic conductance fluctuations. The theory serves as a natural framework for general description of a quantum signal transmission through an open mesoscopic system.
\end{abstract}


1. Introduction

2. Origin of super-radiance

- Original idea

- Transport through a lattice

3. Open quantum system

- Projection method and effective non-Hermitian Hamiltonian

- Effective Hamiltonian and reaction theory

- Emergence of resonances

- Overlapping resonances and super-radiance

4. Nuclear continuum shell model

- Two-level example

- Realistic calculations

- Example: Oxygen isotopes

- Some technicalities

5. Doorway states

- Dynamics based on doorway states

- The case of a single doorway

- When is this approach valid?

- Isobaric analog resonances

- Giant resonances

- Several channels and intermediate structure

- Highly excited doorway state

- Additional comments

6. Other applications

- Atomic and molecular physics

- Intermediate and high energy physics

- The $\bar{N} N$ states

- The $\Delta$-nucleon system

- High energy phenomena

7. Quantum chaos in an open system

- Many-body quantum chaos 
- Statistics of resonances

- Statistics of resonance widths

- Role of intrinsic chaos

8. Signal transmission through a mesoscopic system

- Common physics of mesoscopic systems

- Statistics of cross sections

- Fluctuations of cross sections

- From Ericson to conductance fluctuations

9. Conclusion and outlook 


\section{Introduction}

More than half a century ago Robert Dicke published an article where he predicted the phenomenon of super-radiance [1]. This phenomenon was indeed experimentally observed in an optically pumped gas [2] and later studied in detail and widely used in quantum optics [3, 4] as well in physics of plasmas and beam physics 5 . The super-radiance was also observed with the free-electron laser [6] and with atomic matter waves, including Bose-Einstein condensate [7. The quantum-mechanical essence of super-radiance in the original formulation is relatively simple. Two-level systems ("atoms" or "qubits") can be coupled even in the absence of their direct interaction. The coupling proceeds through the common radiation field. The migrating resonant excitation combines the atoms in a collective capable of releasing the burst of radiation as a whole. The phenomenon therefore is called coherent spontaneous radiation that sounds as an oxymoron since traditionally the spontaneous radiation was considered as being incoherently produced by individual atoms.

In the end of the nineteen eighties it was understood that the physics underlying super-radiance is much more general and can find broad applications in various regions of the quantum world. The necessary ingredient is the set of unstable quantum states with the same exact quantum numbers. The states should be capable of decaying into a common decay channel (or few channels). This creates an analog of their coupling through the common "field of radiation", or common continuum; the coupling becomes effective when the widths of individual adjacent states grow and overlap. The system of overlapping resonances undergoes a sharp restructuring forming super-radiant states, one for each common open channel. In the ideal version of quantum optics, when the wavelength of radiation significantly exceeds the size of the system, the identical atoms have identical levels and widths which amplifies the effect. In a general case, the levels are not degenerate, and the super-radiant transition can happen only when the typical widths are of the order of typical spacings between the unstable levels.

The mechanism of super-radiance is omnipresent being simply a consequence of quantum mechanics. It was seen in the problems of quantum chemistry, atomic and molecular physics. In a pure form it was discovered in nuclear physics in consideration of the decay of giant resonances 8 when the numerical simulation clearly indicated the appearance of very broad resonances for each open decay channel when the continuum coupling exceeds a critical strength. At the same time the remaining resonances are getting more narrow signaling the existence of trapped states with long lifetime.

The physical and mathematical analogy of this behavior of a set of resonances to the super-radiance phenomenon was first indicated in [9, 10]. The starting point there was quantum chaos, namely a question of the influence of the continuum coupling on the statistical distribution of the level spacings. It turns out that the super-radiant behavior survives the intrinsic dynamical chaos being mainly determined by the character of decay. The spectral statistics, typical for canonical ensembles of random matrices, evolve into the statistics of 
complex energies which are at the same time the poles of the scattering matrix in the space of open channels. The statistical properties, in turn, are instrumental in defining the fluctuations and correlations of cross sections of various reactions proceeding through the channels open in the energy interval under study. The phenomenon of super-radiance reveals itself in a specific two-cloud distribution of energies and widths of resonances in the complex plane [11, 12.

The adequate mathematical formalism for the description of this physics is provided by the effective non-Hermitian Hamiltonian [13, suggested in nuclear theory. The method goes back to Feshbach [14, 15] and Fano [16] extending and improving (eliminating not well defined channel radii) the boundary condition method originated by Kapur and Peierls [17, Wigner and Eisenbud [18, and Teichmann and Wigner 19. Similar ideas can be found already in much earlier notebooks by Majorana 20]. The classical review articles are available concerning this period of development [21, 22, 23]. The equivalent method was independently developed and applied, among other problems, to quantum scattering theory by the outstanding Russian-Israeli mathematician M.S. Livšic [24, 25].

This approach was described in the well known book [26] although the fact that the super-radiant physics directly follows from this Hamiltonian was understood only later. The effective Hamiltonian, in general energy-dependent, can be derived by a standard projection method when the part of the dynamics corresponding to the continuum channels is formally eliminated and the whole Schrödinger equation is projected onto the internal dynamics. In this part of Hilbert space the non-Hermiticity appears as a result of the presence of open channels so that the eigenstates above threshold become unstable resonances, and the eigenvalues of the Hamiltonian are complex energies $\mathcal{E}=E-(i / 2) \Gamma$, where $\Gamma$ is the width of the resonance. The anti-Hermitian part of the Hamiltonian has a specific factorized structure as follows from the unitarity of the dynamics in the full space [27. In the regime of strong continuum coupling this part plays the main role and the rank of the corresponding matrix, equal to the number of open channels, determines the number of super-radiant states. The Hermitian part of the effective Hamiltonian is responsible for energy shifts of resonances and bound states; by its nature it is similar to the Lamb shift but in this case of collective origin. It is useful to stress that the non-Hermitian character of the Hamiltonian here is not related to any approximation as long as all channels are accounted for.

In our review, the formalism of the effective Hamiltonian will serve as a foundation of one of the most effective approaches to the description of open many-body systems. This is especially important for modern nuclear physics where the center of interest moved to nuclei far from stability. Such nuclei are often only marginally stable being close to the drip lines which delineate the limits of existence for nuclear systems. The proximity of the continuum - both actual decay channels and virtual intermediate states - determines the specific physics of such systems. The continuum shell model [28, 29, 30, 31] developed in the framework of the effective Hamiltonian allows one to describe in a unified approach bound states, resonances and reaction cross sections. In distinction to 
other perspective approaches to loosely bound nuclei, such as the Gamow shell model, that uses the analytical continuation to the complex plane [32, 33, 34, here one can consider all processes at real energy of the reaction. The energy dependence of the effective Hamiltonian reflects, in particular, the existence of channel thresholds, and the proper behavior of the reaction amplitudes at the thresholds is a noticeable and practically important feature of the continuum shell model.

Nuclear physics is only one, even if essential, area of applications of the ideas of the effective Hamiltonian and super-radiance. In fact, this is a convenient way to describe dynamics of any quantum system in the energy region including both bound states and continuum. Atomic autoionizing states [35. and intermolecular processes [36] were long ago shown to reveal a similar behavior. Furthermore, we can expect the same trends to manifest themselves in physics of intermediate and high energy [37, 38, 39. The role of open channels in various situations can also be played by the doorway states [40] which are forming a bottleneck connecting the states of different nature [41; this is another generalization of the ideas of super-radiance.

Important applications of the super-radiance and related theory can be found in mesoscopic physics. The universal mesoscopic conductance fluctuations [42] share significant similarity with Ericson fluctuations [43, 44, typical for nuclear reactions observed in the energy region of overlapping resonances 45. The detailed analysis of correlations and fluctuations of cross sections for different channels can shed new light on the fundamental problem of the signal transmission through a quantum system, from simplest qubits to very complex molecules and artificial structures with interfering propagation paths. The first results modifying the Ericson theory by the effects of super-radiance and dependence of certain observables on the degree of internal chaoticity appeared recently in the literature [46, 47, 48], along with the applications to ordered and disordered nanostructures [49, 50]. Vice versa, the intrinsic chaos is strongly modified by the openness of the system.

Our review is devoted to the manifestations of the super-radiant mechanism outside of quantum optics. The presentation is organized as follows. We start with illustrations of the phenomenon of super-radiance in simple cases, Chapter 2. In Chapter 3 we introduce the projection formalism, derive the effective Hamiltonian and show how the generalized super-radiance emerges as a result of the strong coupling of the intrinsic states to the continuum channels. As the first application of the effective Hamiltonian we consider the nuclear continuum shell model. Chapter 4 contains examples showing this approach at work. In Chapter 5 we show the extension of the approach to doorway states and such collective excitations as giant resonances. Other examples of the same approach, Chapter 6 , will illustrate applications to different areas of physics. We devote Chapter 7 to the problems of quantum chaos in open systems and their manifestations in spectral statistics and reaction cross sections. Finally, Chapter 8 will discuss the rapidly developing area of quantum signal transmission from the same general viewpoint. The conclusion will be used mainly to list the interesting problems which are still waiting for their explorer. We will try to explain the main physical 
ideas along with their mathematical formulations but we will not be able to go through detailed calculations referring instead to the original literature. 


\section{Origin of super-radiance}

\subsection{Original idea}

For a simple start we will go back to the original idea of optical super-radiance [1] and stress the elements which later will be generalized to similar phenomena in various subfields of physics. Let us consider an idealized system of $N$ identical two-level atoms confined to the volume of a size much smaller than the wavelength of radiation that corresponds to the transition between the levels of each qubit. We can describe the intrinsic states $|\Psi\rangle$ of such a system with $N_{a}$ atoms in the ground state and $N_{b}=N-N_{a}$ atoms in the excited state with the aid of corresponding creation, $a^{\dagger}$ and $b^{\dagger}$, and annihilation, $a$ and $b$, operators as

$$
\left|\Psi\left(N_{a}, N_{b}\right)\right\rangle \equiv\left|N_{a}, N_{b}\right\rangle=\frac{1}{\sqrt{N_{a} ! N_{b} !}}\left(b^{\dagger}\right)^{N_{b}}\left(a^{\dagger}\right)^{N_{a}}|0\rangle,
$$

where for simplicity we assume that the atoms are bosons and $|0\rangle$ denotes the vacuum state.

Neglecting the radiation from the excited level, we have stable intrinsic space spanned by the states (1). It is useful to apply the Schwinger representation of angular momentum [51] with the aid of individual spins $s_{i}=1 / 2$ corresponding to each qubit and the total spin $\mathbf{S}=\sum_{i} \mathbf{s}_{i}$ of the entire system that is usually called here the Bloch vector. The identification of quantum numbers is obvious:

$$
\left|\Psi\left(N_{a}, N_{b}\right)\right\rangle \Rightarrow|S M\rangle, \quad S=\frac{1}{2}\left(N_{a}+N_{b}\right)=\frac{1}{2} N, \quad M=S_{z}=\frac{1}{2}\left(N_{b}-N_{a}\right),
$$

or, in terms of original atomic operators,

$$
|S M\rangle=[(S+M) !(S-M) !]^{-1 / 2}\left(b^{\dagger}\right)^{S+M}\left(a^{\dagger}\right)^{S-M}|0\rangle .
$$

The Hamiltonian of the closed system is trivial,

$$
H=\omega \sum_{i} s_{i z}=\omega M
$$

where the origin of the energy scale is set at the middle between the excited and ground levels of an atom.

Now we make the excited atoms unstable introducing the radiation field. If $A^{*}$ and $A$ are, in appropriate units, the amplitudes of radiation and absorption of the photon, respectively, this part of the Hamiltonian is

$$
H^{\prime}=A^{*} a^{\dagger} b+A b^{\dagger} a=A^{*} S_{-}+A S_{+},
$$

where, using the long wave limit, we neglect the phase differences between the atoms. Each act of radiation brings $M$ by one step lower. If all atoms are initially excited, $S=M=N / 2$, the Bloch vector is at the north pole of the Bloch sphere, and only spontaneous radiation of each atom is possible with the total probability $\left|A^{*}\left\langle S, S-1\left|S_{-}\right| S, S\right\rangle\right|^{2}=|A|^{2} 2 S=|A|^{2} N$. This is an 
incoherent process of independent radiation by excited atoms with the intensity proportional to their number.

However, the same Hamiltonian (5) describes also the processes of interaction between the atoms though virtual radiation and absorption of quanta. In this idealized model, such processes are amplified by the exact degeneracy of atomic levels all tuned in resonance. While the spontaneous radiation brings the average level of excitation down and the Bloch vector deviates from the vertical position, the amplitude of radiation grows through the virtual coupling between the radiators that becomes possible due to the devastation of large- $M$ states and opening possibility of the virtual absorption. The maximum is reached at $M=0$ (the Bloch vector in the equatorial plane) being proportional to $|A|^{2} S(S+1)$. Here we have a coherent spontaneous radiation - super-radiance - with the probability $\propto N^{2}$. Such a burst of spontaneous radiation was observed in optically pumped HF [2] and later studied in numerous experiments. The underlying dynamics of the Bloch vector can be presented also in a language of classical mechanics 52 .

Of course, the practical implementation is not identical to this oversimplified model. The different spatial positions of the atoms bring in the phase differences between interfering radiators. (In fact, the seemingly opposite limit of the size large compared to the wave length allows one to measure the so-called collective Lamb shift of the radiation line [53]; even a very small shift of this nature can limit accuracy of atomic clocks [54). The local nonuniformity and collisional broadening lift the exact degeneracy of atomic levels. Direct, for example dipoledipole, interactions make the system different from the collection of independent radiators, the extension of the model still allows an exact solution [55, 56. However, even if not in such a pure form, the super-radiance appears as an important physical effect with abundant applications; the internal states of the radiators are effectively coupled through the common radiation field.

For our goal, the main lesson is that the quantum states, along with possible direct mixing by interactions, can be coupled also through the continuum of open decay channels. Since the continuum coupling determines the width $\Gamma$, or the lifetime $\tau \sim \hbar / \Gamma$, the states become quasistationary and can be characterized by a complex energy,

$$
\mathcal{E}=E-\frac{i}{2} \Gamma .
$$

Similarly to standard perturbation theory, the efficiency of coupling is determined by the ratio of the coupling strength to the energy spacing between the coupled states. If the width $\Gamma$ is of the order of, or exceeds, the spacing between real energies $E$, coupling through the continuum turns out to be effectively strong. This transition to the regime of overlapping resonances supplies the system by the new collectivity - the possibility of coherent decay.

\subsection{Transport through a lattice}

The idea of generalized super-radiance works for many open quantum systems. We illustrate this by a seemingly very different example [13] taken from con- 
densed matter physics, where we will see the similar character of emerging effects. Let us consider the simplest one-dimensional crystal with identical cells numbered $1,2, \ldots, N$. A particle has levels at energy $\epsilon$ in all cells and the hopping amplitude $v$ to adjacent cells. The edge barriers allow the coupling to environment so that the corresponding decay widths are $\gamma_{L}$ and $\gamma_{R}$. The system can be described by an effective non-Hermitian Hamiltonian with matrix elements in the site representation

$$
\mathcal{H}_{n m}=\epsilon \delta_{n m}+v\left(\delta_{m, n+1}+\delta_{m, n-1}\right)-\frac{i}{2}\left(\gamma_{L} \delta_{n 1} \delta_{m 1}+\gamma_{R} \delta_{n N} \delta_{m N}\right)
$$

which describe the decay to outside with the help of complex energies (6) at the edge sites. In the absence of decay, the internal levels would be split by the hopping and form a crystal band of Bloch waves labeled by the discrete wave vectors $q=1, \ldots, N$. The band is characterized by corresponding energies (we set $\epsilon=0$ )

$$
\epsilon_{q}=2 v \cos \varphi_{q}, \quad \varphi_{q}=\frac{\pi}{N+1} q,
$$

and the eigenvectors $|q\rangle$ with components in the site representation

$$
\langle n \mid q\rangle=\sqrt{\frac{2}{N+1}} \sin \left(n \varphi_{q}\right) .
$$

In this basis, the Hamiltonian (7) is transformed to

$$
\mathcal{H}_{q q^{\prime}}=\epsilon_{q} \delta_{q q^{\prime}}-\frac{i}{2} W_{q q^{\prime}},
$$

with the factorized matrix elements of the anti-Hermitian part,

$$
W_{q q^{\prime}}=\frac{2}{N+1} \sin \varphi_{q} \sin \varphi_{q^{\prime}}\left[\gamma_{L}+(-)^{q+q^{\prime}} \gamma_{R}\right] .
$$

As the eigenstates are delocalized, each of them acquires a width; the maximum width appears in the middle of the energy band. The intrinsic hopping interaction is now substituted with the anti-Hermitian interaction through the continuum. The quasistationary states can be found by diagonalizing the effective Hamiltonian (11). The complex energies $\sqrt{6}$ are given by the roots of the secular equation

$$
1+i\left(\gamma_{L}+\gamma_{R}\right) P_{+}(\mathcal{E})+\gamma_{L} \gamma_{R}\left[P_{-}^{2}(\mathcal{E})-P_{+}^{2}(\mathcal{E})\right]=0,
$$

where

$$
P_{ \pm}(\mathcal{E})=\frac{1}{N+1} \sum_{q}( \pm)^{q} \frac{\sin ^{2} \varphi_{q}}{\mathcal{E}-\epsilon_{q}} .
$$

In all cases the total width of all resonances $\sum_{q} \Gamma_{q}$ has to be equal to the trace, $\gamma_{L}+\gamma_{R}$, of $W$. Two possible limiting situations clearly come out. At weak continuum coupling, the individual Bloch states give rise to isolated resonances 
which can still be labeled by the wave vector $q$. Neglecting the last term in eq. (12), we find the widths as the diagonal elements of $W$,

$$
\mathcal{E}_{q} \approx \epsilon_{q}-\frac{i}{2} \Gamma_{q}, \quad \Gamma_{q}=W_{q q},
$$

which, by use of eq. (9), can be presented in an obvious form

$$
\Gamma_{q}=\gamma_{L}|\langle 1 \mid q\rangle|^{2}+\gamma_{R}|\langle N|q|\rangle|^{2} .
$$

The decay here serves as an analyzer singling out the components of the quasistationary state matched to the specific decay channel, left or right in this model. Such arguments are usually used as a justification [57] for identifying the width distribution in a compound nucleus with the distribution of the single components of the compound nuclear wave function. For reduced neutron widths of low-lying neutron resonances with Gaussian distribution of basis components this gives rise to the Porter-Thomas distribution (PTD) [58]. However, this result and underlying logic are valid only in the lowest approximation with respect to weak continuum coupling of non-overlapping unstable states.

In the opposite limiting case of strong continuum coupling, the lifetime with respect to the irreversible decay outside is shorter than for the hopping between the sites, $\gamma_{L, R} \gg v$. In this limit (and $N \gg 1$ ), one can neglect the band splitting $\epsilon_{q}$ in the denominators of $P_{ \pm}$in eq. (13), so that $P_{-}$vanishes while $P_{+} \rightarrow 1 /(2 \mathcal{E})$. Then two roots are shifted along the imaginary energy axis defining

$$
\mathcal{E}_{L, R}=-\frac{i}{2} \gamma_{L, R}
$$

This limit of short-lived collective states is an analog of optical Dicke superradiance. (If $\gamma_{L}$ and $\gamma_{R}$ significantly differ, the system will reveal two superradiant transitions [50]). In distinction to usual collective states in a many-body system, here the collectivization proceeds through the widths of resonances, all Bloch waves radiate coherently being coupled through the decay amplitudes. In the $q$-space, the super-radiant states are in the middle of the spectrum, while in the site representation it is trivial to find that they are located at the edges. Therefore, at strong continuum coupling it might be useful to go to the doorway representation [13] that will be discussed in the corresponding section from a more general viewpoint. In the one-channel case, the transformation of the $S$-matrix to such a doorway form can be made explicitly as was shown long ago [59. In the limit (16), remaining $N-2$ intrinsic states are dark being decoupled from the continuum. It is easy to find the correction to eq. (16), where the long-lived (trapped) states acquire small widths, $\propto\left(v / \gamma_{L, R}\right)^{2}$, through the intermediate coupling to the doorway states directly coupled to the continuum. We can also mention that the disorder in the closed system may even amplify the effects of the super-radiance [52 through the formation of localized states more easily decaying outside. We return to the more detailed discussion of quantum transport in Sec. 8.

The common features of our two examples - optical super-radiance and signal transmission through a chain of radiators - are generic for all problems with 
open quantum systems. We have here a kind of phase transition from independent unstable states to collective radiation (or any process developing with, or through, open continuum channels). A typical control parameter regulating this transformation is the relative strength of continuum coupling with respect to the level spacing in the closed system. Below we introduce the universal formalism adjusted for the consistent description of such situations. 


\section{Open quantum system}

\subsection{Projection formalism}

Following 14, 15, 26 we consider a general quantum system that has both bound and continuum states. The states $|c ; E\rangle$ in the continuum will be labelled by total energy $E$ and the quantum numbers of the channels. The channel, $c$, is characterized by the kind(s) of the fragments in the continuum, their intrinsic states, and all discrete quantum numbers (spins, isospins, parity...). Every channel $c$ opens at its threshold energy $E^{c}$. The whole dynamics in the Hilbert space of the system is governed by the Hermitian Hamiltonian $H=H^{\dagger}$, while the reactions $a \rightarrow b$ between various channels, including the elastic one, $b=a$, can be described by the scattering matrix in the channel space, $S^{b a}(E)$, which is unitary, $S^{\dagger}=S^{-1}$.

Using the stationary quantum mechanics we have to solve the Schrödinger equation for the wave function at real energy,

$$
H \Psi_{E}=E \Psi_{E},
$$

where energy $E$ can belong to the discrete spectrum (and then has to be determined by the solution of the equation) or to the continuum, if some channels are open having their thresholds below given value of $E$. In the coordinate representation, those two cases have different boundary conditions. The threshold positions are also to be determined self-consistently by the Schrödinger equation.

It is always possible to formally subdivide the Hilbert space of the problem into two (or more, see for example [60]) complementary parts using the expansion of the unit operator into arbitrarily selected orthogonal projectors,

$$
\hat{1}=\mathcal{P}+\mathcal{Q}, \quad \mathcal{P} \mathcal{P}=\mathcal{P}, \quad \mathcal{Q} \mathcal{Q}=\mathcal{Q}, \quad \mathcal{P} \mathcal{Q}=\mathcal{Q P}=0
$$

Then the Hamiltonian $H$, as any other operator, contains four classes of matrix elements,

$$
H=H_{\mathcal{Q Q}}+H_{\mathcal{Q P}}+H_{\mathcal{P} \mathcal{Q}}+H_{\mathcal{P} \mathcal{P}}
$$

while the wave function has two parts, $\Psi=\mathcal{P} \Psi+\mathcal{Q} \Psi$, which satisfy the coupled set of equations,

$$
\left[H_{\mathcal{Q} \mathcal{Q}}-E\right] \mathcal{Q} \Psi=-H_{\mathcal{Q P}} \mathcal{P} \Psi, \quad\left[H_{\mathcal{P} \mathcal{P}}-E\right] \mathcal{P} \Psi=-H_{\mathcal{P} \mathcal{Q}} \mathcal{Q} \Psi
$$

Formally eliminating the part $\mathcal{P} \Psi$ of the wave function,

$$
\mathcal{P} \Psi=\left(E-H_{\mathcal{P} \mathcal{P}}\right)^{-1} H_{\mathcal{P} \mathcal{Q}} \mathcal{Q} \Psi
$$

we find the equation projected into the class $\mathcal{Q}$,

$$
\mathcal{H}(E) \mathcal{Q} \Psi=E \mathcal{Q} \Psi
$$

where we have to deal with the effective energy-dependent Hamiltonian

$$
\mathcal{H}(E)=H_{\mathcal{Q Q}}+H_{\mathcal{Q P}} \frac{1}{E-H_{\mathcal{P P}}} H_{\mathcal{P} \mathcal{Q}}
$$


It is useful to stress that the formal results here and below do not require any explicit expressions for the projection operators although it can be done in many ways [15], both in Hilbert space and in configuration space. The flexibility of the approach is its important advantage. Working in the Hilbert space we can avoid problems related to the dependence on the coordinate radius of a channel and explicit matching of the solutions at such a surface [61, 62, 63.

The formal solution (21) has to be complemented with rules of working with the singularities emerging when $H_{\mathcal{P P}}$ has eigenvalues at energy $E$. Although the projection procedure can use any subdivision of space, we will assume that the part $\mathcal{P}$ may contain the continuous spectrum $|c ; E\rangle$, so that the second part of eq. (20) has a solution of the homogeneous equation, and in any channel $c$ the singularity appears at energy above the corresponding threshold $\left(E \geq E^{c}\right)$. The standard rule will be applied that real energy $E$ is to be considered as a limiting value from the upper half of the complex energy plane, $E^{(+)}=E+i 0$. With this rule, those states of space $\mathcal{Q}$ which will turn out to be coupled to open channels in $\mathcal{P}$, will acquire the outgoing waves and become unstable. Then the matrix elements of the effective Hamiltonian 23) can be written more in detail as

$$
\begin{gathered}
\mathcal{H}_{12}=H_{12}+\sum_{c^{\prime} c^{\prime \prime}} \int\left(d \tau^{\prime} d \tau^{\prime \prime}\right) d E^{\prime} d E^{\prime \prime} \\
\times\left\langle 1\left|H_{\mathcal{Q P}}\right| c^{\prime}, \tau^{\prime}, E^{\prime}\right\rangle\left(\frac{1}{E^{(+)}-H_{\mathcal{P} \mathcal{P}}}\right)_{c^{\prime}, \tau^{\prime}, E^{\prime} ; c^{\prime \prime}, \tau^{\prime \prime}, E^{\prime \prime}}\left\langle c^{\prime \prime}, \tau^{\prime \prime}, E^{\prime \prime}\left|H_{\mathcal{P} \mathcal{Q}}\right| 2\right\rangle .
\end{gathered}
$$

Here we use an arbitrary orthonormal basis in the $\mathcal{Q}$-space with the basis states labeled as $|1\rangle,|2\rangle, \ldots$ The differentials $\left(d \tau^{\prime} d \tau^{\prime \prime}\right)$ symbolically denote kinematic variables in the channels $c^{\prime}, c^{\prime \prime}$, apart from explicitly indicated energy.

It is always possible 64] to find the eigenchannel basis in the $\mathcal{P}$-space that brings $H_{\mathcal{P} \mathcal{P}}$ to the diagonal form with eigenvalues $E^{\prime}(c ; \tau)$ and the Hamiltonian (24) simplifies to

$$
\mathcal{H}_{12}=H_{12}+\sum_{c} \int\left(d \tau^{\prime}\right) d E^{\prime}\left\langle 1\left|H_{\mathcal{Q P}}\right| c, \tau^{\prime}, E^{\prime}\right\rangle \frac{1}{E^{(+)}-E^{\prime}\left(c, \tau^{\prime}\right)}\left\langle c, \tau^{\prime}, E^{\prime}\left|H_{\mathcal{P} \mathcal{Q}}\right| 2\right\rangle .
$$

For channels closed at energy $E$, there is no singularity, and the superscript $(+)$ can be omitted. For open channels we use the usual identity valid for real $x$,

$$
\frac{1}{E^{(+)}-x}=\text { P.v. } \frac{1}{E-x}-i \pi \delta(E-x),
$$

where P.v. is a symbol of the principal value. This leads us to the "canonical" form of the effective Hamiltonian,

$$
\mathcal{H}=\widetilde{H}-\frac{i}{2} W
$$

The Hermitian part here is

$$
\widetilde{H}=H+\Delta(E)
$$


where $\Delta(E)$ is an off-shell integral in the sense of the principal value,

$$
\Delta_{12}(E)=\text { P.v. } \sum_{c} \int\left(d \tau^{\prime}\right) d E^{\prime}\left\langle 1\left|H_{\mathcal{Q P}}\right| c, \tau^{\prime}, E^{\prime}\right\rangle \frac{1}{E-E^{\prime}\left(c, \tau^{\prime}\right)}\left\langle c, \tau^{\prime}, E^{\prime}\left|H_{\mathcal{P} \mathcal{Q}}\right| 2\right\rangle,
$$

while the anti-Hermitian part is given by

$$
W_{12}(E)=2 \pi \sum_{c(\text { open })} A_{1}^{c}(E) A_{2}^{c *}(E)
$$

and we introduced the effective amplitudes $A_{1}^{c}(E)$ as matrix elements of $H_{\mathcal{Q P}}$ taken on-shell, $E^{\prime}=E$, with all kinematic factors coming from the integral included into their definition. It is important to notice that the real (dispersive) part $\Delta$ of the effective Hamiltonian, sometimes called the energy shift, includes the sum over all channels, open and closed, while the imaginary (absorptive) part $W$ contains only contributions of the channels open at given energy. The kinematic factors (the density of states in the continuum) guarantee that any amplitude $A_{1}^{c}(E)$ vanishes at the threshold energy $E^{c}$. By construction, these amplitudes are combined into a factorized structure that is a consequence (and a mathematical reason) of the unitarity of the scattering matrix [27]. We have already seen this factorization in a specific simple model, eq. (11).

\subsection{Effective Hamiltonian and reaction theory}

The effective Hamiltonian (27) allows us to consider the properties of an open system in a very general fashion. The advantage of this formulation is in the discretization of the problem since we can assume that the internal part of the Hamiltonian, $H_{\mathcal{Q} \mathcal{Q}}$, by construction of our subdivision $(19)$ has only a discrete spectrum, so that the resonances can appear only due to the continuum coupling, $H_{\mathcal{P} \mathcal{Q}}$ and $H_{\mathcal{Q P}}$. This "natural" discretization should not be confused with the discretization of the continuum used frequently in the numerical studies of various reactions [65]; here we are still working within the exact formulation of the theory but the whole dynamics is projected onto the "intrinsic" part of the total Hilbert space.

For a system with $N$ as a (usually very large) dimension of the intrinsic space and $M$ open channels, the amplitudes $A_{1}^{c}$ can be considered as rectangular matrices $N \times M$ which we can label $\mathbf{A}$, so that the matrix of the operator $W$, eq. (30), can be presented as a product

$$
W=2 \pi \mathbf{A} \mathbf{A}^{\dagger} .
$$

In a time-reversal invariant system, the amplitudes $A_{1}^{c}$ can be taken as real numbers; then $\mathbf{A}^{\dagger}=\mathbf{A}^{T}$. This factorized structure facilitates the task of establishing the relation of this approach to the formal theory of reactions [66, 67. On the other hand, this is a mathematical key to super-radiance.

We can introduce the propagator at complex energy $\mathcal{E}$ for the effective Hamiltonian $\mathcal{H}$ as

$$
\mathcal{G}(\mathcal{E})=\frac{1}{\mathcal{E}-\mathcal{H}},
$$


where physical energy axis is a limit $\mathcal{E} \rightarrow E^{(+)}$. The analog of the Dyson equation that connects $\mathcal{G}$ with the propagator $G$ for a closed system,

$$
G(\mathcal{E})=\frac{1}{\mathcal{E}-\tilde{H}},
$$

can be derived algebraically with the help of the factorized structure of $W$ :

$$
\mathcal{G}(\mathcal{E})=G(\mathcal{E})-\frac{i}{2} G(\mathcal{E}) \mathbf{A} \frac{1}{1+(i / 2) \hat{K}(\mathcal{E})} \mathbf{A}^{\dagger} G(\mathcal{E}) .
$$

Here and later the hats mark $M \times M$ matrices in the channel space; in particular, the matrix

$$
\hat{K}(\mathcal{E})=\mathbf{A}^{\dagger} G(\mathcal{E}) \mathbf{A}
$$

describes the full propagation inside the system from the entrance channel to the exit channel. The series of such propagations summed over in the operator $[1+(i / 2) \hat{K}]^{-1}$ of eq. 34$)$ corresponds to the full process of the reaction through the system by an infinite number of intermediate excursions from the intrinsic space to the continuum. The matrix (35) substitutes in this theory the standard $R$-matrix [21, 23, 63].

The part of the amplitude for the reaction $b \rightarrow a$ that proceeds through the states of the subspace $\mathcal{Q}$ is now an element $\left(T_{\mathcal{Q}}\right)^{a b}$ of the $M \times M$ matrix

$$
\hat{T}_{\mathcal{Q}}(\mathcal{E})=\mathbf{A}^{\dagger} \mathcal{G}(\mathcal{E}) \mathbf{A}=\hat{K}(\mathcal{E}) \frac{1}{1+(i / 2) \hat{K}(\mathcal{E})} .
$$

The total reaction amplitude includes also the direct processes determined by the dynamics in the $\mathcal{P}$-space without entering the intrinsic state,

$$
\hat{T}=\hat{T}_{\mathcal{P}}+\hat{T}_{\mathcal{Q}},
$$

where $\mathcal{E} \rightarrow E^{(+)}$. The item $\hat{T}_{\mathcal{P}}$ is responsible for what is called potential scattering. The complete scattering matrix can be written as

$$
\hat{S}(\mathcal{E})=1-i \hat{T}(\mathcal{E})=-i \hat{T}_{\mathcal{P}}+\frac{1-(i / 2) \hat{K}(\mathcal{E})}{1+(i / 2) \hat{K}(\mathcal{E})}
$$

and the cross sections in open channels are defined by the corresponding matrix elements of the $\hat{S}$-matrix at physical energy, with the interference of direct and resonance scattering taken into account. The amplitudes $\mathbf{A}$ also contain phases of the potential scattering at the entrance and exit of the channels. The resonance part, closely related to the intrinsic structure of the system, will capture our main attention. In the absence of direct processes, this part is evidently unitary by itself.

The amplitudes $T_{\mathcal{Q}}^{a b}(\mathcal{E})$, according to the first equality (36), have the poles in the complex plane of energy $\mathcal{E}$ coming from the poles of $\mathcal{G}(\mathcal{E})$, which are the eigenvalues (6) of the effective Hamiltonian $\mathcal{H}$. Those eigenvalues determine the "compound-nucleus" resonance spectrum of the system. We use here this term 
only to express the fact that the wave function of such a state has components in the $\mathcal{Q}$-space. Whether a resonance indeed agrees with the notion of compound nucleus in the standard meaning of this concept can be decided by the comparison of the resonance lifetime $\tau \sim \hbar / \Gamma$ with the characteristic equilibration time inside the system. As stated by Feshbach [15], "the effective Hamiltonian method is most convenient for nuclear reactions in that it differentiates ... between compound and direct nuclear reactions". On the real energy axis, the amplitudes have branch points at the channel thresholds.

\subsection{Emergence of resonances}

We start with a simple example of a resonance that emerges as a result of interaction between the two subspaces (18). This will illustrate a direct relation between the method under discussion and the textbook scattering theory. The formalism, apparently first used by Fano [16] in application to the autoionizing states of an atom, immediately follows from the general projection method. In fact, this approach is similar to the consideration of the spreading width of a collective ("bright") state interacting with the background of secondary states and in such a form it was suggested by Rice [68. This analogy will reappear in the discussion of the doorway states in Sec. 6 .

If we consider only a small number of special intrinsic states, it is convenient to project the entire dynamics into the external $\mathcal{P}$-space. Eliminating the part $\mathcal{Q} \Psi$ of the wave function, we come to the equation

$$
\left[H_{\mathcal{P} \mathcal{P}}-E\right] \mathcal{P} \Psi=H_{\mathcal{P} \mathcal{Q}} \frac{1}{H_{\mathcal{Q} \mathcal{Q}}-E} H_{\mathcal{Q P}} \mathcal{P} \Psi .
$$

Here energy $E$ belongs to the continuous spectrum, and the homogeneous equation, $\left[H_{\mathcal{P} \mathcal{P}}-E^{\prime}\right] \mathcal{P} \Psi=0$, has solutions $\psi_{E^{\prime}}^{c}$ corresponding to the asymptotic channels $c$ included in the $\mathcal{P}$-space. The right hand side of eq. $(39)$ describes the intrinsic states with the same conserved quantum numbers which are coupled to the continuum and, if above thresholds, can reveal autoionization. As a demonstration of principles, we consider here only the case of one intrinsic state $\Phi$ with unperturbed energy $H_{\mathcal{Q} \mathcal{Q}}=\epsilon$ coupled to a single channel [if not all open channels are included into the $\mathcal{P}$-space, we have to consider the limit $E \rightarrow E^{(+)}$in the denominator of the second term in eq. [39]].

The solution of eq. 177 is a superposition

$$
\Psi_{E}=a(E) \Phi+\int d E^{\prime} b\left(E, E^{\prime}\right) \psi_{E^{\prime}}
$$

a direct analog of the spreading of a bright state in the discrete spectrum. Omitting from our notations the dependence of $a$ and $b$ on running energy $E$, we obtain the set of coupled equations for the amplitudes $a$ and $b\left(E^{\prime}\right)$ in terms of the continuum coupling amplitudes $A\left(E^{\prime}\right)=\left\langle\Phi\left|H_{\mathcal{Q P}}\right| \psi_{E^{\prime}}\right\rangle$, as in eq. 330,

$$
(E-\epsilon) a=\int d E^{\prime} A\left(E^{\prime}\right) b\left(E^{\prime}\right), \quad\left(E-E^{\prime}\right) b\left(E^{\prime}\right)=A^{*}\left(E^{\prime}\right) a .
$$


The existence of the homogeneous solution in the continuum at $E^{\prime}=E$ leads to the general result in the form of the principal value plus the delta-function,

$$
b\left(E^{\prime}\right)=\left[\mathrm{P} \cdot \mathrm{v} \cdot \frac{1}{E-E^{\prime}}+r(E) \delta\left(E-E^{\prime}\right)\right] A^{*}\left(E^{\prime}\right) a,
$$

where the residue factor $r(E)$ is real and has to be determined self-consistently, while in the analogous situation in eq. (25) we could simply use the standard identity (26). The first equation of (41) determines now

$$
r(E)=\frac{E-\epsilon-\Delta(E)}{|A(E)|^{2}},
$$

where $\Delta(E)$ is the simplified form of the principal value integral 29$)$. Introducing the coordinate representation of the scattering problem, one can show [16] that this residue defines the phase shift $\delta$,

$$
\tan \delta(E)==-\frac{\pi}{r(E)} .
$$

Finally, the normalization of the full wave function by the delta-function of energies in the continuum, allows one to find the remaining amplitude

$$
|a(E)|^{2}=\frac{|A(E)|^{2}}{[E-\epsilon-\Delta(E)]^{2}+\Gamma^{2} / 4},
$$

of resonance shape with the energy shift $\Delta(E)$ and the width

$$
\Gamma(E)=2 \pi|A(E)|^{2},
$$

in agreement with the definition of eq. (30). Both, the energy shift and the width, are energy-dependent. The shift $\Delta(E)$ vanishes if one can neglect the energy variation of the amplitudes $A(E)$. The physical characteristics changing rapidly as a function of energy near the resonance peak lead to the highly asymmetric curves for cross sections which is a well known feature of autoionization; the typical example of the $2 s 2 p$ excited state in the helium atom was analyzed in detail in the original work [16].

\subsection{Overlapping resonances and super-radiance}

Our next step is the analysis of the eigenvalues and the eigenfunctions of the effective non-Hermitian Hamiltonian $\mathcal{H}$. To emphasize the physics of superradiance and related transformations of the resonance spectrum, we neglect for a while the energy dependence of the amplitudes $A_{1}^{c}$. This assumption will be lifted in the continuum shell model when the threshold dependence of the amplitudes will be important for the spectroscopy of the resonances. If our energy is not close to thresholds, the energy dependence is smooth. In the case of many resonances with the same quantum numbers, such as the lowenergy neutron resonances in heavy nuclei, the energy dependence is common 
for all of them and can be usually subtracted [57] using the so-called reduced widths. Special precaution should be taken if the compound resonances come from the single-particle resonance that served as a doorway state on the way to a compound nucleus [69. In this case the shape of the doorway strength function may distort the reduced widths (we return to the width distribution in Sec. 7.2).

The two parts of the effective Hamiltonian (27) do not commute in general and cannot be brought simultaneously to a diagonal form by an orthogonal transformation of the basis. The complex diagonalization of $\mathcal{H}$ leads to a biorthogonal set $\left\{\chi_{r}, \chi_{r}^{*}\right\}$ of right and left eigenfunctions which have complex conjugate eigenvalues $\mathcal{E}_{r}$ and $\mathcal{E}_{r}^{*}$. All eigenvalues are located in the lower part of the complex energy plane; the resonance widths $\Gamma_{r}$, eq. (6), are positive. This can be easily seen from the properties of the effective Hamiltonian (27). Its eigenvalue equation reads

$$
\left[\mathcal{E}_{r}-H_{\mathcal{Q} \mathcal{Q}}-H_{\mathcal{Q P}} \frac{1}{E^{(+)}-H_{\mathcal{P P}}} H_{\mathcal{P} \mathcal{Q}}\right] \chi_{r}=0 .
$$

Taking the matrix element with the biorthogonal partner $\chi_{r}^{*}$, we obtain

$$
\mathcal{E}_{r}-\mathcal{E}_{r}^{*}=\left\langle\chi_{r}^{*}\left|H_{\mathcal{Q P}}\left(\frac{1}{E^{(+)}-H_{\mathcal{P P}}}-\frac{1}{E^{(-)}-H_{\mathcal{P P}}}\right) H_{\mathcal{P} \mathcal{Q}}\right| \chi_{r}\right\rangle .
$$

The principal value terms cancel, and the imaginary part of the eigenenergy determines the positively defined width (the so-called Bell-Steinberger relation):

$$
\Gamma_{r}=2 \pi\left\langle\chi_{r}^{*}\left|H_{\mathcal{Q} \mathcal{P}} \delta\left(E-H_{\mathcal{P} \mathcal{P}}\right) H_{\mathcal{P} \mathcal{Q}}\right| \chi_{r}\right\rangle .
$$

It might be convenient to work instead with a complete orthogonal basis of one of the parts, $\widetilde{H}$ or $W$, which will be distinguished as the internal and doorway representation, respectively. Let $|\alpha\rangle$ be the eigenfunctions of $\widetilde{H}$ with real eigenvalues [as the Bloch waves in the case of the lattice, eqs. (8) and (9)],

$$
\widetilde{H}|\alpha\rangle=\epsilon_{\alpha}|\alpha\rangle .
$$

The part $W$ of the total Hamiltonian remains factorized after the unitary transformation to the basis $\alpha\rangle$, compare eq. (11),

$$
W_{\alpha \beta}=2 \pi \sum_{1,2 ; c}\langle\alpha \mid 1\rangle A_{1}^{c} A_{2}^{c *}\langle 2 \mid \beta\rangle \equiv 2 \pi \sum_{c} B_{\alpha}^{c} B_{\beta}^{c *} .
$$

In the internal representation, we deal with the Hamiltonian

$$
\mathcal{H}_{\alpha \beta}=\epsilon_{\alpha} \delta_{\alpha \beta}-\frac{i}{2} 2 \pi \sum_{c(\text { open })} B_{\alpha}^{c} B_{\beta}^{c *} .
$$

According to eq. (34), the complex eigenvalues are the roots of

$$
\operatorname{Det}\left\{1+\frac{i}{2} \hat{K}(\mathcal{E})\right\}=0 \text {. }
$$


Two opposite limiting regimes naturally come into consideration. At weak coupling to continuum, the anti-Hermitian part is a perturbation to a standard intrinsic Hamiltonian of the shell-model type. Then we expect the appearance of narrow resonances corresponding to long-lived states barely coupled to decay channels. At strong coupling, the main role is played by the part $W$. In general, the whole picture including the resonances depends on the running energy $E$ of the system, although this might be not important far from thresholds.

Let us make simple estimates assuming that the intrinsic states $|\alpha\rangle$ are approximately of the same degree of complexity and have a similar strength of continuum coupling so that it makes sense to use the average parameters. The transformation (51) preserves the trace of $W$ which we denote $w$. This trace will be the same after the final complex transformation to the eigenbasis of $\mathcal{H}$, and the typical width of a resonance in the case of weak coupling will be $\Gamma \sim w / N$. If this width is small compared to the spacings $D$ between the levels $\epsilon_{\alpha}$, the usual perturbation theory should be valid. This can be expressed with the aid of the coupling parameter $\kappa \sim(\Gamma / D) \ll 1$, or, in global characteristics, $w /(N D) \ll 1$, the total summed width is small compared to the energy interval covered by the relevant intrinsic levels. Only the diagonal part of $W$ in the representation (52) is important in this limit, and the resonance states, as in the example [15), still can be labeled by the quantum numbers of $\widetilde{H}$, eq. 50 ,

$$
\mathcal{E}_{r} \Rightarrow \mathcal{E}_{\alpha}=\epsilon_{\alpha}-\frac{i}{2} \Gamma_{\alpha}, \quad \Gamma_{\alpha}=2 \pi \sum_{c}\left|B_{\alpha}^{c}\right|^{2} .
$$

In this regime the reactions reveal narrow isolated resonances $|\alpha\rangle$ with partial widths

$$
\gamma_{\alpha}^{c}=2 \pi\left|B_{\alpha}^{c}\right|^{2}, \quad \Gamma_{\alpha}=\sum_{c} \gamma_{\alpha}^{c}
$$

This is, for example, the typical picture for low-energy neutron resonances.

The situation drastically changes when we approach the strong coupling regime, $\kappa \sim 1$. Then $w \sim N D$, the resonances start to overlap and the situation of similar isolated resonances turns out to be unstable with respect to the sharp redistribution of widths. To illustrate this point we start with the one-channel case, when eq. (53) reads

$$
1+\frac{i}{2} \sum_{\alpha} \frac{\gamma_{\alpha}}{\mathcal{E}-\epsilon_{\alpha}}=0
$$

In the same basis we now can see a very different solution in the limit of $\kappa \gg 1$, namely an emergence of a very broad state that accumulates almost the whole summed width $w=\sum_{\alpha} \gamma_{\alpha}$. If $w \gg N D$, all intrinsic states would be covered by the total width, so that introducing the centroid of real energies weighted with their original widths $\gamma_{\alpha}$,

$$
\bar{\epsilon}=\sum_{\alpha} \epsilon_{\alpha} \frac{\gamma_{\alpha}}{w}
$$


we immediately find from eq. (56) the broad pole $\mathcal{E}_{1}=E_{1}-(i / 2) \Gamma_{1}$ with $\mathcal{E}_{1}$ close to the centroid (57) and the width $\Gamma_{1}$ close to $w$. The remaining $N-1$ states are in this limit very narrow (trapped). With corrections of the second order in $1 / \kappa \ll 1$, this special root of eq. (56) is given by

$$
\begin{gathered}
E_{1}=\bar{\epsilon}+4 \sum_{\alpha} \epsilon_{\alpha} \frac{\left(\epsilon_{\alpha}-\bar{\epsilon}\right)^{2} \gamma_{\alpha}}{w^{3}}, \\
\Gamma_{1}=w-4 \sum_{\alpha} \frac{\gamma_{\alpha}\left(\epsilon_{\alpha}-\bar{\epsilon}\right)^{2}}{w^{2}} .
\end{gathered}
$$

The trapped states have narrow widths $\Gamma_{t} \sim w /\left(N \kappa^{2}\right)$ which are much smaller than the average starting width $\gamma \sim w / N$. The more detailed analytical solutions can be derived in a model [35] with equidistant intrinsic spectrum and weakly fluctuating $\gamma_{\alpha}$.

This example shows a generic mechanism of the formation of the superradiant state. When the widths of individual resonances overlap and $\kappa>1$, the dominating interaction between the resonances proceeds through the common channels in the continuum. Roughly speaking, energy uncertainty of an unstable state makes a virtual decay of this state possibly end with the return in the $\mathcal{Q}$ space to a neighboring overlapped state. This coupling leads to the resonance collectivization and the segregation of a super-radiant combination, the story one can identify in the two examples discussed in Sec. 2. It is easy to see that the super-radiant state $\left|\Phi_{1}\right\rangle$ is a superposition of intrinsic states best adjusted to the decay channel. Expressed as a vector in $\mathcal{Q}$-space, it is nearly aligned along the decay amplitude vector $\mathbf{B}=\left\{B_{\alpha}\right\}$, its transverse components are small, $\sim 1 / \kappa$. In the example of a periodic lattice of Sec. 2 , this corresponded to the localization of the super-radiant states at the spatial edges of the chain opened for the decay. The trapped states return to the non-overlap regime and belong mainly to the subspace orthogonal to $\mathbf{B}$.

We can also establish the hierarchy of time intervals corresponding to various parts of the dynamics involved in this transition. At $\kappa \gg 1$, the longest time is the lifetime of the trapped states, $\tau_{t} \sim \hbar / \Gamma_{t} \sim \kappa(\hbar / D) \gg \hbar / D$, where the Weisskopf time $\tau_{W} \sim \hbar / D$ [70] describes the recurrence of the intrinsic wave packet in its evolution in the closed space without decays. The inequality $\tau_{t}>\tau_{W}$ means that the wave functions of trapped states can be considered fully equilibrated as corresponds to the proper compound stage. The next time $\hbar / N D$ is even shorter than the recurrence time representing essentially the lifetime of a certain intrinsic basis state $|1\rangle$ with respect to the intrinsic spreading. Finally, the lifetime of the super-radiant state is the shortest, $\tau_{s} \sim \hbar / \Gamma_{1} \sim(\hbar / N D \kappa)$.

Going to the case of two open channels, $a$ and $b$, we will have two $N$ dimensional amplitude vectors, $\mathbf{B}^{a}$ and $\mathbf{B}^{b}$. In accordance with that, in the regime of strong continuum coupling we find two broad states, $\left|\Phi_{ \pm}\right\rangle$, which divide among themselves the lion's share of the total width $w$. Their complex energies, $\mathcal{E}_{ \pm}=E_{ \pm}-(i / 2) \Gamma_{ \pm}$depend on the amplitudes, $\gamma^{a, b}=2 \pi\left|\mathbf{B}^{a, b}\right|^{2}$, of 
the vectors $\mathbf{B}$ and the angle $\vartheta$ between them [13]. In particular, after the superradiant transition, $\kappa \gg 1$,

$$
\Gamma_{ \pm} \approx \frac{1}{2}\left[w \pm \sqrt{w^{2}-4 \gamma^{a} \gamma^{b} \sin ^{2} \vartheta}\right] .
$$

If the decay amplitudes are close to orthogonality, the channels become effectively decoupled and we obtain two super-radiant states (the left and right edge states in the lattice example). In the "parallel" situation, $\vartheta \rightarrow 0$, the state $\left|\Phi_{-}\right\rangle$with destructive interference of the two decay channels joins the set of trapped states, while the constructive interference leaves only one super-radiant state $\left|\Phi_{+}\right\rangle$with $\Gamma_{+} \rightarrow w$. The long-lived $N-2$ states are concentrated in the subspace orthogonal to that spanned by the vectors $\mathbf{B}^{a, b}$.

We can qualitatively anticipate the results for a multi-channel case. The matrix $W$ has a rank $M$ equal to a number of open channels. This defines the number of its non-zero eigenvalues; for $M=1$, the only nonzero eigenvalue of this matrix equals its trace $w$. Therefore, after the transition to the strong continuum coupling, the natural basis would be that of the doorway representation, where $M$ eigenvectors of $W$ with non-vanishing eigenvalues are used along with $N-M$ vectors from the complementary subspace. Here we expect the appearance of $M$ broad states sharing the total width, while $N-M$ states are getting trapped. Such a picture was clearly observed in the numerical calculations [8] of nuclear structure of ${ }^{16} \mathrm{O}$ in the region of the dipole resonance studied as a function of the continuum coupling strength. However, if the multidimensional angles $\vartheta^{a b}$ between the amplitude vectors fluctuate, the possible destructive interference can suppress the manifestations of super-radiance, especially in the situation of many open channels.

The doorway physics will be discussed in more detail later. Here we just mention one simple but instructive model [52, a set of $n$ single-fermion levels $\epsilon_{j}$, including the upper one, $j=1$, in the continuum with complex energy $\epsilon_{1}-$ $(i / 2) \gamma$. In the system of $k<n$ fermions, the interaction mixes the configurations partially populating the level $\epsilon_{1}$ and opening the decay for other states. If all $n ! /[k !(n-k) !]$ many-body states have energy higher than the decay threshold, in the presence of the interaction they acquire the widths and shift down from the real energy axis into the complex energy plane. However, with the increase of the width $\gamma$, complex plane trajectories of some states turn back and again approach the vicinity of the real axis while the rest of states have monotonously growing widths, Fig. 1. The number of the latter (super-radiant) is $(n-$ $1) ! /[(k-1) !(n-k) !]$, the number of configurations of remaining $(k-1)$ particles compatible with the occupied doorway state $\mid 1$ ) (these particles share the orbits $j \neq 1$ ).

The segregation of super-radiant and trapped states occurs in fact rather sharply when the parameter $\kappa$ reaches the value close to 1 . This is illustrated by Fig. 2 taken from Ref. [12] where the system of 1000 intrinsic states coupled uniformly to 250 channels was modeled. The random distribution of the widths at weak coupling evolves releasing the short-lived states until the 
cloud of super-radiant states sharply separates from the rest of the system that contains trapped states with prolonged lifetimes. 


\section{Nuclear continuum shell model}

As mentioned in the previous Section, the story of the effective non-Hermitian Hamiltonian was essentially started with the search for an appropriate theory for description of resonance nuclear reactions. Although we will argue that the theory is much more universal and will show the examples of its broad applications, still nuclear physics provides probably the most explored arena for using this approach. At least partly, this can be explained not only by historical reasons. The main trends of the development of modern nuclear science clearly lead to the problems, where the continuum effects are exceedingly important.

Experimental nuclear physics shifted its center of interest to the nuclei far from the valley of stability. The new physical problems combine here the questions of limits of nuclear existence with the astrophysical mysteries of the origin of chemical evolution and supernova explosion. The standard shell model is constructed to work in the discrete spectrum of nuclear, atomic or molecular states. The beta-unstable nuclei close to the drip lines are loosely bound, in many cases only the low-lying states (or just the ground state) are particle-stable. Therefore the conventional distinction between structure and reactions (the division between the subjects of the research as well as between the researchers) becomes obsolete and it is necessary to find ways to overcome this barrier. In fact, a similar situation exists for the entire class of open or marginally stable mesoscopic systems, including various micro- and nano-scale channels for transmission of quantum information.

In loosely bound nuclei any real or virtual excitation is related to the continuum part of the energy spectrum. The sharp discrimination between nuclear structure and nuclear reaction disappears and our theoretical description should cover both regions. In the classical problem of neutron resonances in heavy nuclei we also are working in the continuum very close to the threshold of the discrete spectrum. But there (we return later to this class of problems) the details of the underlying structure are less important since the long-lived resonances correspond to chaotic states of the compound nucleus, where one can use statistical approaches justified by random matrix theory. In nuclei close to the drip lines we are interested in details of weakly bound structure and in the spectroscopy of individual resonances. Therefore we need the generalization of the shell model that would allow the full understanding of this region and unified description of structure and reactions.

Here the approach based on the effective non-Hermitian Hamiltonian becomes extremely appropriate (along with other kindred methods, like for example the Gamow shell model [32, 33]). The signatures of super-radiance were seen (however not fully understood) in numerical simulations by Moldauer [71. The first clear indication can be found in Ref. [8, where the dipole excited states in ${ }^{16} \mathrm{O}$ were considered in a simplified shell model version, with decay probabilities (level widths) taken as variable parameters. The results sharply changed with the degree of openness of the system. Depending on the number $M$ of open

channels, as the escape widths were increased, the formation of $M$ very broad resonances was observed, with other states becoming more and more narrow. 
The analogy to optical super-radiance and the explanation of these effects were suggested in Refs. 9, 10; the first results of applications to the nuclear shell model were given in the review article by Rotter [28. Our discussion will be mainly based on the later development [72, 29, 30, 31].

\subsection{Two-level example}

We start with the illustration of dynamics on the borderline between discrete spectrum and continuum. We assume that the intrinsic, $\mathcal{Q}$, space contains only two relevant states coupled to the single open decay channel through real amplitudes $A_{1,2}$. This simple example [29] in fact has many non-trivial physical applications $72,73,74,75,76,77$ including experimental studies with microwave cavities [78. It might serve [79] as the starting model for such a nucleus as ${ }_{3}^{11} \mathrm{Li}_{8}$, where the open channel contains the residual nucleus ${ }^{9} \mathrm{Li}$ and emitted neutron pair. For the pair we assume only one state ${ }^{1} S_{0}$ of relative motion. In some sense, this is an original Cooper effect [80] where one pair can be bound on the background of other fermions even if the primary attractive interaction within the pair is insufficient for binding; the attraction in the vicinity of the nucleus is also helped by the exchange of the collective excitations of the core [81, 82.

The most energetically favorable wave function of the Cooper pair is a combination of available for the pair orbitals, for example $0 p_{1 / 2}$ and $1 s_{1 / 2}$ in our simplified model (in reality, probably, it has more components). Both singleparticle states are in the continuum since the adjacent isotope ${ }^{10} \mathrm{Li}$ is unbound. The effective Hamiltonian includes interactions of both types, the usual Hermitian $v$, such as pairing, and the anti-Hermitian, through the decay channel, and it can be written in a general form (27),

$$
\mathcal{H}=\left(\begin{array}{cc}
\epsilon_{1}-(i / 2) A_{1}^{2} & v-(i / 2) A_{1} A_{2} \\
v-(i / 2) A_{1} A_{2} & \epsilon_{2}-(i / 2) A_{2}^{2}
\end{array}\right)
$$

where we assume that the real energies $\epsilon_{1,2}$ are both positive (above threshold that is set at zero energy), and the factor $\sqrt{2 \pi}$ is included into the amplitudes $A_{1,2}$.

The complex eigenvalues of this Hamiltonian are formally given by

$$
\begin{gathered}
\mathcal{E}_{ \pm}=\frac{\epsilon_{1}+\epsilon_{2}-(i / 2)\left(\gamma_{1}+\gamma_{2}\right)}{2} \\
\pm \frac{1}{2} \sqrt{\left(\epsilon_{1}-\epsilon_{2}\right)^{2}+4 v^{2}-\frac{\left(\gamma_{1}+\gamma_{2}\right)^{2}}{4}-i\left[\left(\epsilon_{1}-\epsilon_{2}\right)\left(\gamma_{1}-\gamma_{2}\right)+4 v A_{1} A_{2}\right]}
\end{gathered}
$$

where $\gamma_{1,2}=A_{1,2}^{2}$. This result is only formal if, as it happens in the realistic shell model, the amplitudes $A_{1,2}$ are energy dependent and have to vanish at threshold. A typical complication in such problems is that the location of threshold is unknown a-priori. A consistent solution would require to consider simultaneously the daughter nucleus and maybe even the chain of decays as we will show later. 
The behavior of complex energies predicted by eq. 62 is non-trivial 29 , 73. The Hermitian perturbation $v$ leads to the standard level repulsion; in the absence of open channels, the lower root $E_{-}$is pushed down crossing the zero level at $v^{2}=\epsilon_{1} \epsilon_{2}$ and becoming bound. The intrinsic states are mixed and, if their widths were very different, after the mixing they become more uniform, the widths are attracted. Contrary to that, the imaginary perturbation in the second order makes the levels to attract each other, while the widths are repelled, and this is the beginning of the road to super-radiation. As this process is determined by the ratio of widths to the level spacing, it occurs immediately for degenerate levels, as in the ideal Dicke model. Indeed, for $\epsilon_{1}=\epsilon_{2} \equiv \epsilon$ and $v=0$, we obtain

$$
E_{+}=\epsilon, \quad E_{-}=\epsilon-\frac{i}{2}\left(\gamma_{1}+\gamma_{2}\right),
$$

the super-radiant state absorbs the whole width while the remaining state is stable although its energy is embedded into continuum. In the same way, for any number of degenerate intrinsic levels, the real Hamiltonian $\widetilde{H}$ is given by the unit matrix, and the energy splitting is fully defined by $W$ that produces $M$ non-zero eigenvalues. Another new effect is the possibility of crossing for the complex poles [83], analogous to the well known behavior of two coupled classical damped oscillators 84 .

The non-universal dependence of amplitudes $A_{1,2}$ [and even of real energies $\epsilon_{1,2}$ if the principal value part $\Delta(E)$, eq. $[29)$, is important] on running energy $E$ makes the analysis much more involved [13, 29, 31, since the complex poles move as a function of energy, and the position $E$ of a resonance on the real energy axis is defined by the solution of $\operatorname{Re} \mathcal{E}(E)=E$. The two levels in our problem are then found by diagonalizing different matrices. One should carefully take care of the vanishing width at threshold, so for example, when the lower level becomes bound, $E_{-}=\Gamma_{-}=0$. In the model of ${ }^{11} \mathrm{Li}$, one can assume the usual energy dependence from the potential model for $p$ - and $s$-orbitals,

$$
\gamma_{p}(E)=\beta E^{3 / 2}, \quad \gamma_{s}(E)=\alpha \sqrt{E} .
$$

In this way we obtain the self-consistent solution when, simultaneously with $v^{2}=\epsilon_{p} \epsilon_{s}$, we come to $E_{-}=\Gamma_{-}=0$. For special conspiracy of the parameters, the system also reveals a bound state embedded in the continuum. In general, bound states, resonances and energy dependence of the amplitudes are strongly correlated.

The same effective Hamiltonian determines the resonance part of the scattering amplitude,

$$
T(E)=\frac{E\left(\gamma_{1}+\gamma_{2}\right)-\gamma_{1} \epsilon_{2}-\gamma_{2} \epsilon_{1}+v A_{1} A_{2}}{\left(E-\mathcal{E}_{-}\right)\left(E-\mathcal{E}_{+}\right)},
$$

where we correct a misprint in the corresponding equation of [29]. Only in the degenerate case of eq. (63), we see a clear Breit-Wigner resonance on the super-radiant state,

$$
T(E)=\frac{\gamma_{1}+\gamma_{2}}{E-\epsilon+(i / 2)\left(\gamma_{1}+\gamma_{2}\right)} .
$$


The dark state with $\Gamma=0$ is not seen in the scattering. Fig. 3 taken from [29] shows the typical cross sections for the positive, $3 \mathrm{a}$, and negative, $3 \mathrm{~b}$, signs of the product $A_{1} A_{2}$ at certain values of parameters. The peak at low energy in the case of the positive product and $v^{2}>\epsilon_{1} \epsilon_{2}$, when the lower level is loosely bound, demonstrates a typical example of the threshold maximum of sub-resonant nature, whereas the remaining broad upper resonance at higher energy is not shown. For the negative product $A_{1} A_{2}$, the presence of the threshold singularity is responsible for the finite value of the cross section at zero energy.

\subsection{Realistic calculations}

In all realistic applications, the truncation of both, $\mathcal{P}$ and $\mathcal{Q}$, spaces is inevitable. With a truncated set of single-particle orbitals, the space of many-body states in the nuclear shell model, built, with full antisymmetrization required by Fermistatistics, on these orbitals, can be naturally identified with our $\mathcal{Q}$-space. The configuration interaction within this space is given by a set of matrix elements, traditionally of two-body but in modern versions also of three-body character [85, 86. The solution process is usually based on a large-scale diagonalization of the Hermitian Hamiltonian matrix. The single-particle states can be conveniently defined in a spherically symmetric basis being labeled by the spin-spatial quantum numbers $(n, \ell, j, m)$ and the isospin projection $\tau$. The diagonalization then leads to the many-body states with good quantum numbers of total spin and parity. The deformed mean field and corresponding rotational bands can emerge from the solution if the original space is sufficiently large.

In this spherical shell model, the interaction matrix elements are defined for any pair of initial and final two- or three-body states with the same constants of motion - total spin quantum numbers $(J, M)$, parity, and isospin if there are no forces breaking charge symmetry. The number of such allowed matrix elements can be large but still by orders of magnitude smaller than the dimension of the $\mathcal{Q}$-space. The values of the matrix elements can be in principle theoretically derived from the vacuum nucleon and meson interactions fitted by few-body observables. In practice, these values have to be phenomenologically adjusted in order to reproduce thousands of experimental data. Here the theory has already a rich experience and many successes. Unfortunately, such an experience is essentially absent for the matrix elements in the $H_{\mathcal{P} \mathcal{Q}}+H_{\mathcal{Q P}}$ class for coupling of the intrinsic space with the outside world.

In the first stage of the development, usually a potential of the WoodsSaxon type and simple short-range residual forces were used in all parts of the effective Hamiltonian; the open channels were restricted to the one particle in the continuum $[87,88$. The single-particle resonances emerge from the internal shell-model bound states embedded in the open continuum. In a similar way, but with a more advanced model of intrinsic dynamics, it was possible to describe the inelastic electron scattering with the knock-out of a nucleon [89].

Since the effective Hamiltonian is energy-dependent, in general the position $E_{r}(E)$ and the width $\Gamma_{r}(E)$ of a resonance are parameters of the eigenvalues (6) of $\mathcal{H}(E)$ which depend on running energy of the experiment. The strictly 
formulated theory is capable of predicting directly the observable cross section $\sigma^{a b}(E)$ for given entrance and exit channels. Then the convenient working definitions of the resonance parameters are the roots of obvious equations, $E_{r}=$ $E_{r}\left(E=E_{r}\right)$ and $\Gamma_{r}=\Gamma_{r}\left(E=E_{r}\right)$. The shape of the resonance curve is evolving with the energy of the experiment being in general non-symmetric with respect to the position $E_{r}$ [87] and reveals a cusp required by unitarity at the threshold for opening a new channel $90,91,92$. The cusps are well known experimentally both in nuclear physics and particle physics and clearly seen in exactly solvable models [93] and in full modern continuum shell model applications [94, 95].

The routine identification of the resonance width with the product of a spectroscopic factor (occupancy of a single-particle orbital) and penetrability is invalid being approximately applicable only in the regime of separated resonances. In a more general situation of strong coupling, the width results from repeated excursions to the continuum and back. This should be especially important for many-particle processes as fission and fusion of nuclei [28. Partial widths for specific decay channels also can be calculated, and in the regime of overlapping resonances, the total width $\Gamma_{r}$ is smaller than the sum of partial widths 88 , while the equality holds for well isolated resonances. Another important conclusion is that in general it is not correct to arbitrarily parameterize the set of resonances as poles of the $S$-matrix. This can violate subtle unitarity requirements which lead to correlations of positions and residues at the poles, even for relatively well separated resonances [96.

In strongly bound nuclei, the spectroscopy of low-lying states can be decently described without taking into account the continuum effects, although the widths of resonances and the connection to the scattering problem are lost. In nuclei far from stability the most interesting physics is just on the borderline of the continuum and above. Here, the elements missing in the theoretical foundation of the shell model are to be added phenomenologically, using general physical arguments and available data. The situation is facilitated at low energies and in light nuclei, where only few channels are open. Then a practically reasonable approach is to add a phenomenological potential description to the usual shell model inventory. In some versions of the shell model this is anyway required if the starting point was a harmonic oscillator field and proper radial characteristics are not defined by the model.

\subsubsection{Example: Oxygen isotopes}

A good example can be the shell-model description of the oxygen isotopes 29 , 30, 31. Of course there are promising developments of technically different approaches to similar problems of shell model accounting for the continuum effects [28, 97, 98. Empirically, the last particle-stable oxygen isotope is ${ }^{24} \mathrm{O}$. In the trivial shell model, this would be a core ${ }^{16} \mathrm{O}$ plus eight neutrons completely filling in the levels $0 d_{5 / 2}$ and $1 s_{1 / 2}$. The spin-orbit partner $0 d_{3 / 2}$ is already in the continuum (the neutron width of the $3 / 2^{+}$state in ${ }^{17} \mathrm{O}$ is $96 \mathrm{keV}$ ), while the intermediate level $1 s_{1 / 2}$ is weakly bound, if the level scheme is built on the base of ${ }^{16} \mathrm{O}$. At low energy, and due to the extra stability of the doubly magic 
${ }^{16} \mathrm{O}$, it is reasonable to limit our $\mathcal{Q}$-space by this $s d$-shell model. The relevant decay channels at low energy are single-neutron and two-neutron decays moving the system down along the same oxygen isotope chain. As we mentioned, the knowledge of the decay thresholds is rather critical. To know them we have to study the spectroscopy of the daughter nucleus, and hence to look at the whole chain in its entirety, all neutron-rich oxygen isotopes from the root ${ }^{16} \mathrm{O}$. The two-neutron channels can be restricted by the emission of the correlated pair in the ${ }^{1} S_{0}$ state. It is important to have in mind that for stable states, when the decay widths vanish, this approach automatically reduces to the routine shell model without continuum, maybe with renormalized parameters due to the shift operator $\Delta(E)$.

In practice we need to add to the standard $s d$-model formulation, see for example [99, 100] new parameters describing the two neutron widths under an assumption that only the lowest states of allowed seniority (equal to zero or one) are populated in the daughter nucleus. For excitation energy above $\sim 4$ $\mathrm{MeV}$, one needs to include the final states with higher seniority. The widths for the emission of a $d$-neutron are supposed to grow from the corresponding threshold being proportional to the energy excess to the power $5 / 2$ characteristic for $\ell=2$. The coefficients are found from the neutron scattering problem off the ${ }^{16} \mathrm{O}$ nucleus. Even in the over-simplified framework where the residual interactions were limited to their main components, pairing and monopole terms [29], the results were quite satisfactory indicating the rich perspectives of the whole approach. We have to mention that in principle the effective shell-model interaction could be also complex in the presence of open decay channels; this problem was not solved yet. In calculations of reaction cross sections, the potential phases, especially Coulomb for charged projectiles, can be included along with the dynamics based on the coupling Hamiltonian $H_{\mathcal{P} \mathcal{Q}}+H_{\mathcal{Q P}}$.

The rediagonalization of the effective Hamiltonian for daughter nuclei accounts for the structural rearrangement of the remaining nucleus after neutron decay. Fig. 4 [30] shows the results of the continuum shell model for the chain of oxygen isotopes on the base of the shell model interaction [100. There is a good qualitative and often quantitative agreement with available experimental data. Among interesting predictions, one can mention the ground state width of only $112 \mathrm{keV}$ for the first particle-unbound even oxygen isotope, ${ }^{26} \mathrm{O}$, where the $Q$-value for the two-neutron decay to the last bound isotope ${ }^{24} \mathrm{O}$ is predicted to be only $21 \mathrm{keV}$. In this case only sequential two-neutron decays were considered; this should be extended for correlated pair decays.

\subsubsection{Some technicalities}

A favorable technical feature of working with the effective Hamiltonian is the absence of the need to use explicitly the complex scaling and related tools. One can still keep a diverse experience of the routine shell model and introduce new computational methods for the non-Hermitian Hamiltonian problem 94.

One of such technical improvements is the propagator calculation using the time representation of the propagator 33 for $\mathcal{E} \rightarrow E^{(+)}$as a Fourier image for 
positive time of the Hermitian evolution operator that, in turn, is expressed as an expansion over Chebyshev polynomials [101, $T_{n}(\cos x)=\cos (n x)$,

$$
e^{-i H t}=\sum_{n=0}^{\infty}(-i)^{n}\left(2-\delta_{n 0}\right) J_{n}(t) T_{n}(H) .
$$

The main advantage of the representation is in the long-time dynamics where the Bessel functions $J_{n}(t)$ lead to the convergence important for the energy resolution in the following calculation of the cross sections, strength functions and level densities. Such an approach avoids direct diagonalization of large complex matrices.

With the constructed Green function (33) at real energy and for energyindependent shell-model part $H_{\mathcal{Q Q}}$ of the Hamiltonian, one can solve for the full propagator (32) using the Dyson equation or its equivalent (34) (the socalled Woodbury equation) where the coupling with and through the continuum includes only the matrix in the channel space, usually of a much smaller dimension compared to the intrinsic shell-model space. The full Green function $\mathcal{G}$ allows one to study the damping of quasistationary intrinsic states with non-exponential decay of broad resonances. This approach, supplemented with addition of the collectivizing Hermitian interaction that is absent in the standard $s d$-shell model, also gives a possibility to study giant resonances in loosely bound nuclei, a subject of great interest and contradictory predictions. In this approach the principal value term of self-energy $(29)$ can be included without difficulty and, in practical applications 94 , it improves the description of resonances seen $[102$ in neutron scattering from exotic oxygen isotopes. 


\section{Doorway states}

\subsection{Dynamics based on doorway states}

In a complicated system, it is often the case that only a subset of intrinsic states $|q\rangle$ of space $\{\mathcal{Q}\}$ connects directly to the $\{\mathcal{P}\}$ space of channels. The rest of states in $\{\mathcal{Q}\}$ will connect to $\{\mathcal{P}\}$ only when they acquire admixtures of these selected states of the first type. The special states directly coupled to continuum are the doorways, $|d\rangle$, see for example [103. They form the doorway subspace $\{\mathcal{D}\}$ within $\{\mathcal{Q}\}$, and the corresponding projection operator will be denoted here as $\mathcal{D}$. The remaining states in $\{\mathcal{Q}\}$ will be denoted as $|\widetilde{q}\rangle$ and their subspace as $\{\widetilde{\mathcal{Q}}\}$.

The full Hamiltonian in Eq. 19. can be decomposed now as

$$
\begin{aligned}
H= & \left(H_{\widetilde{\mathcal{Q}} \widetilde{\mathcal{Q}}}+H_{\mathcal{D D}}+H_{\widetilde{\mathcal{Q}}}+H_{\mathcal{D} \widetilde{\mathcal{Q}}}\right) \\
& +\left(H_{\mathcal{P P}}+H_{\mathcal{D P}}+H_{\mathcal{P D}}\right) .
\end{aligned}
$$

Note that the terms $H_{\mathcal{P} \widetilde{\mathcal{Q}}}$ and $H_{\widetilde{\mathcal{Q}} \mathcal{P}}$ are missing because in accordance with the doorway hypothesis they are negligible. Also note that the diagonalization of the Hamiltonian part in the upper line of eq. (68) would provide the states $|q\rangle$ with the components of $|d\rangle$ mixed with the $|\widetilde{q}\rangle$ states. The two last items in the above equation couple the doorway states, and therefore also the $|q\rangle$ states to the open channels.

Doorway states play an important role emphasizing collective effects in description of scattering and reactions with many-body systems. They are often serving as the connection between the continuum and the dense spectrum of quasi-bound states. As we will now see, the concept of the doorway is, in certain situations, closely connected to the notion of super-radiance [41].

\subsubsection{The case of a single doorway}

Let us consider the case when there is only one important doorway state $|d\rangle$. The matrix elements of the effective operator $W$, eq. (30), in the intrinsic space are now given by

$$
\left\langle q|W| q^{\prime}\right\rangle=2 \pi \sum_{c(\text { open })}\left\langle q\left|H_{\mathcal{D P}}\right| c\right\rangle\left\langle c\left|H_{\mathcal{P} \mathcal{D}}\right| q^{\prime}\right\rangle
$$

with the doorway assumption:

$$
\left\langle q\left|H_{\mathcal{D P}}\right| c\right\rangle=\langle q \mid d\rangle\left\langle d\left|H_{\mathcal{D} \mathcal{P}}\right| c\right\rangle,
$$

where $\langle q \mid d\rangle$ is the amplitude of the admixture of the doorway into the $|q\rangle$ state. Eq. 70 now becomes:

$$
\left\langle q|W| q^{\prime}\right\rangle=2 \pi\langle q \mid d\rangle\left\langle d \mid q^{\prime}\right\rangle \sum_{c(\text { open })}\left|\left\langle d\left|H_{\mathcal{D P}}\right| c\right\rangle\right|^{2},
$$


where again we have separable matrix elements of the matrix $W$, this time irrespective of the number, $M$, of open channels. The doorway serves as a filter for the coupling of the $\{\mathcal{Q}\}$-space to the space of open channels $\{\mathcal{P}\}$. As a result, we find one broad state with a width

$$
\Gamma_{d}^{\uparrow}=2 \pi \sum_{q}|\langle q \mid d\rangle|^{2} \sum_{c}\left|\left\langle d\left|H_{\mathcal{D P}}\right| c\right\rangle\right|^{2}=2 \pi \sum_{c}\left|\left\langle d\left|H_{\mathcal{D P}}\right| c\right\rangle\right|^{2} .
$$

This width is nothing but the decay width of the doorway into all available channels.

\subsubsection{When is this approach valid?}

As discussed above, the criterion of validity of the super-radiant mechanism is that the average spacing between the levels in $\{\mathcal{Q}\}$-space is smaller than the decay width of a typical state "before" the super-radiant mechanism takes effect. This can be expressed, in the case of doorways, in the following way 41.

Consider the spreading width, $\Gamma_{d}^{\downarrow}$, of the doorway state representing the fragmentation of $|d\rangle$ into compound states $|\widetilde{q}\rangle$. If $N_{q}$ is the number of compound states in the interval covered by the spreading width, their average energy spacing is

$$
D_{q} \approx \frac{\Gamma_{d}^{\downarrow}}{N_{q}}
$$

Before the super-radiant mechanism is turned on, the average decay width of a typical $|q\rangle$ state is

$$
\Gamma_{q}^{\uparrow}=2 \pi \sum_{c}\left|\left\langle q\left|H_{\mathcal{Q P}}\right| c\right\rangle\right|^{2}
$$

which can be estimated by

$$
\Gamma_{q}^{\uparrow} \approx \frac{\Gamma_{d}^{\uparrow}}{N_{q}}
$$

These estimates result in

$$
\frac{\Gamma_{q}^{\uparrow}}{D_{q}} \approx \frac{\Gamma_{d}^{\uparrow}}{\Gamma_{d}^{\downarrow}}
$$

We conclude that the requirement for the super-radiant doorway mechanism to be valid can be formulated as

$$
\frac{\Gamma_{d}^{\uparrow}}{\Gamma_{d}^{\downarrow}}>1
$$

the decay width of the doorway should be comparable or larger than its spreading width in order for the super-radiant mechanism to be valid. This condition is not always obeyed, but in some cases, as we will see, it is satisfied. 


\subsubsection{Isobaric analog resonances}

An outstanding example, where the condition (77) is fulfilled and the superradiant mechanism may take place, is given by the isobaric analog state (IAS) $|I A S\rangle$ [104] that is obtained from the parent state $|\pi\rangle$ with certain isospin $T$ by acting with the isospin lowering operator $T_{-}$that changes a neutron into a proton:

$$
|I A S\rangle=\text { const } \cdot T_{-}|\pi\rangle .
$$

In medium and heavy nuclei, the IAS are surrounded by many compound states $|q\rangle$ of lower isospin $T_{<}=T-1$. The Coulomb interaction violates the isospin symmetry fragmenting the strength of the IAS over many states $|q\rangle$ and giving rise to the spreading width $\Gamma_{I A S}^{\downarrow}$ of the IAS. If located above thresholds, the IAS can also decay into several continuum channels with the decay width $\Gamma_{I A S}^{\uparrow}$. In such cases the condition (77) is usually satisfied. For example, in ${ }^{208} \mathrm{~Pb}$ the spreading width of the IAS is about $80 \mathrm{keV}$ while the escape width is about $160 \mathrm{keV}$ [104], thus the ratio $\Gamma_{I A S}^{\uparrow} / \Gamma_{I A S}^{\downarrow} \approx 2$. This situation is typical for other heavy and medium mass nuclei. In the $Z \sim 40$ region the above ratio is in some cases even larger than 2 [104]. The super-radiant mechanism is therefore relevant to this case providing a straightforward explanation why the IAS appears as a single resonance with the decay width of the doorway $|I A S\rangle$,

$$
\Gamma_{I A S}^{\uparrow}=2 \pi\left|\left\langle I A S\left|H_{\mathcal{Q P}}\right| \mathcal{P}\right\rangle\right|^{2}
$$

\subsection{Giant resonances}

Giant resonances in nuclei (or atomic clusters) present another example of similar physics [105, 106]. Usually, the giant resonances are discussed in terms of particle-hole $(p-h)$ configurations with identical spin-parity quantum numbers. The coherent residual interactions form a correlated state that carries much of the transition strength of a corresponding multipole operator. The giant resonances are mostly located in the particle continuum decaying via particle emission to the ground and excited states in the daughter nucleus.

Since the $p-h$ giant resonance $|G\rangle$ is surrounded usually by a dense spectrum of $2 p-2 h$ and more complex configurations, the residual strong interaction will mix the state $|G\rangle$ with this background. Each of the resulting states denoted as $|b\rangle$ will contain the admixture $\langle G \mid b\rangle$ of the giant resonance. When above the threshold, the mixed states $|b\rangle$ couple to the continuum. If we assume that the dominant coupling is via the admixture of the giant state, then $|G\rangle$ serves as a doorway, and the matrix $W$ is separable, given by

$$
\left\langle b|W| b^{\prime}\right\rangle=\langle b \mid G\rangle\left\langle G \mid b^{\prime}\right\rangle \sum_{c}\langle G|A| c\rangle\langle c|A| G\rangle,
$$

where $\langle G|A| c\rangle$ are the decay amplitudes for the giant resonance state. The matrix $\left\langle b|W| b^{\prime}\right\rangle$ is again of rank one and the non-zero eigenvalue is equal to

$$
\Gamma_{G}^{\uparrow}=2 \pi \sum_{c}|\langle G|A| c\rangle|^{2}
$$


This is valid under the assumption that the energy spread of the background states $|b\rangle$ is small compared to their decay width,

$$
\Gamma_{b}^{\uparrow}=2 \pi \sum_{c}|\langle b|A| c\rangle|^{2} .
$$

As before, this condition can be expressed as

$$
\frac{\Gamma_{G}^{\uparrow}}{\Gamma_{G}^{\downarrow}}>1
$$

the spreading width of the giant resonance is smaller than its total decay width.

When the condition (83) is not satisfied, so that the energy intervals between the background states are larger than their decay widths $\Gamma_{b}^{\uparrow}$, the situation of a single decay peak for the giant resonance might not hold. Still one could expect some bunching of background states into groups with spacing within the group smaller than the decay widths. Each such a group then can be treated separately using the super-radiant mechanism and appear as a single peak in the decay (or excitation) curve. One will then observe intermediate structure resonances in the giant-resonance energy domain.

One should remark that for many giant resonances, such as the giant dipole resonance (GDR) or giant isoscalar quadrupole resonance, the above condition (83) is not fulfilled [105, 106, and the spreading width of the resonance exceeds the decay width. However, in continuum RPA calculations it was found that high lying giant resonances have substantial decay widths [105], larger than their spreading widths. For example, the isovector monopole resonances calculated in heavy nuclei had decay widths of the order of 8-10 MeV, larger than the spreading widths which are of the order of a few $\mathrm{MeV}$. In these cases the condition 82 for super-radiance is satisfied.

The GDR in nuclei not far from the valley of stability is shifted up in energy compared to the unperturbed excitation energy $\sim 1 \hbar \omega$ of $p-h$ states. The GDR strength then saturates a significant part of the classical dipole sum rule although some strength still remains around the unperturbed energy. The situation changes for nuclei with a large neutron excess farther away from stability. The fraction of dipole strength in $p-h$ states that are not shifted increases due to the possibility of cooperative oscillation of the neutron excess against the core. If in an unstable nucleus some of these states are already in the continuum, they interact not only by the residual dipole-dipole interaction but through common decay channels. Then the situation reminds what we have discussed for the main branch of the GDR. As a result, the so-called pygmy-branch of the giant resonance [107, 108] is formed being currently a subject of serious experimental interest. This soft collective dipole mode serves again as a doorway state. Such a mechanism of formation of the pygmy-resonance was predicted in [109] and considered more in detail in [110].

In the limit of small energy spread of the $p-h$ states, the interplay and redistribution of dipole strength and decay width to a channel $c$ between the 
main GDR and the pygmy-resonance is determined by the "angle" between the multidimensional vectors $\mathbf{A}=\left\{A_{q}^{c}\right\}$ of decay amplitudes and $\mathbf{d}=\left\{d_{q 0}\right\}$ of dipole matrix elements connecting $p-h$ excitations and the ground state. If these vectors are "parallel", the soft mode loses its collective character, and the main GR peak concentrates both the strength and the continuum width. A situation of this type takes place in the case of the collective $\Delta$-isobar-nucleon-hole states, discussed in the Section 5.2, when the pion matrix elements determine both the strength and the width [38, 111] of the doorway state. In the extreme "orthogonal" case the main peak would concentrate the multipole strength but the whole width would go to the soft mode so that the main peak would become a "zombie" state embedded in the continuum. The reality corresponds to an intermediate situation with the weight of the pygmy-branch rising closer to the drip line. In such cases it makes sense to speak about exit doorways [112, 113].

\subsection{Several channels and intermediate structure}

Let us turn to the situation when the $|q\rangle$ states are coupled to a number of open channels in the $\mathcal{P}$-space, a typical situation in nuclear physics. In addition to the elastic channel, the compound states in many cases can decay to excited states in the daughter nuclei. In this situation we have a number of open channels $M$ that is still much smaller than the number of $|q\rangle$ states. In many cases it is not the same doorway that connects to all the open channels. In fact often the doorways are specified, and their number is equal to the number of open channels 41 .

We should point out the difference between the situation mentioned here and the case of a single doorway for all the channels discussed above. In the latter case the matrix $W$ was factorized, eq. 700 , as $W_{q q^{\prime}}=a_{q} a_{q^{\prime}}^{*}$ but the present case is more complicated because each channel has its own doorway, so that the matrix $W$ is of the form

$$
W_{q q^{\prime}}=\sum_{d=1}^{M} a_{q}^{d} a_{q^{\prime}}^{d *}
$$

When the number $|q\rangle$ states is much larger than the number of channels $M$, correlations are present in the matrix $W_{q q^{\prime}}$. In this case the cross section will exhibit several resonances, with intermediate widths $\Gamma_{\text {int }}$ such that $\Gamma_{q} \ll \Gamma_{\text {int }}<\Gamma_{\text {s.p. }}$, where $\Gamma_{\text {s.p. }}$ is the decay width of a single-particle resonance. Such intermediate resonances were observed in nuclear reactions long ago [40, 41] and the cross sections were said to exhibit intermediate structure.

In some situations, several doorway states $|d\rangle$ appear separated by energy intervals larger than their total widths. The intrinsic states $|q\rangle$ couple to these doorways locally giving rise to spreading of doorways. According to the doorway hypothesis, the $|q\rangle$ states couple to the continuum only via the admixture(s) of the local doorways. Then the matrix $W$ separates into several disconnected quadratic blocks of dimensions $n_{d} \times n_{d}$, where $n_{d}$ is the number of states $|q\rangle$ contained within the spreading width of the doorway $|d\rangle$. For each such a sub-matrix, eq. (71) is valid, and the problem is reduced to that of the single 
doorway. The resulting picture is a series of resonances separated in energy with decay widths equal to the decay widths of the individual doorways.

\subsection{Highly excited doorway state}

As excitation energy grows, many open decay channels are getting accessible. This region has its own specific features. A doorway state is singled out by a specific "signal", either a quantum number different from that of the majority of background states, or by a character of intrinsic motion and, correspondingly, a type of external excitation.

A nuclear example of the first kind is given by the IAS that is a state of isospin $T_{>}=T$ that is embedded into a sea of states of isospin $T_{<}=T-1$. The Coulomb interaction and a small component of nuclear forces violating charge independence mix the IAS to the background. This spreading width of the IAS increases with excitation energy and then saturates [114. If at this excitation energy the lifetime of the $T_{>}$state with respect to the decay outside is shorter than the lifetime of the background $T_{<}$states, the IAS preserves its individuality [15, 116. One could expect that the purity of the IAS will rapidly be washed out at high density of the admixed states. As it turns out, just opposite is correct: after increased mixing, at some energy the purity of the IAS is restored as confirmed experimentally [117. In fact, this phenomenon was predicted long ago by Morinaga [118 and Wilkinson [119. A qualitative explanation follows from plausible kinetic arguments [120]. The fully quantum-mechanical doorway theory [121] naturally justifies this conclusion.

Let the doorway state $|d\rangle$ be immersed into a rich set of $N \gg 1$ background states $|\nu\rangle$. The state $|d\rangle$ with complex energy $\mathcal{E}_{0}=\epsilon_{0}-(i / 2) \gamma_{0}$ can decay into continuum (the corresponding width is $\gamma_{0}$ ) and to be mixed with background states $|\nu\rangle$ by the coupling matrix elements $V_{\nu}$ which can be considered as real. In turn, the background states, after diagonalizing their intrinsic coupling, can at this energy decay into continuum through many, $M \gg 1$, complicated and essentially uncorrelated evaporation channels; their complex energies are $\epsilon_{\nu}$. Because of the complexity of those states and presence of many open channels, the coherent super-radiant collectivization of the background states is suppressed. The coupling of the doorway state with the background defines new quasistationary states $|j\rangle$, and, similarly to the usual theory of bright stationary states, the complex energies $\mathcal{E}_{j}$ are the roots of

$$
\mathcal{E}_{j}-\mathcal{E}_{0}-\sum_{\nu} \frac{V_{\nu}^{2}}{\mathcal{E}_{j}-\epsilon_{\nu}}=0 .
$$

Each root $j$ with energy $\mathcal{E}_{j}=E_{j}-(i / 2) \Gamma_{j}$ carries a fraction $f_{j}$ of the doorway strength, $\sum_{j} f_{j}=1$. Substituting the evaporation widths by their typical value $\gamma_{\mathrm{ev}}$ and calculating the imaginary part of eq. 85, we find [121]

$$
f_{j}=\frac{\Gamma_{j}-\gamma_{\mathrm{ev}}}{\gamma_{0}-\gamma_{\mathrm{ev}}}
$$


This result has a clear probabilistic, or kinetic, meaning indicating two routes of each fine structure resonance to the irreversible decay,

$$
\Gamma_{j}=\gamma_{0} f_{j}+\gamma_{\mathrm{ev}}\left(1-f_{j}\right) .
$$

The further analysis shows the resulting picture and its evolution with excitation energy from the viewpoint of reaction cross sections and time delays. The characteristic parameter here is the ratio $\gamma_{\mathrm{ev}} / D \sim N \gamma_{\mathrm{ev}} / \Gamma^{\downarrow}$ of the evaporation width to the spacing of compound states; the spreading width of the doorway state is $\Gamma^{\downarrow} \sim N D$. Instead of super-radiance in this situation of many uncorrelated open channels, when the background states overlap, the fluctuations in the excitation of the doorway state are washed away, see Fig. 5, and the depletion of the admixed background states of opposite isospin occurs faster than their population through the doorway mechanism. This justifies the predictions of isospin purification [118, 119] observed in [117]. Such effects can be also interpreted as related to the quantum Zeno effect [122. In kinetic language of Ref. [120], this stage corresponds to temperature of the compound nucleus exceeding a critical value when the total evaporation width of the admixed states becomes of the order of the spreading width $\Gamma^{\downarrow}$ of the IAS. In a similar formalism, analogous hierarchical effects were considered [123, 124] for chaotic quantum dots or atoms coupled to the electromagnetic modes of a cavity immersed in a bigger box. At random coupling, one can see mesoscopic fluctuations and oscillations of the survival probability.

Another related effect is the so-called disappearance of collective strength of giant resonances excited in a high-energy heavy ion collision [125]. The analogs of giant resonances are expected to exist being built on all other nuclear states (Axel-Brink hypothesis). However, beyond a critical excitation level, excited in heavy ion collisions fine structure states, according to the mechanism discussed above, decay in the continuum through incoherent evaporation channels before they are capable to transfer excitation to a collective degree of freedom: in a sense, the original excitation has no time to open the door through the doorway.

\subsection{Additional comments}

We have considered few examples justifying a generic qualitative picture of formation of doorway states and intermediate structures in various nuclear processes. It would be interesting to extend this approach to other mesoscopic systems (the application of the doorway concept to giant resonances in atomic clusters was made in 126). The notion of the doorway state has been applied to atoms and molecules [127, to quantum dots [128, and fullerenes [129, 130] and recently even to a classical phenomenon of earthquakes [131. In the physics of cold atoms [132, the coupling of the internal states to the continuum via the Feshbach resonance influences profoundly the system affecting not only the imaginary part of the effective Hamiltonian but also the real part. This enables one to manipulate the properties of the system in a trap by changing the sign and/or the strength of the two-body interaction. The Feshbach resonance in this case serves as a doorway. 
The traditional theory of intermediate structure in nuclear reactions [133, 134 considers usually statistical sequences in time of multi-step particle interactions with possible pre-compound (or pre-equilibrium) decay to the continuum. The present formulation emphasizes complementary aspects of underlying physics, namely those that follow from the strict quantum-mechanical description of complicated many-body dynamics generated by the mean field and residual interactions. The approach based on the effective non-Hermitian Hamiltonian rigorously predicts the existence of different scales in the energy dependence of observed cross sections. These scales are defined by the intrinsic dynamics that combines collective (coherent) and chaotic (incoherent) features. The new collectivity responsible for the emergence of the doorways and related intermediate structure appears, under certain physical conditions, due to the factorized nature of corresponding terms in the effective Hamiltonian. This separability is deeply related to the properties of unitarity of the scattering matrix. In this way we obtain a supplementary description of many-body dynamics. 


\section{Other applications}

\subsection{Atomic and molecular physics}

Many-body physics of complex atoms has many features in common with nuclear many-body physics - shell structure, residual interactions, growth of complexity of excited states with excitation energy, very complicated - "compound" - wave functions of mixed states with several excited electrons, presence of the continuum etc. This structure and high level density are revealed, in particular, in the photoabsorption spectrum, see for example, [135, 136]. Typical autoionizing states appear already in the set of two-electron excitations.

The whole super-radiant formalism was translated to the language of atomic physics in Ref. 35. The physical difference from analogous nuclear phenomena is mainly due to the long-range character of the electrostatic interactions. In nuclear neutron resonances typical spacings between the resonances are greater than their widths, and the super-radiant collectivization requires higher energy above the neutron separation threshold, where the effect might be overshadowed by the opening of new incoherent channels. In atoms the influence of threshold factors, $\sim k R$, is suppressed by the Coulomb effects, which facilitates the appearance of collectivization through continuum. According to [35], the photoabsorption experiments will be mostly sensitive to narrow trapped states; in atoms the intrinsic collectivity similar to that generating giant resonances in nuclei is substantially weaker. But in electron scattering from ions one should observe broad doorway states and, with sufficient resolution, narrow trapped states as well. This difference is clearly seen in numerical calculations 35.

Experimental data mainly received with multiphoton laser excitation of atomic beams confirm the trend to the formation of trapped states with the autoionization rate suppressed compared to the predictions for a simple atomic configuration [137, 138]. Ref. [139] provides a detailed review of this research area.

The practical calculations of atomic spectra in principle can be done with the aid of the same shell-model machinery (currently called by the term "configuration interaction" coined in quantum chemistry) as in nuclei, especially because the interaction is essentially known. A new element comes from the presence in the same energy region, along with complicated compound states, also the Rydberg states of simple nature. The Rydberg spectrum is condensed near every ionization threshold. However, the large difference in structure makes the mixing between compound autoionizing states and Rydberg autoionizing states weak being in addition strongly suppressed by their very different radial behavior. The real spectrum consists therefore of two weakly mixing subsets.

Interesting physics appears in the case of a simple (for example, hydrogen) atom in a strong uniform magnetic field [140. The bound states in the continuum can be found according to the results of our two-level problem, Sec. 4.1. In the field around the critical value, $B_{\mathrm{cr}}=m^{2} e^{3} c^{3} \hbar^{3} \sim 10^{5} \mathrm{~T}$, the cyclotron energy $\hbar \omega_{\mathrm{c}}$, and therefore, the splitting of Landau levels, are of the same order as atomic Rydberg energy $m e^{4} / \hbar^{2}$. The Rydberg series overlap, 
and the sequences of Landau levels in the continuum interfere. This creates the situation of super-radiation and trapping, and, for some values of parameters (magnetic field), bound states in the continuum. It was stressed [141] that this phenomenon, earlier found in specifically adjusted potentials, is more general leading to segregation of fast decaying and trapped states.

In atomic physics one can directly calculate the necessary matrix elements between the internal wave functions and continuum decay channels. An approximate statistical treatment [35] of the states $J^{\pi}=4^{-}$in the cerium atom showed that these matrix elements have the Gaussian-type distribution that leads to the Porter-Thomas distribution (PTD) of the resonance widths. Later, Section 7, we discuss the recent experimental indications and corresponding theoretical arguments for nuclear neutron resonances which show generic deviations from the PTD. In atoms the presence of two components of the spectrum may distort the picture.

Radiationless and multiphoton transitions in atoms and molecules present an appropriate applications of super-radiant physics. The effect of super-radiance and trapping in molecular transitions in the approximation of degenerate intrinsic levels coupled to the same decay channel was considered in [36] and possible generalization to non-degenerate levels was mentioned (called in this paper "quasitrapping"). The actual presence of fast and slow dynamics in the multiphoton excitation was noticed long ago [142].

\subsection{Intermediate and high energy physics}

As discussed in previous sections the super-radiant mechanism was applied to a number of different systems involving many-body physics. The phenomena discussed in the field of nuclear physics involved low energies. The universality of the super-radiant mechanism leads us to consider also the field of intermediate energy phenomena. The first discussion concerning this can be found in Ref. [37. In this work the super-radiant mechanism was applied to a number of situations involving intermediate energy hadronic systems. The main objective of these studies was to point out that narrow resonances can be formed due to the super-radiant mechanism. Here we bring some examples.

\subsubsection{The $\bar{N} N$ states}

In current relativistic theories 143 of nuclear structure the Dirac vacuum of occupied negative energy states plays an important role in describing some of the nuclear properties. The influence of these negative energy states, $\bar{N}$, is often implicit. It was suggested [144] that such theories could be tested by an observation of particle-hole states, in which a nucleon is raised from the negative energy Dirac sea and placed in an unoccupied state with positive energy. That we call an $\bar{N} N$ excitation. In most relativistic models the nucleon occupying a negative energy state experiences a strong potential of the order of several hundred, up to 700-800, MeV. Thus the excitation energy of the $\bar{N} N$ state is

much lower than the mass of two nucleons, $E_{\bar{N} N}<2 M_{N}$. The observation of 
such sub-threshold nucleon-antinucleon excitations would provide a direct test of the relativistic theories of nuclei. A question should be raised about the feasibility to observe such excitations because of the expected large annihilation width. The $\bar{N} N$ pair is annihilated into mesons, in particular into several pions. One can expect that the $\bar{N} N$ states will couple strongly to the annihilation channel and decay very quickly. The large widths of these states will hamper their observation.

It is worth noting however that conditions for the formation of a superradiant state exist in this case 144. The spectrum of negative energy states is within a given shell. (The spin-orbit splitting is nearly zero for the negative energy states). The unoccupied positive energy states form also a dense spectrum, and therefore one expects a strong degeneracy among the unperturbed $\bar{N} N$ configurations. The energy splitting between various $\bar{N} N$ states is typically $5-10 \mathrm{MeV}$, thus smaller than the individual decay widths into the common annihilation channel. We are therefore in the regime of overlapping resonances.

Using a relativistic random phase approximation (RPA), a calculation of energies and widths of $\bar{N} N$ states was performed in the framework of the $\sigma-\omega$ model [145] for ${ }^{16} \mathrm{O}$. The width was obtained from an imaginary term in the meson propagators that mimicked the coupling to the annihilation channel. The result of the diagonalization of the complex RPA matrices is quite amazing. All the states that resulted from the diagonalization had width of the order of 1-2 $\mathrm{MeV}$, while one state had a width of about $160 \mathrm{MeV}$. This is the super-radiant state. What happens here is that each $\bar{N} N$ state decaying to the common channel produces width of the order of $15 \mathrm{MeV}$. As a result of the diagonalization of the matrix, the wide super-radiant state emerges while the rest of states become very narrow and thus live a long time. One could anticipate the following situation. In a simple reaction, as for example nucleon pick-up, the super-radiant resonance, because of its large width will show up as a flat bump contributing to the background. On top of this background, narrow peaks will appear corresponding to the $\bar{N} N$ states that were "stripped" of their width by the super-radiant resonance.

\subsubsection{The $\Delta$-Nucleus system}

Several years ago an experiment on inelastic electron scattering was performed with the reaction ${ }^{12} \mathrm{C}\left(e, e^{\prime} p \pi^{-}\right)^{11} \mathrm{C}$. Evidence was found for narrow (several $\mathrm{MeV}$ wide) resonances in the $\Delta_{33}+{ }^{11} \mathrm{C}$ system in addition to a broad peak of approximately $100 \mathrm{MeV}$ width [146].

The free pion-nucleon resonance $\Delta_{33}$ with spin $3 / 2$ and isospin $3 / 2$ has a total width of $\Gamma_{\Delta}=120 \mathrm{MeV}$, and the centroid of the four charge components is at $1235 \mathrm{MeV}$; this is $300 \mathrm{MeV}$ above the nucleon mass. The excitation of nuclei with various medium energy probes (electrons, protons, etc.) reveals a wide peak around the energy of $300 \mathrm{MeV}$. This peak is described in the framework of $\Delta$ particle-nucleon hole $\left(\Delta N^{-1}\right)$ configurations [111. The $\Delta$-component is treated as a particle bound in a potential that has similar characteristics to the usual nucleon potential. The nuclear $\Delta$ resonance is determined by the 
coupling of the $\Delta N^{-1}$ configurations to the decay channel that involves pion emission. The $\Delta$-nucleus system has properties that make the application of the super-radiant mechanism plausible. The $\Delta N^{-1}$ configurations are strongly coupled to a single channel $|c\rangle=\left|A-1 ;(N+\pi)_{\Delta}\right\rangle$, corresponding to a (nucleon plus pion)-system with quantum numbers of the $\Delta$ resonance and the residual $(A-1)$ nucleus. The partial decay width of each $\Delta N^{-1}$ state is a fraction of the total width of the free $\Delta_{33}$, of the order of a few tens of MeV. On the other hand, the energy spacing between these states is of the order of several $\mathrm{MeV}$. We are dealing here with the case of overlapping resonances.

In Ref. 38, a simple shell-model description was used for the ${ }^{12} \mathrm{C}$ target nucleus. The nucleons occupy the lowest shell-model orbits $0 s_{1 / 2}$ and $0 p_{3 / 2}$ orbits. The $\Delta$ particle is assumed to move in a potential similar to the mean nuclear field. Therefore the energy spacings between the nuclear orbits and between the $\Delta$ orbits are close. One can then form $\Delta N^{-1}$ states for various total spins $J$. For example, there are four $\Delta N^{-1}$ states with $J=1^{+}$. Applying the theory of super-radiance we should find three very narrow states (with widths of several $\mathrm{MeV}$ ) and one very broad resonance with a width around $100 \mathrm{MeV}$. The other states with spins $J=2^{+}$and $3^{+}$will add some narrow peaks. This picture resembles very much the observed cross-section [146]. One should bear in mind that other mechanisms might contribute to the observed widths of these quasistationary states. For example, the nucleon-hole states acquire spreading widths due to the residual nucleon-nucleon interaction. It is important to confirm the experimental results of Ref. 146 and try to study the possible narrow structure using alternative reactions that excite the $\Delta$-nucleus resonances.

\subsubsection{High energy phenomena}

In many applications of the super-radiant mechanism, including the last two cases, the emphasis was placed not only on the wide super-radiant state, but also on the fact that the mechanism of the theory creates narrow resonances superimposed on a background formed by the broad super-radiant state, leading in this way to the separation of fast (direct) processes from the long-lived structures. The consequences of this were examined and it was suggested that a mechanism of this type can explain the existence of narrow auto-ionizing states in atoms and narrow resonances in a number of strongly interacting hadronic systems.

In the years 2003-2004 evidence was brought for the existence of a narrow baryon resonance with strangeness $S=+1$ around energy $E=1540 \mathrm{MeV}$ 147, 148. It was found in various experiments that the width of this resonance (coined as $\Theta^{+}$or $Z^{+}$), was a few $\mathrm{MeV}$ only. The resonance was situated on top of a very broad (over $100 \mathrm{MeV}$ ) background peak. It was difficult to understand the existence of this very narrow resonance structure in the framework of the usual mechanism for decay of baryonic resonances, whether exotic or non-exotic. The narrow width of the $S=+1$ exotic baryon indicates that the decay into the kaon-nucleon continuum is quenched either by selection rules, for example if the $\Theta^{+}$is an isotensor, or by some dynamical features. 
In view of our previous discussions, it is possible that the narrowness of the peak observed is a result of the super-radiant mechanism [39. This case serves as a direct example of the two-level super-radiant formalism discussed in Section 4.1. In the simplest scenario one can consider the kaon-nucleon pentaquark system ( $s \bar{u}+d d u$ quarks) to be nonrelativistic and to form a quasi-molecule. Molecular-like structures for non-strange pentaquarks were discussed by Iachello [149. The two particles interact via an attractive potential of a typical range of $1 \mathrm{fm}$ producing a $p$-wave resonance at energy $100 \mathrm{MeV}$ above threshold. In Ref. [150] the authors estimate that the width of the resonance in such a potential is greater than $175 \mathrm{MeV}$, the value typical for strong decays of baryons in this mass region. A direct calculation gives the $p$-resonance width $190 \mathrm{MeV}$ for the well of radius $a=1 \mathrm{fm}$ obtained at the relevant energy; this required the depth of the well to be $V=333 \mathrm{MeV}$; the width of $327 \mathrm{MeV}$ is obtained for $a=2$ fm and $V=14 \mathrm{MeV}$. In addition to quasi-molecular states, one should consider many-quark bag dynamics in a system of five quarks formed after the photon absorption by the original proton. Among the intrinsic states there are groups with the same quantum numbers, including "normal" quark states, quark-gluon states, paired states with singlet or triplet diquark(s), states with pions and so on. If the decay width of each individual state is greater than the energy spacing, the unperturbed spectrum will be that of overlapping resonances. Under such conditions, the dynamics will be completely dominated by the coupling to the decay channel, and the anti-Hermitian part of the Hamiltonian will be the crucial factor. As a result, the super-radiant mechanism will give rise to the observed spectrum with a very broad, $\sim$ several hundred $\mathrm{MeV}$, background peak of the super-radiant state and one or several very narrow resonances with width of order of few $\mathrm{MeV}$. For example, for two overlapping resonances with $\Delta E \approx 20$ $\mathrm{MeV}$, and the bare width of $\gamma_{1,2} \approx 200 \mathrm{MeV}$, using the equations of Sec. 4.1, the estimate gives for the narrow resonance $\Gamma_{\text {narr }} \approx 4 \mathrm{MeV}$. A more detailed and quantitative account of this can be found in Ref. [39. In Ref. 151] the authors proposed somewhat different candidates for the two interacting states. In summary, due to the overlap of unstable intrinsic states, the super-radiant mechanism may produce the narrow peak(s) on the broad background in exotic baryon systems with strangeness +1 .

We should remark here that the observation of pentaquarks is presently quite controversial. Some experiments that followed the initial claims of observation have reported the absence of pentaquark signals close to energy of $1540 \mathrm{MeV}$. Also some of the experiments that did report initially evidence for the existence of pentaquark have now retracted these claims [152]. Nevertheless the theoretical discussion above is still valid. The assumption of the existence of two states close in energy around $1540 \mathrm{MeV}$ might not be fulfilled. Generically, however it is possible to obtain a very narrow width for high energy resonances when there are favorable conditions for the super-radiant mechanism.

In the last several years, in addition to the pentaquark, a plethora of papers were published (mainly from the $B$-factories) reporting the observation of new resonances [153, 154, 155] with small decay widths, much less than typical hadrons would have at these energies (some of the reported resonances had 
widths of a few MeV only). Experimental and theoretical studies suggest that among these narrow resonances might be tetraquarks, that is systems with two quarks and two anti-quarks. These new resonances mostly lay around the mass region of $4 \mathrm{GeV}$, and the proposal is that they are composed of a light quarkantiquark pair and a heavy quark-antiquark pair, as for example $c \bar{c} u \bar{u}, b \bar{b} u \bar{u}$, and $c \bar{c} d \bar{d}$. Heavy tetraquarks are expected to decay into heavy states (as for example the $J / \psi$, charmonium) and light $q \bar{q}$ states, for example pions. The decay width of such processes is expected to be large, of the order of hundreds of $\mathrm{MeV}$. However, in view of what we have discussed above, if there are two closely spaced tetraquark states with the same quantum numbers and both are coupled to the same final decay mode, there will be a rearrangement of widths, one state will become very wide while the other will become narrow.

The most studied, so far, resonance among the ones suspected to be tetraquarks, is the $X(3872)\left[153,154,155\right.$, which decays into $(J / \psi) \pi^{+} \pi^{-}$. The decay width is very small, $\Gamma<2.3 \mathrm{MeV}$. An unusually small width is an indication of strong incoherence and cancelation, suggesting that the super-radiant mechanism might be responsible for this. The broad (super-radiant) state has a very large width and is embedded in the background. There are several observations of additional resonances with widths not as small as that of $X(3872)$, but still narrow compared to the typical characteristic decay widths in this energy region.

The Belle collaboration reported recently the observation of the $Z(4430)$ particle [156. This particle is unusual because it has non-zero charge therefore providing a strong hint that it is a tetraquark. The dominant component of its wave function is probably $c \bar{c} u \bar{d}$ that decays into $\psi^{\prime} \pi$. The decay width is of the order of $45 \mathrm{MeV}$. This again is an anomalously small value, especially considering the fact that the state is located several hundred $\mathrm{MeV}$ above threshold. It could possibly be viewed as a realization of the super-radiant mechanism. One should cautiously approach the new discoveries of tetraquark states and seek more experimental and theoretical confirmation before drawing certain conclusions about the mechanisms responsible for narrow resonance widths. The approach outlined here presents one of possible candidates to explain these long-lived resonances. 


\section{Quantum chaos in an open system}

\subsection{Many-body quantum chaos}

Our discussion of doorway dynamics with separation of scales corresponding to external and internal interactions naturally leads to a general problem of quantum chaos. The deterministic chaos in classical mechanics is now a well understood and discussed in textbooks [157] phenomenon based on the extreme sensitivity of phase space trajectories to small variations of initial conditions. Contrary to that, quantum chaos [158 still is a subject of discussions, and some authors deny its existence recognizing only quantum signatures of classical chaos [159]. In spite of this, we can formulate generic manifestations of quantum chaotic dynamics in a stationary quantum system as the presence of local correlations and fluctuations of observables approaching the extreme mathematical limit of canonical Gaussian ensembles of random matrices [160].

One-body quantum chaos emerges in systems of billiard type being caused by the boundary conditions, for example coming from an asymmetric shape that destroys spatial symmetries [161. Such dynamics can be successfully reproduced experimentally with microwave cavities [162, especially in the low-temperature superconducting regime with suppressed thermal dissipation [163. In condensed matter systems, chaotic dynamics can be generated by the presence of disorder [164] or external fields [165] and, therefore, can be experimentally regulated.

Our main interest is in physics of many-body quantum chaos where the chaoticity is originated by interparticle interactions 166. As excitation energy and level density increase and any residual interaction mixing the independent mean-field particle configurations becomes effectively strong, many-body quantum dynamics in self-bound systems approaches chaotic limit. Then the stationary states develop into exceedingly complicated superpositions of original simple configurations. The process of chaotization was tracked down in detail for complex atoms [167, nuclei [168] and other mesoscopic objects.

The ideal characteristics of quantum chaotic dynamics are given by the predictions of the Gaussian orthogonal ensemble, GOE, (or Gaussian unitary, GUE, for the case of violated time-reversal invariance, and Gaussian symplectic, GSE, applicable, in particular, to time-reversal invariant systems with an odd number of fermions and therefore Kramers degeneracy) [169, 160, 170, 171]. The distributions of energy eigenvalues in these ensembles are invariant with respect to corresponding (orthogonal, unitary, or symplectic) transformations of the basis and separated from the distributions of the components of eigenvectors,

$$
P_{\beta}\left(E_{1}, \ldots, E_{N}\right)=C_{\beta}(N) \prod_{m<n}\left|E_{m}-E_{n}\right|^{\beta} \exp \left[-\beta N \sum_{n} E_{n}^{2} / a^{2}\right] .
$$

Here $C_{\beta}(N)$ are normalization constants, $\beta=1,2,4$ refer to GOE, GUE, and GSE, respectively, while $a$ is the only available scale, a measure of the energy spread of the whole spectrum under consideration. Typical features of such distributions are well known: level repulsion at small spacings (linear, quadratic 
and quartic for those ensembles as can be understood from elementary quantummechanical perturbation theory [172]), small probability of large gaps in the spectrum, and the maximum of the probability close to the mean level spacing so that the levels in this limit form an aperiodic crystal with suppressed fluctuations. Counterintuitively, spectral fluctuations are much stronger for a regular motion when the level positions are uncorrelated and have the Poisson distribution.

The nearest level spacing distribution that follows from eq. (87) is the first (and the weakest) feature of quantum chaos, in many-body systems it is reached already at a small interaction strength [168. More sensitive measures deal with the fluctuations of the level number along large fragments of the spectrum [173]. Such an analysis is useful for checking the completeness and purity of long empirical level sequences, for example for low-energy neutron resonances which are important in practical applications [174, 175, 176]. For the purpose of this review, we have to emphasize that such statistical applications assume that the long-lived resonances can be considered as representatives for the sequences of stationary states although in reality they belong to the continuum.

The most interesting and far-reaching predictions of many-body quantum chaos refer to the structure of stationary states in the chaotic regime. Strongly mixed states in a narrow energy window have essentially the same degree of complexity [177] and the same macroscopic properties corresponding to thermodynamic equilibrium which provides a modern justification of statistical mechanics [166]. In the limit of random matrix theory, for large dimensions of the Hamiltonian matrices, the components of the chaotic wave functions are uncorrelated Gaussian variables. Consider, for example, an experiment that singles out a certain component of the wave function. Probing various states of similar structure, one would then discover that the distribution of corresponding probabilities is the chi-square for one degree of freedom, called the Porter-Thomas distribution (PTD) in nuclear spectroscopy [178]. The old experiments for neutron resonances confirmed this prediction. The joint analysis of $\nu>1$ components, in a similar way, is expected to reveal the chi-square distribution for $\nu$ degrees of freedom.

The closer study of chaotic wave functions shows [179] that small perturbations acting in this region of states turn out to be enhanced compared to their effects for regular wave functions of simple configurations. This follows from the fact that, on the road to chaos, the matrix elements of simple operators between chaotic functions of a similar degree of complexity decrease slower than the energy denominators in perturbation theory for the high level density. This chaotic enhancement is responsible for the unusually high, up to $10 \%$, level of the parity violation effects in interactions of slow neutrons with heavy nuclei [180, 181. The chaotic mechanism of the enhancement is confirmed by the experiments on parity violation in nuclear fission by longitudinally polarized neutrons [182]. The significant asymmetry of heavy fragments with respect to the neutron spin does not depend on the final distributions of product masses and kinetic energies being predetermined in the chaotic ("hot") stage of the compound nucleus. 
Among other practically important applications of the ideas of quantum chaos we can mention the extraction of information about invisible fine structure states in experiments with insufficient resolution [183. New computational methods for large-scale diagonalization of Hamiltonian matrices of extremely high dimension are based on the chaotic properties of highly excited configurations [184, 185, and resulting exponential convergence of the quantities of spectroscopic interest - low-lying energies and other observables - as a function of progressive truncation of the matrix preordered in a specific way.

\subsection{Statistics of resonances}

Many experimentally known sequences of complicated quantum states with identical global symmetry in fact are resonances observed in the continuum spectrum and therefore having finite lifetime. A natural question is if the properties of quantum chaos predicted by the random matrix theory for closed systems are still valid for quasistationary states.

Quantum theory of resonance states in complex systems is mostly developed for two limits clearly distinguished, for example, in neutron reactions on heavy targets. Not very far from thresholds, the neutron resonances appear as quite narrow well isolated peaks in neutron cross sections. Each resonance here corresponds to a long-lived compound state probed in a specific mode of its excitation (entrance channel) and decay (exit channel). In elastic neutron scattering, the experiment singles out a certain component of the quasistationary wave function, namely the one corresponding to the continuum neutron (typically in $s$-wave at low energies) and the ground state of the target. Here the resonance neutron widths, being proportional to the weight of this component in the compound state, are expected to obey the PTD, as for genuinely stationary states. In the opposite limit of broad resonances, their widths exceed the energy spacings. The cross sections in the region of overlapping resonances manifest a complex picture of wild interference (Ericson fluctuations [43, 44]) with the correlational length of cross sections that is usually assumed to be determined by the average width of overlapping resonances. The latter quantity is defined by the inverse mean lifetime of complicated intrinsic states. The deviations from this limiting picture are known experimentally for a long time, see for example [96, 186, 28 and references therein. The extreme statistical averaging which is a core of the Ericson theory does not account for unitarity requirements which imply collective super-radiance and trapping phenomena occuring even if internal dynamics is chaotic.

In the no-overlap regime of resonances in a chaotic many-body system, one can still expect the statistics of resonance parameters (energies and widths) to be in approximate agreement with the predictions of random matrix theory formulated for stationary states. This opinion was widely accepted but the recent accumulation of experimental data of higher precision [187, [188 put this statement in doubt. Indeed, we mentioned, eq. (88), the stationary level repulsion at small distances. However, this is a property of a mixing by a Hermitian interaction. For unstable states, their energies are defined within uncertainty 
related to their lifetime $\tau \sim \hbar / \Gamma$. There is no reason to expect the repulsion of resonances at short distances in energy comparable with their widths. The exact description in terms of the effective non-Hermitian Hamiltonian confirms the disappearance of such repulsion as can be seen already from our two-level model above, Sec. 4.1. Fig. 6 taken from Ref. [189], where the case of a single open channel was studied, shows that, as a function of the increasing continuum coupling constant $\kappa$, the probability $P(0)$ to have coinciding or very close positions of resonances on a real energy axis (level crossing) is growing from zero and falls down again after the super-radiant transition when the broad state spreads into a part of the background. The dependence on the number of open channels and strength of the intrinsic interaction is illustrated by Fig. 7 [47. In the GOE case all curves are qualitatively similar but the effect is getting more pronounced with the number of channels increasing. The model with twobody random interactions (TBRE), see also Section 7.4, shows that after the super-radiant transition $P(0)$ falls down as a function of $\kappa$ in a universal way since, independently of the strength of the two-body interaction, we are dealing with uniformly trapped states.

Moreover, the same qualitative argument of the preceding paragraph predicts also that energy centroids and widths of resonances cannot be independent, they have to be correlated, - the phenomenon clearly outside the standard theory. We need to construct a new statistical ensemble for unstable states generalizing the canonical Gaussian ensembles for closed systems. The similar ensemble of complex matrices 190 cannot serve as an appropriate model since its eigenvalues are spread over the complex plane while the physical widths correspond to the negative imaginary parts of the poles of the scattering matrix - resonance widths $\Gamma_{r}$ are positive. This property is guaranteed by the construction of the effective Hamiltonian $\mathcal{H}$ [the choice of $E^{(+)}$in eq. (24)]. The simplest appropriate statistical ensemble for unstable states (our $\mathcal{Q}$-space) assumes the intrinsic GOE dynamics $\left(H_{\mathcal{Q}}\right.$ part) along with uncorrelated decay amplitudes (parameters $A_{1}^{c}$ of the coupling Hamiltonian $H_{\mathcal{P} \mathcal{Q}}$ ). These amplitudes include kinematic factors depending on energy and vanishing at thresholds. As mentioned in our discussion of the continuum shell model, the energy dependence is crucial in realistic shell model studies. Dividing out this dependence we come to the reduced widths which can be considered as statistically independent. The PTD was expected to work just for these reduced quantities.

Assuming the statistical independence and similarity of all decay amplitudes one can take them as random Gaussian quantities with zero mean and variance

$$
\overline{A_{1}^{c} A_{2}^{c^{\prime}}}=\delta_{12} \delta^{c c^{\prime}} \frac{\gamma^{c}}{N},
$$

where $N$ is a large dimension of the $\mathcal{Q}$-space, and $\gamma^{c}$ parameterize characteristic partial widths. The ansatz $(89)$ is natural for the system with chaotic internal dynamics. However, one can argue that, in realistic applications, the assumption 89 can be reasonable, independently of the presence of internal chaos, like in the case of weakly chaotic or even regular intrinsic dynamics, for $N \gg 1$. In self-bound rotationally invariant systems, such as a heavy nucleus, 
we need to consider the states with the same quantum numbers $J, M$ of angular momentum (plus parity and isospin). For a given shell-model partition, there are many ways of angular momentum coupling leading to the same total symmetry. These paths expressed through the fractional parentage coefficients are essentially chaotic. The idea of random walk in the angular momentum space was used long ago by Bethe [191] for estimates of the spin-dependent nuclear level density. This geometric chaoticity plays an important role in statistics of self-bound systems [192, 184, 185, 193] and it can justify the assumption (89) even for the case of intrinsic interactions which are relatively weak to introduce the full dynamical chaos. Irrespectively of dynamic interaction, this leads to chaotic structure of wave functions and, for example, to the predominance of the scalar representation $J=0$ in the ground state of an even-even nucleus after averaging over all interactions allowed by the conservation laws [194, 195].

With the GOE intrinsic dynamics and a single decay channel, it was derived algebraically [9, 10] that the common distribution function of complex energies (6) is given by the Ullah distribution [196],

$$
P\left(\mathcal{E}_{1}, \ldots, \mathcal{E}_{N}\right)=C_{N}\left(\prod_{n} \frac{1}{\sqrt{\Gamma_{n}}}\right)\left(\prod_{m<n} \frac{\left|\mathcal{E}_{m}-\mathcal{E}_{n}\right|^{2}}{\left|\mathcal{E}_{m}-\mathcal{E}_{n}^{*}\right|}\right) \exp \left[-N F\left(E_{n}, \Gamma_{n}\right)\right],
$$

where the "free energy" of interacting resonances is given by

$$
F=\frac{1}{a^{2}} \sum_{n} E_{n}^{2}+\frac{1}{\gamma} \sum_{n} \Gamma_{n}+\frac{1}{2 a^{2}} \sum_{m<n} \Gamma_{m} \Gamma_{n}
$$

The distribution in the complex energy plane 90 reflects the interplay of various dynamical factors. The product of inverse square roots of the widths is the remnant of the PTD that is produced by the transformation from the amplitudes of given components in a complicated superposition of wave functions to their probabilities. The next product has the numerator responsible for the quadratic repulsion of energies in the complex plane, similar to the Gaussian unitary ensemble because the openness of the system effectively works as violation of time-reversal invariance. The denominator in this product shows "attraction" of the widths to their "electrostatic images" and therefore the accumulation of the widths in the vicinity of the real axis (many long-lived states). The real axis remains a singular boundary that excludes the appearance of negative widths. We can recall that the electrostatic analogy was fruitful in the first studies of quantum chaos by Dyson [197. The free energy (91) is the main factor regulating the equilibrium arrangement of the complex energies. The super-radiance is contained in the last sum (repulsive width interaction). When typical widths grow beyond average spacings of real energies, the last term in $F$ suppresses the probabilities of all configurations with uniform width distribution. The prominent maximum of probability is realized then on the configuration with one predominant width (super-radiant state). This means that the effect of super-radiance may coexist with chaotic internal dynamics.

In many-channel situations, direct algebraic derivation of the joint distribution function for complex energies is difficult and the more advanced methods 
related to supersymmetry should be applied. Such methods were developed first for closed systems [198] and then applied to unstable systems as well [199]. Unfortunately, the results are typically expressed in the form of cumbersome multi-dimensional integrals that makes the analysis not an easy task (the calculations are usually simpler for the intrinsic part described by the GUE rather than GOE). In this situation the direct numerical simulation of statistical ensembles becomes the method of choice. We have seen in Fig. 2 [1] the evolution of the distribution of complex energies as the strength of continuum coupling grows. The effect of super-radiance is clearly revealed as the segregation of super-radiant states (in the amount equal to the number of open channels) from the larger cloud of trapped states. This transformation is rather sharp and reminds a phase transition. Regrettably, the experimental data for multiresonance systems with adjustable parameters that would allow to trace this restructuring are practically absent. The best candidates for this purpose are probably experiments with superconducting microwave cavities 163 and with acoustic chaos 200.

\subsection{Statistics of resonance widths}

The statistical distribution of the resonance widths is an essential characteristic of a sufficiently long sequence of resonances. The low-energy neutron scattering off heavy nuclei provides rich statistics of such data. Usually the primary treatment of the data in the regime of well separated resonances is accomplished with the aid of the $R$-matrix analysis 63 , which we do not discuss here. At energies below the first inelastic threshold, neutron scattering can be only elastic, and we can apply the one-channel consideration. We will also omit a discussion of extracting the energy dependence that leads to the reduced widths. In practice this procedure should depend on the specific features of the compound system since the standard energy dependence defined by the orbital momentum of the neutron may be distorted by the shape of the single-particle strength function in the closed system [69]. Assuming that we can have a reliable set of reduced resonance parameters, we can apply the statistical measures.

For the set of physically equivalent reduced widths of a single-channel reaction one could expect the PTD. The admixture of intruder resonances corresponding to different quantum numbers, such as $p$-wave resonances in the predominantly $s$-wave sequences, should violate the level spacing distribution and statistics of level fluctuations. Such an impurity supposedly will move the distribution in the direction of the two-channel situation, and therefore increase the effective number of degrees of freedom given by the parameter $\nu$ in the chi-square distribution,

$$
P_{\nu}(\gamma)=C_{\nu} \gamma^{\nu / 2-1} e^{-\gamma / \bar{\gamma}}
$$

Here we can notice that even for well studied cases, such as ${ }^{235} \mathrm{U}$, the undetected admixture of $p$-wave can reach few percent [175. Also we select an even-even target, so that all $s$-wave resonances have the same spin $1 / 2$. For many years, 
based on experimental data, it was thought that the PTD, eq. (92) with $\nu=1$, describes well the reduced neutron width distributions. However, recent precise measurements [187, 188] claim that there are statistically reliable deviations from the PTD in the direction of the effective parameter $\nu$ smaller than one.

The proper inclusion of the effects of coupling to continuum [201] with the use of the effective non-Hermitian Hamiltonian shows that such deviations inevitably appear even for the sequence of statistically equivalent resonances although complementary effects of single-particle strength functions [69] can also be important. The PTD corresponds to the complete factorization of the joint distribution (87) into the independent GOE-like distribution of the resonance centroids $E_{n}$ and the distribution of non-interacting widths. As seen from (86) this occurs only in the extreme limit of vanishingly small widths $\Gamma_{n}$ in comparison to the energy spacing $D \sim a / N$. The energy-width correlations enter the game and lead to the deviations from the PTD already at rather small value of the coupling strength $\kappa \sim \gamma / D$. The evolution of the width distribution as a function of increasing continuum coupling is illustrated by Fig. 8 [201]. Very soon, the chi-square model itself becomes a very bad approximation to the distribution predicted by $(90)$. In agreement with the random matrix results [202, 203, the tail of the distributions behaves $\propto(\Gamma / \bar{\Gamma})^{-2}$ that cannot be described by chi-square distributions.

\subsection{Role of intrinsic dynamics}

Another interesting aspect of the width distribution problem is related to the intrinsic dynamics. The exact results cited above all refer to the canonical Gaussian ensembles of random matrices. In such cases of developed quantum chaos, all intrinsic states are of the same degree of complexity and their uncorrelated components give rise to Gaussian distributions, essentially as a manifestation of the central limit theorem. A slightly different type of statistics arises if the interparticle interactions, being still random, carry certain selection rules. We have already mentioned the role of geometric chaoticity in the process leading to complicated mixing of the nuclear wave functions even in the absence of interactions.

Two-body interactions being the most relevant part of intrinsic dynamics bring a regular element in the Hamiltonian matrix of a closed system: many zeros and many repeated substructures 204. Therefore, even if the resulting local pattern of the level repulsion and spacing distribution is in this case similar to the GOE limit, the remaining correlations may have their influence upon the fine features, including the continuum coupling. This can be modeled with simulating the intrinsic Hamiltonian $H_{\mathcal{Q} \mathcal{Q}}$ by the two-body random ensemble (TBRE). Here the two-body matrix elements are uncorrelated random quantities with zero mean and variance $v^{2}$. The ratio $v / d$ of the TBRE strength to the appropriate mean-field level spacing characterizes the relative strength of intrinsic interactions. The estimates of the critical strength for the onset of chaos in the Fermi system of shell-model type with a certain number of particles in a truncated orbital space were given in Ref. [205]. 
Fig. 8 shows the best value for the chi-square parameter $\nu$ for different values of the continuum coupling strength $\kappa$ found 201 in the simulation of the dynamics by the GOE and TBRE with and without strong intrinsic interaction. The deviations from the chi-square distribution grow substantially faster for more regular dynamics both for one and two open channels. Intrinsic chaos is accompanied by the level repulsion and their relative ordering. Absence of chaos implies a stronger sensitivity to the continuum coupling. This physics sets the limits for the applicability of the traditional interpretation of the width of a neutron resonance as a measure for the strength of a pure neutron component in the wave function of compound nucleus. At the same time, we can acquire an instrument of evaluating the degree of intrinsic chaos by the observable width distributions for sufficiently clean resonance sequences. In general, various studies allows one to conclude that increasing the intrinsic interaction we suppress the fluctuations in the continuum making the compound states more uniform. 


\section{Signal transmission through a mesoscopic sys- tem}

\subsection{Common physics of mesoscopic systems}

Scattering of an external beam off a many-body system is the most powerful instrument for obtaining the indispensable information on the internal structure of the system. At the same time, the situation can be inverted and our goal might be just the propagation of the signal itself [206]. The signal can be not only transmitted - we may need to transform it, split and recombine, separate resonance components, selectively amplify or suppress parts of the signal etc. It might be important to implement such different tasks simultaneously and record the results. All these tasks are necessary for quantum computing and in

all modern applications of information treatment. A parallel problem is that of finding or synthesizing the quantum mesoscopic medium especially appropriate for a specific purpose.

In fact, all these problems can be addressed in the framework of the effective non-Hermitian Hamiltonian. Here we have the intrinsic system with possible resonances along with open exit and entrance channels, plus some channels closed at a certain energy (frequency) of the signal but virtually influencing the results of a process. A system can also be embedded into complex environment and subject to external noises and decoherence but this set of problems is beyond the scope of the current review although it can be analyzed with similar tools.

The terminology borrowed from nuclear reactions as a fully developed subfield can be successfully used for other mesoscopic systems. In all cases of the signal transmission we are interested in the exit-entrance relation given by the scattering $S$-matrix in the space of open channels. This matrix describes the probabilistic connection between the in- and out- quantum states. Without restriction of generality, this unitary matrix is defined by eq. (38), where we will be interested mainly in the second part that describes the signal propagation inside the system (compound states). The observable quantities are cross sections, or transmission probabilities, their correlations and fluctuations as functions of energy and intrinsic parameters of the system.

\subsection{Statistics of cross sections}

If the signal lives inside the system for sufficiently long time (longer than the Weisskopf time $\hbar / D$, where $1 / D$ is the typical density of states coupled to the signal), the situation becomes similar to the compound nucleus. In the experiment with good resolution we expect the cross section to reveal isolated narrow resonances corresponding to the levels of the compound system. As lifetime of intrinsic states shortens, the resonances broaden and start to overlap. Here one typically expects strong fluctuations of observable cross sections or transmission. The classical Ericson theory for the region of overlapping resonances [43, 44 does not analyze the properties of individual resonances, concentrating instead on the observable statistical features. However, this theory does not ac- 
count for the super-radiance effects since the coupling of intrinsic states through the continuum is not included into consideration.

If we neglect direct reactions, the $\hat{S}$-matrix can be presented in an explicitly unitary form (38),

$$
\hat{S}=1-i \hat{T}=\frac{1-(i / 2) \hat{K}}{1+(i / 2) \hat{K}},
$$

where the $K$-matrix (35) is given by the Green function of intrinsic propagation sandwiched between the continuum amplitudes,

$$
\hat{K}=\mathbf{A} \frac{1}{E-H} \mathbf{A}^{T} .
$$

The scattering characteristics can be subdivided into average and fluctuational parts 207. For example, it is assumed for the scattering amplitude that

$$
T^{a b}(E)=\overline{T^{a b}(E)}+T_{\mathrm{fl}}^{a b}(E),
$$

where the averaging is performed over various resonances or, in practice, over an energy interval including sufficiently many resonances of supposedly similar nature. The same separation of contributions is performed for the $S$-matrix and cross sections.

In the absence of direct reactions avoiding formation of the compound system, only elastic channels contribute to the average amplitude, $\overline{T^{a b}(E)} \propto \delta^{a b}$. In the language of the effective Hamiltonian, this is equivalent to the discussed above statistical ansatz (89) for the coupling amplitudes. Under this assumption, the averaging of the $K$-matrix gives 46 .

$$
\overline{K^{a b}(E)}=-i \delta^{a b} \kappa^{a}, \quad \kappa^{a}=\frac{\pi \gamma^{a}}{N D},
$$

where the density of states $\rho=1 / D$ should be taken in the center of the averaging energy interval containing $N$ resonances. At $N \gg 1$, this leads to the diagonal average $\hat{S}$-matrix,

$$
\overline{S^{a b}}=\delta^{a b} \frac{1-\kappa^{a}}{1+\kappa^{a}} .
$$

Assuming equivalent channels, $\kappa^{a}=\kappa$, we can express the total cross section with the aid of the optical theorem,

$$
\overline{\sigma_{\mathrm{tot}}}=\sum_{b} \overline{\sigma^{b a}}=2(1-\operatorname{Re} \bar{S})=\frac{4 \kappa}{1+\kappa},
$$

where we omit the prefactor $4 \pi / k^{2}$.

It is convenient to work with the transmission coefficient for each channel,

$$
\tau^{a}=1-\left|\overline{S^{a a}}\right|^{2}=\frac{4 \kappa^{a}}{\left(1+\kappa^{a}\right)^{2}} .
$$


According to the definition (96), this quantity, as well the average characteristics like $(98)$, does not depend on the intrinsic dynamics if the parameter $\kappa$ is always renormalized for the proper level density. The so-called perfect coupling regime in channel $a, \tau^{a}=1$, corresponds to $\kappa^{a}=1$ when the average $\hat{S}$-matrix $(96)$ vanishes. This is precisely our region of the super-radiant transition. We can note that the transmission $(98)$ is symmetric with respect to the substitution $\kappa \rightarrow 1 / \kappa$; in the language of super-radiance this means that at large $\kappa$ one of $N \gg 1$ resonances broadens and disappears, and the transmission pattern in a given channel returns to the regime of no overlap.

Similarly, the behavior of the fluctuating cross sections in various (by assumption, equivalent) channels can be encoded into another important parameter, the elastic enhancement factor, the ratio

$$
F=\frac{\overline{\sigma_{\mathrm{fl}}^{a a}}}{\overline{\sigma_{\mathrm{fl}}^{b a}}}, \quad b \neq a,
$$

of elastic to inelastic cross sections 208. In terms of the transmission coefficient and the elastic enhancement factor, we can derive

$$
\overline{\sigma^{\mathrm{el}}}=F \overline{\sigma^{\mathrm{inel}}}=\frac{F \tau}{F+M-1} .
$$

The conventional Hauser-Feshbach parametrization with $\overline{\sigma^{a b}} \propto\left(1+\delta^{a b}\right)$ leads to $F=2$. This result was repeatedly derived in various approaches [209, 199, 210] in the regime of strong intrinsic chaos. The numerical analysis 46] shows that the elastic enhancement factor goes down from $F \approx 3$ to $F=2$ with increase of the interaction strength reaching the standard value $F=2$ in the GOE limit. This behavior requires more detailed understanding. Fig. 9 from Ref. 46] shows the average elastic fluctuational cross section in the TBRE simulation close to the critical coupling regime, $\kappa=0.8$, as a function of the intrinsic TBRE interaction strength $\lambda=v / d$. We see the sharp transition to the GOE limit at the same value of $\lambda$ that corresponds to onset of chaos in the closed system.

\subsection{Fluctuations of cross sections}

It is accepted by the standard Ericson theory that in the overlapping regime the variance of the width distribution is small compared to the average width. Essentially this is based on the idea of partial widths being independent random variables. From our consideration of super-radiance it is clear that there is no room for Ericson fluctuations in the one-channel case because of a sharp transition from isolated resonances to the situation of one broad and many trapped states avoiding the overlapping regime. For many channels, $M \gg 1$, one could expect that the partial widths are independent random variables, so that both, the variance $\operatorname{Var}(\Gamma)$ and the average total width $\bar{\Gamma}$, grow linearly with $M$. Then the ratio $g=\operatorname{Var}(\Gamma) / \bar{\Gamma}^{2}$ falls down as $1 / M$. We have discussed earlier the width distribution for one open channel at realistic (small for the neutron 
resonances) values of $\kappa$. At $\kappa \sim 1$, the ratio $g$ is not small and strongly depends on $\kappa$. As a function of $M$, Fig. 10, it falls much slower than $1 / M$ revealing a trend to saturation; one can clearly see the dependence on intrinsic dynamics (parameter $\lambda$ ) that is getting stronger as $\kappa$ grows. Again the onset of intrinsic chaos is smoothing the width fluctuations.

In the case of intrinsic dynamics described by the GOE, the simulations agree with the prediction of a singularity at the critical coupling,

$$
g=\frac{\operatorname{Var}(\Gamma)}{\bar{\Gamma}^{2}} \propto \frac{\ln ^{2}(1-\kappa)}{(1-\kappa)^{2}} .
$$

This singularity is revealed, for any value of $\lambda$, in the TBRE case as well. Another characteristic feature is the presence of correlations between the widths and the fluctuating part of the resonance amplitudes of the $S$-matrix. Such correlations are amplified in the region of critical coupling.

The conventional theory of overlapping resonances predicts that the correlation function of cross sections,

$$
C_{\sigma}(\epsilon)=\overline{\sigma(E) \sigma(E+\epsilon)}-\overline{\sigma(E)}^{2},
$$

as well as the similar correlation function $C_{T}(\epsilon)$ for the scattering amplitude are essentially the same. Being expressed as ratios $r(\epsilon)=C(\epsilon) / C(0)$, they have a simple form

$$
r(\epsilon)=\frac{l^{2}}{\epsilon^{2}+l^{2}},
$$

and the correlation lengths coincide, $l_{\sigma}=l_{T}$, being equal to the average width $\bar{\Gamma}$. The qualitative argument in favor of this identification uses the idea that it is possible to keep correlations only on a time scale of the mean lifetime of the resonances, $\sim \hbar / \bar{\Gamma}$. Such arguments do not account for the effects of super-radiance. The analysis [46] confirmed that the two correlation lengths are equal for large $M$, while $l_{\sigma}<l_{T}$ for small number of channels, when the Ericson assumption are invalid, especially for weak intrinsic interaction. The fluctuations are not reproducible in different channels of the reaction. The correlation function is also different from eq. (104), in agreement with Ref. 211. The equality $l=\bar{\Gamma}$ is not valid, except for the case of very small $\kappa$, as it was recognized long ago [186, 57, 212. Indeed, in the presence of the superradiant trend, there is no justification for considering these quantities identical. Instead, the correlation length at large $M$ agrees well with the expression [12] through the transmission coefficient,

$$
\frac{l}{D}=\frac{M \tau}{2 \pi}=\frac{M}{2 \pi} \frac{4 \kappa}{(1+\kappa)^{2}} .
$$

Here the Weisskopf time $\hbar / D$ plays the role of the characteristic unit to measure the intrinsic life of the signal inside the system. For the same level density $1 / D$, the results do not depend on the intrinsic interaction strength $\lambda$. The general 
relation between the average resonance width and the transmission coefficient is given by the Moldauer-Simmonius formula [213, 214,

$$
\frac{\bar{\Gamma}}{D}=-\frac{M}{2 \pi} \ln (1-\tau) .
$$

Only at small $\tau$ eqs. 105 and 106 lead to the equality $l=\bar{\Gamma}$.

\subsection{From Ericson to conductance fluctuations}

The so-called universal conductance fluctuations [215, 216, 42, 217] in electronic mesoscopic systems can be considered on the same footing as nuclear reactions; this analogy was repeatedly discussed in the literature [45, 46, 47. The analysis with the aid of the effective non-Hermitian Hamiltonian 48] demonstrates how even delicate predictions of theory of universal conductance fluctuations are reproduced by the consideration based on a general quantum-mechanical foundation.

The conductance of a mesoscopic system can be defined according to Landauer as [42] a sum of partial cross sections,

$$
G=\sum_{b=1}^{M / 2} \sum_{a=M / 2+1}^{M} \sigma^{a b} .
$$

In order to establish the direct connection with conductance theory, we divide here our $M$ channels, $M$ considered to be an even number, into two equal groups of entrance, $b$, and exit, $a$, channels (this limitation certainly can be eliminated, see for example [218); we also omit the standard dimensional factor $2 e^{2} / \hbar$. For equivalent channels, the average conductance can be directly expressed through the average cross section 100 and the elastic enhancement factor,

$$
\bar{G}=\left(\frac{M}{2}\right)^{2} \overline{\sigma^{a b}}=\frac{M^{2} \tau}{4(F+M-1)} .
$$

At $M \gg 1$, the limiting value $M \tau / 4$ is fully determined by the number of channels and the transmission $\tau$. The simulations 48 are in good agreement with this prediction both for intrinsic interactions described by the GOE and by the TBRE.

The fluctuations of conductance depend on the degree of chaos inside the system. The analytical results available for the GOE intrinsic dynamics [170, 206, 42 predict the variance $\operatorname{Var}(G)$ evolving between $2 / 15$ in the ballistic regime to $1 / 8$ in the diffusive regime. The approach of the effective Hamiltonian indeed reproduces these values, see Fig. 11 taken from [48. The crossover from one to another regime occurs precisely at the value of the intrinsic interaction parameter $\lambda$ that corresponds to the onset of chaos in the closed system decoupled from the continuum. This and more detailed results on $\operatorname{Var}(G)$, both for the GOE and for other models of internal dynamics, can be used for extracting 
information on the degree of chaos inside the system from the experimental conductance data, for example, as a function of the degree of openness of quantum dots. Especially useful might be results on the various correlations of "elastic" $(a=b)$ and "inelastic" $(a \neq b)$ cross sections; the correlations turn out to be negative for the processes without a common channel. As a function of the continuum coupling, the maximum sensitivity to the intrinsic chaos is observed at the intermediate strength of continuum coupling while at the perfect coupling the continuum effects overshadow the information coming from inside. An interesting object of application of these results can be found in correlations of speckle patterns [219, 220].

\subsection{Transport through nanostructures}

Without trying to give a full review of this extremely rapidly developing area, we only point out the relation of this tempestuous activity to the main topic of this article. In Section 2.2 we gave an example of the tight-binding model for onedimensional lattice serving as a quantum wire for transmission of a signal, in this case propagation of an electron through a sequence of potential wells coupled by tunneling among themselves and through end barriers to the external world. This simplified model discussed originally in Refs. [13] and [52] was recently generalized and put on a realistic foundation [49, 50]. The whole approach with the idea of super-radiance and trapping in an open many-body system described

by the effective non-Hermitian Hamiltonian was translated into the language of quantum dots in Ref. 221. This is a prototype of many practical arrangements including semiconductor superlattices and quantum dot arrays [222, 223, 224], molecular bridges 225, 226] etc. Similar physics turns out to be appropriate for the description of energy transfer in biological "antennae" (like photosynthetic bacteria [227]). The external barriers implement the controllable coupling to the continuum. It was shown 49 that a realistic sequence of potential barriers can be precisely modeled in the same approach and related to the open Anderson model 228] (the exact correspondence holds for weak coupling between the sites).

A general situation with asymmetric coupling, recall the parameters $\gamma_{L}$ and $\gamma_{R}$ in the Hamiltonian (7) and the solution for the resonances given by eqs. (12) and (13), was analyzed in detail in Ref. [50. Two realistically important cases are distinguished by the ordered character of the chain in contrast to the presence of internal disorder. In the case of a chain with $N \geq 2$ sites without disorder, the transport near the center of the energy band (8) reveals perfect transmission $\tau=1$ at all resonance peaks for the critical value, $\gamma_{\mathrm{cr}}=2 v \sqrt{q}$ of the entrance width, where $v$ is the tunneling amplitude, and we set $\gamma_{L}=\gamma, \gamma_{R}=$ $\gamma / q$ introducing the asymmetry parameter $q$. The same value $\gamma_{\text {cr }}$ provides the maximum of the transmission integrated over the energy band.

The transmission is determined by the sums $(13)$ over the Bloch states inside the energy band. These sums can be calculated [50] as functions of $\epsilon=E / 2 v$ 
in the closed form,

$$
P_{ \pm}(\epsilon)=(1 \pm 1) \frac{\epsilon}{4 v}-\frac{\epsilon^{2}-1}{v\left(z_{+}-z_{-}\right)}\left[\frac{z_{+}^{\alpha_{ \pm}}}{z_{+}^{2 N+2}-1}-\frac{z_{-}^{\alpha_{ \pm}}}{z_{-}^{2 N+2}-1}\right]
$$

where $z_{ \pm}=\epsilon \pm \sqrt{\epsilon^{2}-1}, \alpha_{+}=2 N+2$, and $\alpha_{-}=N+1$. Inside the band $\epsilon=\cos \beta$ and $z_{ \pm}=\exp ( \pm i \beta)$. This leads to the "elastic" transmission (in this formulation back scattering) expressed in terms of $\eta_{L . R}=\gamma_{L, R} / 2 v$,

$$
\tau^{L L}=4 \eta_{L}^{2} \frac{\eta_{L}\left(1+\eta_{L} \eta_{R}+\eta_{R}^{2}\right)+\eta_{R} \cos (2 \beta)+\left(1+\eta_{R}^{2}\right) \sin \beta}{\left(\eta_{L}+\eta_{R}\right)\left(1+\eta_{L} \eta_{R}\right)\left(1+\eta_{L}^{2}+2 \eta_{L} \sin \beta\right)},
$$

and analogously for $\tau^{R R}$. The "inelastic" channel (transmission) is characterized by

$$
\tau^{L R}=4 \eta_{L} \eta_{R} \frac{\sin \beta}{\left(\eta_{L}+\eta_{R}\right)\left(1+\eta_{L} \eta_{R}\right)},
$$

so that the condition of unitarity, $\tau^{L L}+\tau^{L R}=-2 \operatorname{Im} T^{L L}$ is fulfilled. The transmission integrated over the energy band of the width $4 v$ and shown in Fig. 12 is well described by the same equation that is valid for two intrinsic states,

$$
\frac{1}{4 v} \int d E \tau(E)=\frac{\pi \gamma}{2 v} \frac{1}{(q+1)\left[1+\gamma^{2} /\left(4 v^{2} q\right)\right]} .
$$

The transmission maximum corresponds to $\gamma=\gamma_{\mathrm{cr}}$, or $\gamma_{L} \gamma_{R}=4 v^{2}$, the point of the "balance" between decay outside and tunneling inside the chain. This condition agrees with the results of Ref. [229].

The super-radiant effect, as we have already mentioned in similar context above, survives the intrinsic chaotic dynamics, in this case the diagonal sitedisorder [52, 50]. For weak disorder, the maximum transmission occurs at the same critical values of the continuum coupling. In the case of strong disorder, with the width of site energy fluctuation exceeding the tunneling strength $v$, the maximum transmission is shifted to higher values of the ratio $\gamma / v$. The width average reveals two super-radiant transitions at the expected values of the continuum coupling, $\gamma=2 v$ and $\gamma=2 q v$. The details of this dynamics are discussed in ref. [50, along with the comparison to the one-dimensional Anderson model [42, 230, 231 with symmetric and asymmetric leads.

Coupled quantum dots have been already studied and used in the experiments directed to the development of quantum informatics [232, 233. The effects similar to what have been discussed in Sec. 4.1, including the decoupling of special states from the leads that is equivalent to the appearance of bound states embedded in the continuum were discovered in [234, 235]. There is a growing interest to the geometry more complicated than a simple one-dimensional chain [236, 237. A three-dimensional transport diagram was probed [238] in triple quantum dots, where the conditions for stable transport were established. In a similar direction the search was performed with $Y$-junctions of superconducting Josephson chains [239] and $T$-shaped quantum dot structures [240]. Another possible geometry corresponds to a tetrahedral qubit [241]. 
The theoretical results discussed above can be directly generalized to plane or three-dimensional networks of various structures [50]. It was already noticed [239] that the $Y$-junctions behave similarly to quantum Brownian motion on frustrated planar lattices. In the simplest models, continuum coupling is concentrated at the perimeter. This arrangement can model an array of quantum dots or a particle tunneling through a lattice, with or without local disorder. At strong disorder, again the maximum transmission corresponds to the coupling strength growing proportional to the degree of disorder. Especially interesting can be the study of crossed chains that could be elements of future quantum computers 242. The crossing point in such models plays a singular role connecting all legs of the scheme. In addition to the Bloch energy bands, here, even with no disorder, the states of different nature appear, namely quasilocalized states with energy outside of the band and with wave functions exponentially decaying from the central point (evanescent waves).

For the analysis of transport through realistic nanostructures it would be important to study, theoretically and experimentally, the influence of external noise (or "dephasing" 243]) in addition to internal disorder. One inevitable source of noise is provided by electromagnetic fluctuations in the chain and in the background, see for example [244, 245]. In our approach it can be modeled by adding many weak incoherent channels. Another extremely important features which should be introduced in the consideration are the effects of magnetic field. 


\section{Conclusion and outlook}

At the time of growing experimental and theoretical interest in open and marginally stable mesoscopic systems, it is useful to take a broad view of the whole field and find out the most general ideas and methods which would make possible to work out a more or less unified physical picture of various specific examples. The approach related to the effective non-Hermitian Hamiltonian is quite general and at the same time easily adaptable to different cases.

This approach, being in its starting formulation a rigorous application of well known quantum-mechanical ideas, keeps its generality and at the same time allows one to make proper approximations for concrete problems. The main new feature stressed by this approach is a collective interaction of internal states in a complex quantum system through their common continuum or radiation field. Being first suggested in quantum optics, this property turns out to be generic for all open quantum systems with many degrees of freedom. The process of such interactions can be traced all the way from almost independent well isolated resonances to super-radiance and trapping. Paradoxically, if the number of open channels does not increase too fast, the typical widths of narrow resonances become even narrower with excitation energy increasing. We tried to demonstrate the unified physical picture of such phenomena in various subfields and such different objects as nanostructures and exotic nuclei, complex atoms and hypothetical long-lived heavy particles. The nuclear Ericson fluctuations turn out to be almost identical to universal conductance fluctuations in quantum dots; there are suggestions to look for similar physics even in such classical events as seismic waves.

The unified formulation of the super-radiance physics of open systems allows physicists to understand better many questions which historically were asked and seemingly solved on different grounds and for different occasions. The segregation of broad and narrow resonances was first just an observation in complicated numerical simulations of nuclear reactions which seemed to be infinitely remote from coherent spontaneous radiation in the gas of two-level atoms. The details of Ericson fluctuations in the regime of overlapping resonances were not confirmed by the experiments - now we understand that the phenomenon of super-radiance (or perfect coupling to the leads in mesoscopic physics) related to the requirements of unitarity were not fully satisfied in the original formulation. We anticipate many such partial discoveries in further applications.

As for any alive physical theory, we still have many open questions which hopefully will be addressed in the future. Our shortest list would include

- clarification of the important problem of the role of internal chaos and interrelation between the regular or chaotic dynamics inside the system and its openness properties;

- role of the multitude of weak external channels present in many practical cases, decoherence and equilibrium in open mesoscopic systems; 
- better understanding of interplay between collectivity in intrinsic interactions, including superconducting correlations, and collectivity of superradiant type through the continuum;

- quantum signal transmission in complicated geometry;

- development of more powerful computational methods.

ACKNOWLEDGEMENTS. Some of the original ideas were worked out together with V.V. Sokolov. We are grateful to our coauthors on the latest stages of common work, G.P. Berman, G.L. Celardo, F.M. Izrailev, L. Kaplan, R.A. Sen'kov, A.M. Smith, and S. Sorathia for friendly and fruitful collaboration. A. Volya made a great contribution to the discussed ideas and their practical implementation. We acknowledge useful discussions with V.V. Flambaum, I. Rotter and H.A. Weidenmüller. The work was partially supported by the National Superconducting Cyclotron Laboratory at Michigan State University, NSF through the grants PHY-0244453, PHY-0555366 and PHY-0758099, and USA-Israel Binational Science Foundation. 


\section{References}

[1] R.H. Dicke, Phys. Rev. 93, 99 (1954).

[2] N. Skribanowitz, I.P. Herman, J.C. MacGillivray, and M.S. Feld, Phys. Rev. Lett. 30, 309 (1973).

[3] A.V. Andreev, V.I. Emel'yanov, and Yu. A. Il'inskiï, Cooperative Effects in Optics (IOP, Bristol, 1993).

[4] Super-radiance: Multiatomic Coherent Emission, ed. M.G. Benedict (Taylor and Francis, N.Y. 1996).

[5] L. Menshikov, Phys.-Usp. 42, 107 (1999).

[6] T. Watanabe, X.J. Wang, J.B. Murphy, J.Rose, Y. Shen, T. Tsang, L. Giannessi, P. Musumeci, and S. Reiche, Phys. Rev. Lett. 98, 034802 (2007).

[7] Coherent atomic matter waves. eds. R. Kaiser, C. Westbrook, and F. David, NATO Advanced Study Institute (Springer, Berlin, 2001).

[8] P. Kleinwächter and I. Rotter, Phys. Rev. C 32, 1742 (1985).

[9] V.V. Sokolov and V.G. Zelevinsky, Phys. Lett. B 202, 10 (1988).

[10] V.V. Sokolov and V.G. Zelevinsky, Nucl. Phys. A504, 562 (1989).

[11] F.M. Izrailev, D. Saher, and V.V. Sokolov, Phys. Rev. E 49, 130 (1994).

[12] N.Lehmann, D.Saher, V.V. Sokolov and H.-J. Sommers, Nucl. Phys. A582, 223 (1995).

[13] V.V. Sokolov and V.G. Zelevinsky, Ann. Phys. (N.Y.) 216, 323 (1992).

[14] H. Feshbach, Ann. Phys. (N.Y.) 5, 357 (1958).

[15] H. Feshbach, Ann. Phys. (N.Y.) 19, 287 (1962).

[16] U. Fano, Phys. Rev. 124, 1866 (1961).

[17] P.I. Kapur and R. Peierls, Proc. Roy. Soc. A166, 277 (1937).

[18] E.P. Wigner and L. Eisenbud, Phys. Rev. 72, 29 (1947).

[19] T. Teichmann and E.P. Wigner, Phys. Rev. 87, 123 (1952).

[20] E. Di Grezia and S. Esposito, Found. Phys. 38, 228 (2008).

[21] A.M. Lane and R.G. Thomas, Rev. Mod. Phys. 30, 257 (1958).

[22] H. Feshbach, Ann. Rev. Nucl. Sci. 8, 49 (1958).

[23] G. Breit, Theory of Resonance Reactions and Allied Topics, Handbuch der Physik, Band XVI/1 (Springer, Berlin, 1959). 
[24] M.S. Livšic, Sov. Phys. JETP, 4, 91 (1957).

[25] M.S. Livšic, Operators, oscillations, waves (open systems). Transl. Math. Monographs 34 (Americal Mathematical Society, Providence, 1973), translated from Russian edition (Nauka, Moscow, 1966); the biography of M.S. Livšic can be found in H. Dym, I. Gohberg, and N. Kravitsky, Operator Theory: Advances and Applications, 29, 6 (1988).

[26] C. Mahaux and H.A. Weidenmüller, Shell Model Approach to Nuclear Reactions (North Holland, Amsterdam, 1969).

[27] L. Durand, Phys. Rev. D 14, 3174 (1976).

[28] I. Rotter, Rep. Prog. Phys. 54, 635 (1991).

[29] A. Volya and V. Zelevinsky, Phys. Rev. C 67, 054322 (2003).

[30] A. Volya and V. Zelevinsky, Phys. Rev. Lett. 94052501 (2005).

[31] A. Volya and V. Zelevinsky, Phys. Rev. C 74, 064314 (2006).

[32] N. Michel, W. Nazarewicz, M. Ploszajczak, and J. Okolowicz, Phys. Rev. C 67, 054311 (2003).

[33] N. Michel, W. Nazarewicz, M. Ploszajczak, and T. Vertse, J. Phys. G 36, 013101 (2009).

[34] G. Garcia-Calderón, Adv. Quant. Chem. 60, 407 (2010).

[35] V.V. Flambaum, A.A. Gribakina, and G.F. Gribakin, Phys. Rev. A 54, 2066 (1996).

[36] V.B. Pavlov-Verevkin, Phys. Lett. A 129, 168 (1988).

[37] N. Auerbach, Phys. Rev. C 50, 1606 (1994).

[38] N. Auerbach and V. Zelevinsky, Phys. Rev. C 65, 034601 (2002).

[39] N. Auerbach, V. Zelevinsky, and A. Volya, Phys. Lett. B 590, 45 (2004).

[40] H. Feshbach, Rev. Mod. Phys. 46, 1 (1974).

[41] N. Auerbach and V. Zelevinsky, Nucl. Phys. A781, 67 (2007).

[42] C.W.J. Beenakker, Rev. Mod. Phys. 69, 731 (1997).

[43] T. Ericson, Ann. Phys. (N.Y.) 23, 390 (1963).

[44] T. Ericson and T. Mayer-Kuckuk, Ann. Rev. Nucl. Sci. 16, 183 (1966).

[45] H.A. Weidenmüller, Nucl. Phys. A518, 1 (1990).

[46] G.L. Celardo, F.M. Izrailev, V.G. Zelevinsky, and G.P. Berman, Phys. Rev. E 76, 031119 (2007). 
[47] G.L. Celardo, F.M. Izrailev, V.G. Zelevinsky, and G.P. Berman, Phys. Lett. B 659, 170 (2008).

[48] S. Sorathia, F.M. Izrailev, G.L. Celardo, V.G. Zelevinsky, and G.P. Berman, EPL 88, 27003 (2009).

[49] G.L. Celardo and L. Kaplan, Phys. Rev. B 79, 155108 (2010).

[50] G.L. Celardo, A.M. Smith, S. Sorathia, V.G. Zelevinsky, R.A. Sen'kov, and L. Kaplan, Phys. Rev. B 82, 165437 (2010).

[51] J. Schwinger, Quantum Mechanics (Springer, Berlin, 2001).

[52] A. Volya and V. Zelevinsky, in Nuclei and Mesoscopic Physics, ed. V. Zelevinsky (AIP Conference Proceedings 777, 2005) p. 229.

[53] R. Röhlsberger, K. Schlage, B. Sahoo, S. Couet, and R. Rüffer, Science 328, 1248 (2010).

[54] K. Beloy, BAPS 56, No. 2 (2011).

[55] J. Dukelsky, G.G. Dussel, C. Esebbag, and S. Pittel, Phys. Rev. Lett. 93, 050403 (2004).

[56] F. Pan, T. Wang, J. Pan, Y.-F. Li, and J.P. Draayer, Phys. Lett. A 341, $94(2005)$.

[57] T.A. Brody, J. Flores, J.B. French, P.A. Mello, A. Pandey, and S.S.M. Wong, Rev. Mod. Phys. 53, 385 (1981).

[58] C.E. Porter and R.G. Thomas, Phys. Rev. 104, 483 (1956).

[59] J.P. Jeukenne and C. Mahaux, Nucl. Phys. A136, 49 (1969).

[60] S. Karataglidis and K. Amos, Phys. Lett. B 660, 428 (2008).

[61] R.G. Thomas, Phys. Rev. 100, 25 (1955).

[62] C. Bloch, Nucl. Phys. 4, 503 (1957).

[63] P. Descouvemont and D. Baye, Rep. Prog. Phys. 73, 036301 (2010).

[64] C.A. Engelbrecht and H.A. Weidenmüller, Phys. Rev. C 8, 859 (1973).

[65] I.J. Thompson and F.M. Nunes, Nuclear Reactions for Astrophysics (Cambridge University Press, 2009).

[66] T.-Y. Wu and T. Ohmura, Quantum Theory of Scattering (Prentice Hall, New York, 1962).

[67] M.L. Goldberger and K.M. Watson, Collision Theory (John Wiley \& Sons, New York, 1964). 
[68] O.K. Rice, J. Chem. Phys. 1, 375 (1933).

[69] H.A. Weidenmüller, arXiv:1009.6099.

[70] V. Weisskopf, Phys. Rev. 52, 295 (1937).

[71] P.A. Moldauer, Phys. Rev. 171, 1164 (1968).

[72] I. Rotter, Phys. Rev. E 64, 036213 (2001).

[73] P. von Brentano, Phys. Rep. 264, 57 (1996).

[74] P. von Brentano and M. Philipp, Phys. Lett. B 454, 171 (1999).

[75] A. Magunov, I. Rotter, and S. Strakhova, J. Phys. B 32, 1669 (1999).

[76] A. Magunov, I. Rotter, and S. Strakhova, J. Phys. B 34, 29 (2001).

[77] P. von Brentano, Rev. Mex. Fiz. 40, 1 (2002).

[78] M. Philipp, P. von Brentano, G. Paskovici, and A. Richter, Phys. Rev. E 62, $1922(2000)$.

[79] P. von Brentano, R. Jolos, and H. Weidenmüller, Phys. Lett. B 534, 63 (2002).

[80] L.N. Cooper, Phys. Rev. 104, 1189 (1956).

[81] D. Brink and R.A. Broglia, Nuclear Superfluidity (Cambridge University Press, Cambridge, 2005).

[82] G. Potel, F. Barranco, E. Vigezzi, and R.A. Broglia, arXiv:0912.0847.

[83] E. Hernández, A. Mondragón, and A. Járegui, Rev. Mex. Fiz. 48, 6 (2002).

[84] H.M. Nussenzveig, Causality and Dispersion Relations (Academic Press, New York, 1972).

[85] V.G. Zelevinsky, Yad. Fiz. 72, 1 (2009).

[86] A. Volya, EPL 86, 52001 (2009).

[87] H.W. Barz, I. Rotter, and J. Höhn, Nucl. Phys. A275, 111 (1977); A307, 285 (1978).

[88] I. Rotter, Sov. J. Part. Nucl. 15, 341 (1984).

[89] B. Fladt, K.W. Schmid, and F. Grümmer, Ann. Phys. 184, 254, 300 (1988).

[90] E.P. Wigner, Phys. Rev. 73, 1002 (1948).

[91] A.I. Baz', Sov. Phys. JETP 33, 923 (1957).

[92] G. Breit, Phys. Rev. 107, 1612 (1957). 
[93] N. Ahsan and A. Volya, Phys. Rev. C 82, 064607 (2010).

[94] A. Volya, Phys. Rev. C 79, 044308 (2009).

[95] N. Michel, W. Nazarewicz, and M. Ploszajczak, Phys. Rev. C 75, 031301 (2007).

[96] G.E. Mitchell, in Quantum Chaos and Statistical Nuclear Physics, eds. T.H. Seligman and H. Nishioka (Lecture Notes in Physics, 263, Springer, Berlin, 1986) p. 55.

[97] K. Bennaceur, F. Novacki, J. Okolowicz, and M. Ploszajczak, Nucl. Phys. A651, 289 (1999).

[98] J. Okolowicz, M. Ploszajczak, and I. Rotter, Phys. Rep. 374, 271 (2003).

[99] B.A. Brown and B. Wildenthal, Ann. Rev. Nucl. Part. Sci. 38, 29 (1988).

[100] B.A. Brown, W. Richter, R. Julies, and B. Wildenthal, Ann. Phys. 182, 191 (1988).

[101] T. Ikegami and S. Iwata, J. Comp. Chem. 23, 310 (2002).

[102] C. Hoffman et al., Phys. Lett. B 672, 17 (2009).

[103] H. Feshbach, A.K. Kerman, and R.H. Lemmer, Ann. Phys. (N.Y.) 41, 230 (1967).

[104] N. Auerbach, J. Hüfner, A.K. Kerman, and C.M. Shakin, Rev. Mod. Phys. 44, 48 (1972).

[105] N. Auerbach and A. Klein, Nucl. Phys. A395, 77 (1983).

[106] P.F. Bortignon, A. Bracco, and R.A. Broglia, Giant Resonances: Nuclear Structure at Finite Temperature (Harwood Academic, Amsterdam, 1998).

[107] P. Adrich et al., Phys. Rev. Lett. 95, 132501 (2005).

[108] A.P. Tonchev et al., Phys. Rev. Lett. 104, 072501 (2010).

[109] V.V. Sokolov and V.G. Zelevinsky, Fizika (Zagreb) 22, 33 (1990).

[110] V. Zelevinsky and A. Volya, in Proceedings of 11th International Conference on Nuclear Reaction Mechanisms, ed. E. Gadioli, Varenna, 2006, p. 73.

[111] M. Hirata, J.H. Koch, F. Lenz, and E.J. Moniz, Ann. Phys. (N.Y.) 120, 205 (1979).

[112] S.K. Adhikari, Phys. Rev. C 27, 218 (1983).

[113] L.F. Canto, A. Romanelli, M.S. Hussein, and A.F.R. de Toledo Piza, Phys. Rev. Lett. 72, 2147 (1994). 
[114] B. Lauritzen, P.F. Bortignon, R.A. Broglia and V.G. Zelevinsky, Phys. Rev. Lett. 74, 5194 (1995).

[115] P. von Brentano, Z. Phys. A 306, 63 (1982).

[116] R. Melzer, P. von Brentano, and H.P.G. Schieck, Nucl. Phys. A432, 363 (1985).

[117] K.A. Snover, Nucl. Phys. A553, 153c (1993).

[118] H. Morinaga, Phys. Rev. 97, 444 (1955).

[119] D.H. Wilkinson, Phil. Mag. 1, 379 (1956).

[120] P.F. Bortignon, A. Bracco, D. Brink, and R.A. Broglia, Phys. Rev. Lett. 67, 3360 (1991).

[121] V.V. Sokolov and V. Zelevinsky, Phys. Rev. C 56, 311 (1997).

[122] P. Facchi and S. Pascazio, Phys. Rev. Lett. 89, 080401 (2002).

[123] P.G. Silvestrov, Phys. Rev. B 64, 113309 (2001).

[124] A. Amir, Y. Oreg, and Y. Imry, Phys. Rev. A 77, 050101(R) (2008).

[125] J.J. Gaardhøje, Ann. Rev. Nucl. Part. Sci. 42, 483 (1992).

[126] M.S. Hussein, V. Kharchenko, L.F. Canto, and R. Donangelo, Ann. Phys. (N.Y.) 284, 178 (2000).

[127] I. Kawata, H. Kono, Y. Fujimara, and A.D. Bandrauk, Phys. Rev. A 62, 031401(R) (2000).

[128] L.O. Baksmaty, C. Yannouleas, and U. Landman, Phys. Rev. Lett. 101, 136803 (2008).

[129] T. Laarman et al., Phys. Rev. Lett. 98, 058302 (2007).

[130] I.V. Hertel et al., Phys. Rev. Lett. 102, 023003 (2009).

[131] J. A. Franco-Villafane et al., to be published.

[132] H. Smith and C.J. Pethick, Bose-Einstein Condensation in Dilute Gases (Cambridge University Press, Cambridge, 2001).

[133] J.J. Griffin, Phys. Rev. Lett. 17, 478 (1966).

[134] H. Feshbach, A. Kerman, and S. Koonin, Ann. Phys. (N.Y.) 125, 429 (1980).

[135] U. Griesmann, N. Shen, J.P. Connerade, K. Sommer, and J. Hormes, J. Phys. B 21, L83 (1988). 
[136] J.P. Connerade, M.A. Baig, and M. Sweeney, J. Phys. B 23, 713 (1990).

[137] R. van Leeuwen, W. Ubachs and W. Hogervorst, J. Phys. B 27, 3891 (1994).

[138] E. Luc-Koenig, M. Aymar, R. van Leeuwen, W. Ubachs W, and W. Hogervorst, Phys. Rev. A 52, 208 (1995).

[139] M. Aymar, C.H. Greene, and E. Luc-Koenig, Rev. Mod. Phys. 68, 1015 (1996).

[140] H. Friedrich and D. Wintgen, Phys. Rev. A 31, 3964 (1985).

[141] H. Friedrich and D. Wintgen, Phys. Rev. A 32, 3231 (1985).

[142] B.L. Beers and L. Armstrong Jr. Phys. Rev. A 12, 2447 (1975).

[143] J. Horowitz and B.D. Serot, Nucl. Phys. A368, 503 (1981).

[144] N. Auerbach, A. Goldhaber, M.B. Johnson, M.D. Miller, and A. Picklesimer, Phys. Lett. B 182, 221 (1986).

[145] M. L'Huillier, N.V. Giai and N. Auerbach, Nucl. Phys. A519, 83 (1990).

[146] P. Bartsch et al., Eur. Phys. J. A4, 209 (1999).

[147] T. Nakano et al., Phys. Rev. Lett. 91, 012002 (2003).

[148] S. Stepanyan et al., Phys. Rev. Lett. 91, 252001 (2003).

[149] F. Iachello, in Workshop on $N^{*}$ Physics, eds. T.-S.H. Lee and W. Roberts (World Scientific, Singapore, 1997) p. 78.

[150] R. Jaffe and F. Wilczek, Phys. Rev. Lett. 91, 232003 (2003).

[151] M. Karliner and H.J. Lipkin, Phys. Lett. B586, 303 (2004).

[152] M. Battaglieri et al., Phys. Rev. Lett. 96, 042001 (2006).

[153] S.K. Choi et al., Phys. Rev. Lett. 91, 262001 (2003).

[154] D. Acosta et al., Phys. Rev. Lett. 93, 072001 (2004).

[155] V.M. Abazov et al., Phys. Rev. Lett. 93, 162002 (2004).

[156] S.K. Choi et al., Phys. Rev. Lett. 100, 142001 (2008).

[157] H.G. Schuster and W. Just, Deterministic Chaos, 4 ed.(Wiley-VCH,Berlin, 2005).

[158] M.C. Gutzwiller, Chaos in Classical and Quantum Mechanics (Springer, New York, 1990).

[159] F. Haake, Quantum Signatures of Chaos (Springer, New York, 1991). 
[160] M.L. Mehta, Random matrices, 3rd ed., (Elsevier/Academic Press, Amsterdam, 2004).

[161] D.L. Kaufman, I. Kosztin, and K. Schultena, Am. J. Phys. 67, 133 (1999).

[162] H.-J. Stöckmann, Quantum chaos: an introduction (Cambridge University Press, 2000).

[163] H. Rehfeld, C. Dembowski, H.-D. Gräf, A. Heine, R. Hofferbert and A. Richter, Physica E, 9, 518 (2001).

[164] G. Casati and B.V. Chirikov, eds. Quantum Chaos, Between Order and Disorder (Cambridge University Press, 1995).

[165] I.V. Lerner, B.L. Altshuelr and Y. Gefen, eds. Fundamental problems of mesoscopic physics: Interactions and decoherence, NATO Science Series, Vol. 154 (Kluwer Academic Publishers, Dordrecht, 2004).

[166] V. Zelevinsky, Ann. Rev. Nucl. Part. Sci., 46, 237 (1996).

[167] V.V. Flambaum, A.A. Gribakina, G.F. Gribakin, and M.G. Kozlov, Phys. Rev. A 50, 267 (1994).

[168] V. Zelevinsky, B.A. Brown, N. Frazier, and M. Horoi, Phys. Rep. 276, 85 (1996).

[169] C.E. Porter, in Statistical Theories of Spectra: Fluctuations, ed. by C. E. Porter (Academic, New York, 1965), p. 2.

[170] T. Guhr, A. Mueller-Groeling, and H.A. Weidenmüller, Phys. Rep. 299, 189 (1998).

[171] H.A. Weidenmüller and G.E. Mitchell, Rev. Mod. Phys. 81, 539 (2009).

[172] V. Zelevinsky, Quantum Physics, vol. 2 (Wiley-VCH, Weinheim, 2011).

[173] F.J. Dyson and M.L. Mehta, J. Math. Phys. 4, 701 (1963).

[174] U. Agvaanluvsan, G.E. Mitchell, J.F. Shriner Jr., and M.P. Pato, Nucl. Instr. Meth. A498, 459 (2003).

[175] D. Mulhall, Z. Huard, and V. Zelevinsky, Phys. Rev. C 76, 064611 (2007).

[176] D. Mulhall, Phys. Rev. C 80, 034612 (2009).

[177] I.C. Percival, J. Phys. B 6, L229 (1973).

[178] A. Bohr and B.R. Mottelson, Nuclear Structure (World Scientific, Singapore, 1998).

[179] O.P. Sushkov and V.V. Flambaum, Sov. Phys. Usp. 25, 1 (1982).

[180] N. Auerbach, Phys. Rev. C 45, R514 (1992). 
[181] J.D. Bowman, G.T. Garvey, M.B. Johnson, and G.E. Mitchell, Ann. Rev. Nucl. Part. Sci. 43, 829 (1993).

[182] A. Kotzle, P. Jesinger, F. Gnnenwein, G. A. Petrov, V. I. Petrova, A. M. Gagarsky, G. Danilyan, O. Zimmer and V. V. Nesvizhevsky. Nucl. Instr. Meth. A 440, 750 (2000).

[183] G. Kilgus, G. Kührer, S. Müller, A. Richter, and W. Knüpfer, Z. Phys. A326, 41 (1987).

[184] M. Horoi, A. Volya, and V. Zelevinsky, Phys. Rev. Lett. 82, 2064 (1999).

[185] M. Horoi, B.A. Brown, and V. Zelevinsky, Phys. Rev. C 67, 034363 (2003).

[186] E.P. Kanter, D. Kollewe, K. Komaki, I. Leuca, G.M. Temmer, and W.M. Gibson, Nucl. Phys. A319, 109 (1978).

[187] P. E. Koehler, F. Bečvař, M. Krtička, J.A. Harvey, and K.H. Guber, Phys. Rev. Lett. 105, 072502 (2010).

[188] E.S. Reich, Nature 466, 1034 (2010).

[189] S. Mizutori and V. Zelevinsky, Z. Phys. A346, 1 (1993).

[190] J. Ginibre, J. Math. Phys. 6, 440 (1965).

[191] H.A. Bethe, Rev. Mod. Phys. 9, 69 (1937).

[192] D. Mulhall, A. Volya, and V. Zelevinsky, Phys. Rev. Lett. 85, 4016 (2000).

[193] T. Papenbrock and H.A. Weidenmüller, Phys. Rev. C 73, 014311 (2006).

[194] C. Johnson, D. Dean, and G.F. Bertsch, Phys. Rev. Lett. 80, 2749 (1998).

[195] V. Zelevinsky and A. Volya, Phys. Rep. 391, 311 (2004).

[196] N. Ullah, J. Math. Phys, 10, 2099 (1969).

[197] F.J. Dyson, J. Math. Phys. 3, 140, 157, 166 (1962).

[198] K.B. Efetov, Supersymmetry in Disorder and Chaos (Cambridge University Press, 1997).

[199] J.J.M. Verbaarschot, H.A. Weidenmüller, and M.R. Zirnbauer, Phys. Rep. 129, 367 (1985).

[200] C. Ellegaard, K. Schaadt, and P. Bertelsen, Phys. Scripta, T90, 223 (2001).

[201] J.L. Celardo, N. Auerbach, F.M. Izrailev, and V. Zelevinsky, Phys. Rev. Lett. 106, 042501 (2011).

[202] Y.V. Fyodorov and H.-J. Sommers, J. Math. Phys. 38, 1918 (1997). 
[203] H.-J. Sommers, Y.V. Fyodorov, and M. Titov, J. Phys. A 32, L77 (1999).

[204] V.V. Flambaum and F.M. Izrailev, Phys. Rev. E 65, 13(R) (1997).

[205] V.V. Flambaum and F.M. Izrailev, Phys. Rev. E 56, 5144 (1997).

[206] P.A. Mello and N. Kumar, Quantum Transport in Mesoscopic Systems: Complexity and Statistical Fluctuations (Oxford University Press, 2004).

[207] F.L. Friedman and W.F. Weisskopf, in Niels Bohr and the Development of Physics, ed. W. Pauli (Pergamon Press, N.Y. 1955) p. 134.

[208] H.L. Harney, A. Richter, and H.A. Weidenmüller, Rev. Mod. Phys. 58, 607 (1986).

[209] D. Agassi, H.A. Weidenmüller, and G. Mantzouranis, Phys. Rep. 3, 145 (1975).

[210] J.J.M. Verbaarschot, Ann. Phys. 168, 368 (1986).

[211] F.M. Dittes, H.L. Harney, and A. Müller, Phys. Rev. A 45, 701 (1992).

[212] P.A. Moldauer, Phys. Rev. C 11, 426 (1975).

[213] P.A. Moldauer, Phys. Rev. 157, 907 (1967).

[214] M. Simmonius, Phys. Lett. 52, 279 (1974).

[215] B.L. Altshuler, Pis'ma Zh. Eksp. Teor. Fiz. 41, 530 (1985) [JETP Lett. 41, 648 (1985)].

[216] P. Lee and A. Stone, Phys. Rev. Lett. 55, 1622 (1985).

[217] Y. Imry and R. Landauer, Rev. Mod. Phys. 71, S306 (1999).

[218] L.C. Botten, A.A. Asatryan, N.A. Nicorovici, R.C. McPhedran, and C.M. Sterke, Physica B 394, 320 (2007).

[219] S. Feng, C. Kane, P.A. Lee, and A.D. Stone, Phys. Rev. Lett. 61, 834 (1988).

[220] I. Freud, M. Rosenbluth, and S. Feng, Phys. Rev. Lett. 61, 2328 (1988).

[221] R. Berkovits, F. von Oppen, and J.W. Kantelhardt, Europhys. Lett. 68, 699 (2004).

[222] R. Tsu and L. Esaki, Appl. Phys. Lett. 22, 562 (1973).

[223] C. Pacher, C. Rauch, G. Strasser, E. Gornik, F. Elsholz, A. Wacker, C. Kiesslich, and E. Schöll, Appl. Phys. Lett. 79, 1486 (2001).

[224] G.V. Morozov, D.W.L. Sprung, and J. Martorell, J. Phys. D 35, 2091, 3052 (2002). 
[225] D. Walter, D. Neuhauser, and R. Baer, Chem. Phys. 299, 139 (2004).

[226] S.K. Maiti, Chem. Phys. 331, 254 (2007).

[227] J. Ray and N. Makri, J. Phys. Chem. A 103, 9417 (1999).

[228] P.W. Anderson, Phys. Rev. 109, 1492 (1958).

[229] C. Pacher and E. Gornik, Phys. Rev. B 68, 155319 (2003).

[230] S. Ida, H.A. Weidenmüller, and J.A. Zuk, Phys. Rev. Lett. 64, 583 (1990); Ann. Phys. 200, 219 (1990).

[231] J.A. Melsen and C.W. J. Beenakker, Phys. Rev. B 51, 14483 (1995).

[232] R. Hanson, L.P. Kouwenhoven, J.R. Petta, S. Tarucha, and L.M.K. Vandersypen, Rev. Mod, Phys. 79, 1217 (2007).

[233] S. Kitavittaya, A. Rastelli, and O.G. Schmidt, Rep. Prog. Phys. 72, $046502(2009)$.

[234] M.L. Ladron de Guevara, F. Claro, and P.A. Orellana, Phys. Rev. B 67, 195335 (2003).

[235] M.L. Ladron de Guevara and P.A. Orellana, Phys. Rev. B 73, 205303 (2006).

[236] G.C. Solomon et al., J. Chem. Phys. 129, 054701 (2008).

[237] L.-Y. Hsu and B.-Y. Jin, Chem. Phys. 355, 177 (2009).

[238] G. Granger et al., Phys. Rev. B 82, 075304 (2010).

[239] D. Giuliano and P. Sodano, Nucl. Phys. B811, 395 (2009).

[240] L.F. Santos and M.I. Dykman, Phys. Rev. B 68, 214410 (2003).

[241] M.V. Feigel'man, L.B. Ioffe, V.B. Geshkenbein, P. Dayal, and G. Blatter, Phys. Rev. B 70, 224524 (2004).

[242] H. Yan et al., Nature 470, 240 (2011).

[243] A, Cresti, Ann. Phys. 323, 28881 (2008).

[244] H. Kohler, F. Guinea, F. Sols, Ann. Phys. 31, 127 (2004).

[245] B. Ischi, M. Hilke, and M. Dubé, Phys. Rev. B 71, 195325 (2005). 

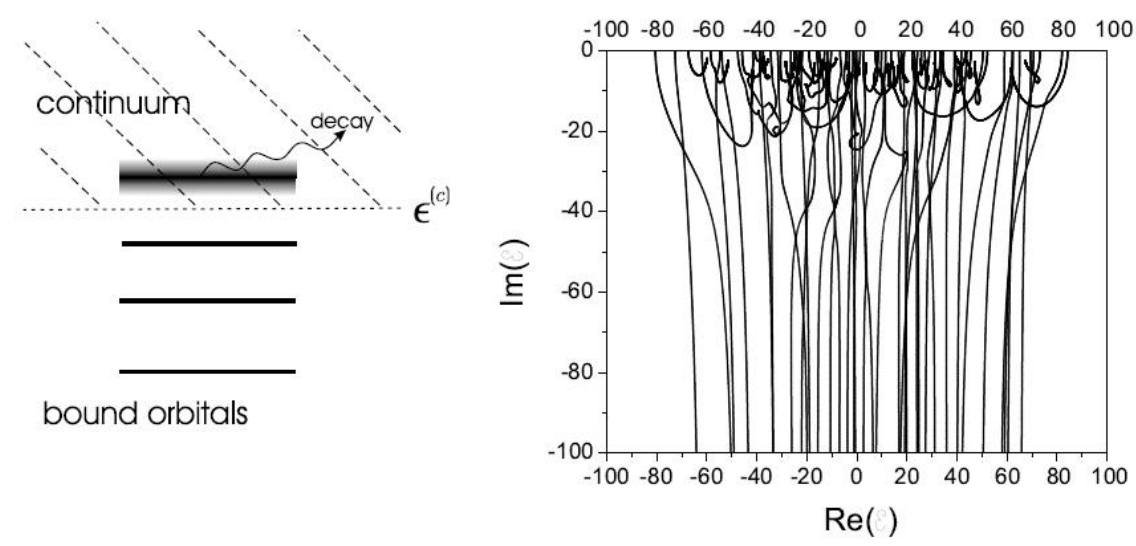

Figure 1: A system of 4 fermions in 8 single-particle levels [8!/(4!4!) = 70 manybody states] with the upper orbital in the continuum. Particles interact via random Hermitian interaction. The lines (right panel) show the trajectories of many-body energies in the complex plane as the width of the upper singleparticle level increases; $7 ! /(4 ! 3 !)$ many-body states become trapped and return to the vicinity of the real axis [52]. 

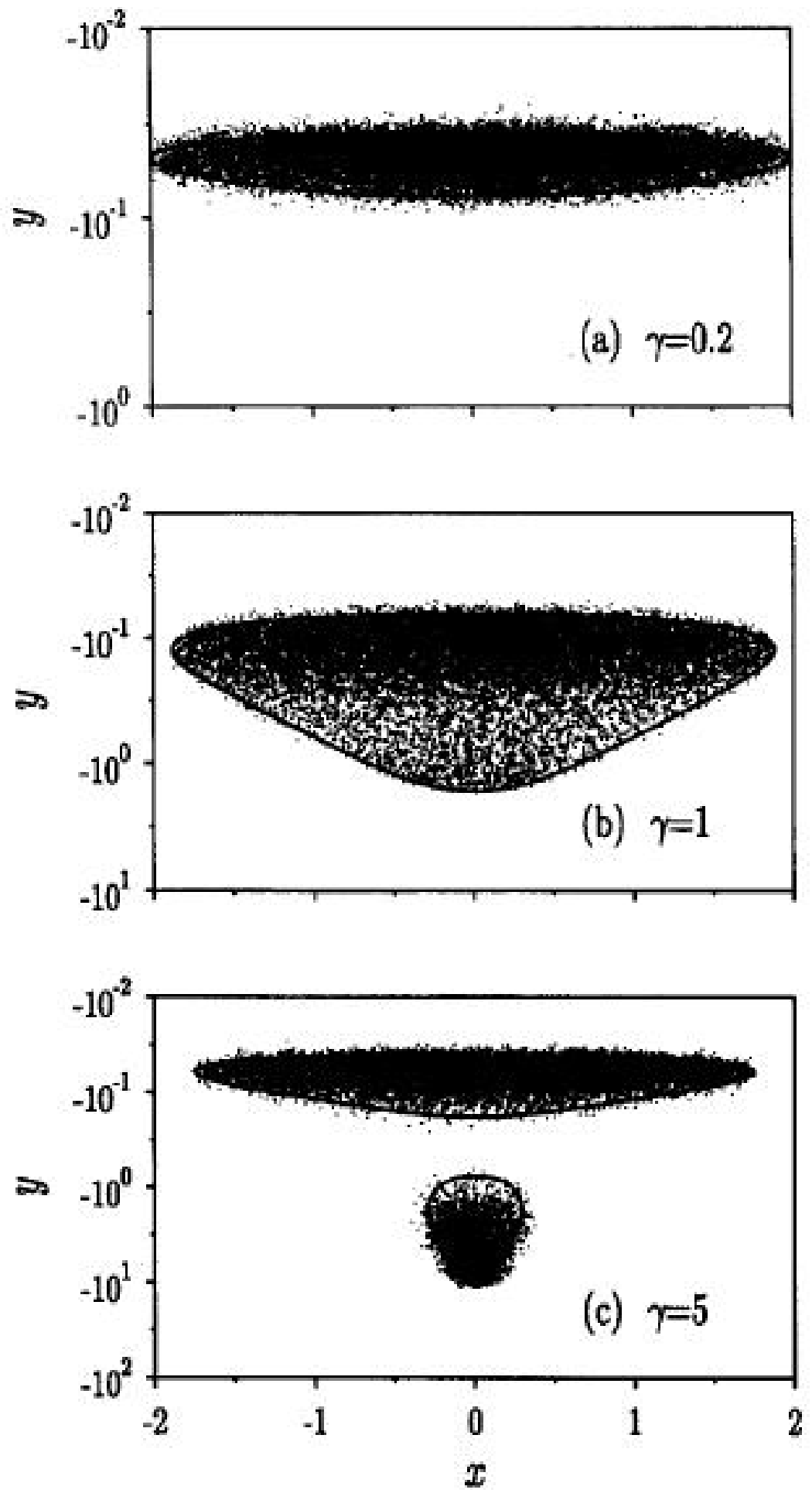

75

Figure 2: Segregation of the cloud of super-radiant states in the complex energy plane $z=x+i y$ as coupling to continuum increases; here $M / N=0.25$ [12]. Note the logarithmic scale on the vertical axis. 

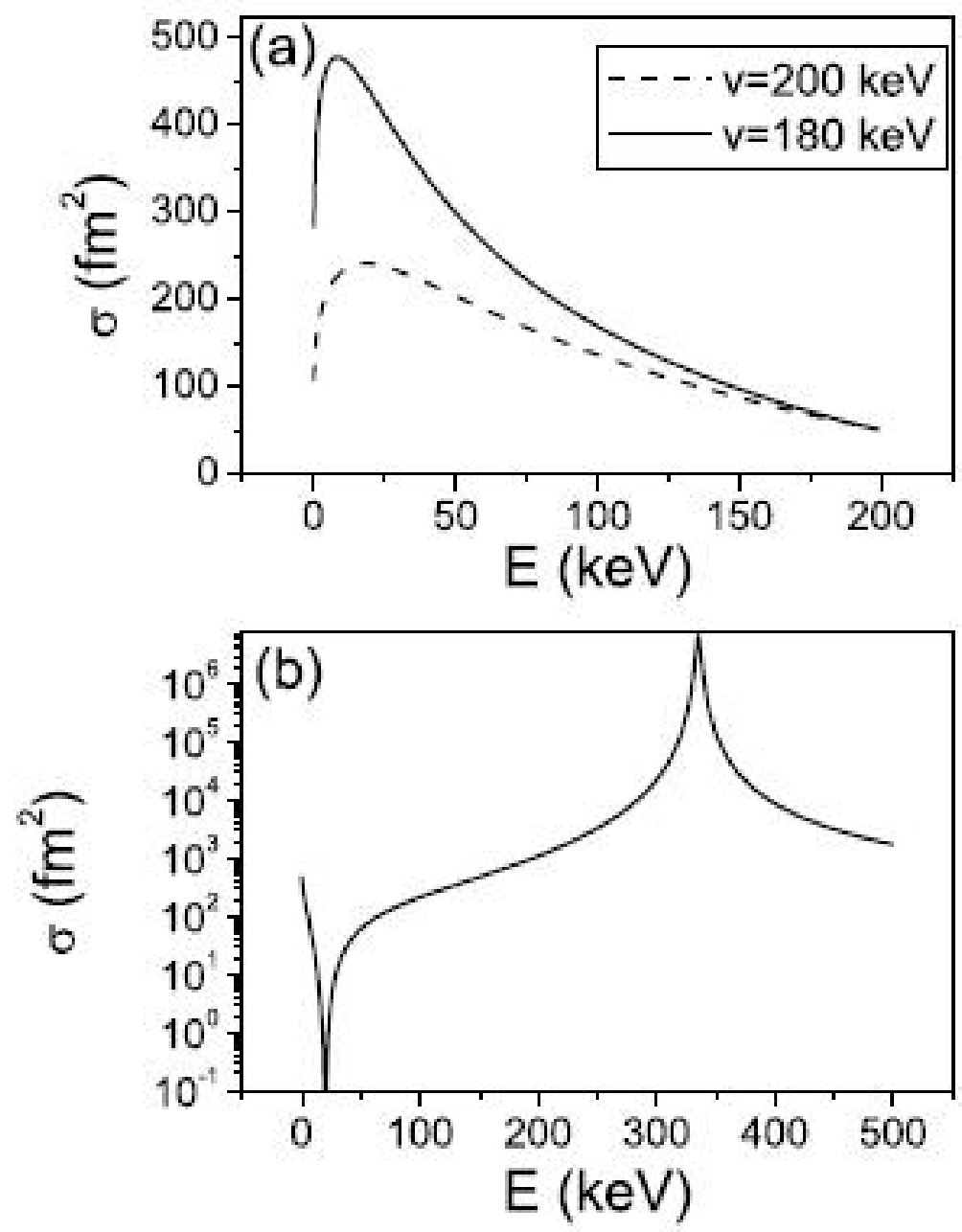

Figure 3: The near-threshold scattering cross section in the two-level model [29], see text. The parameters of eq. 64 are selected from comparison with a corresponding potential model. 


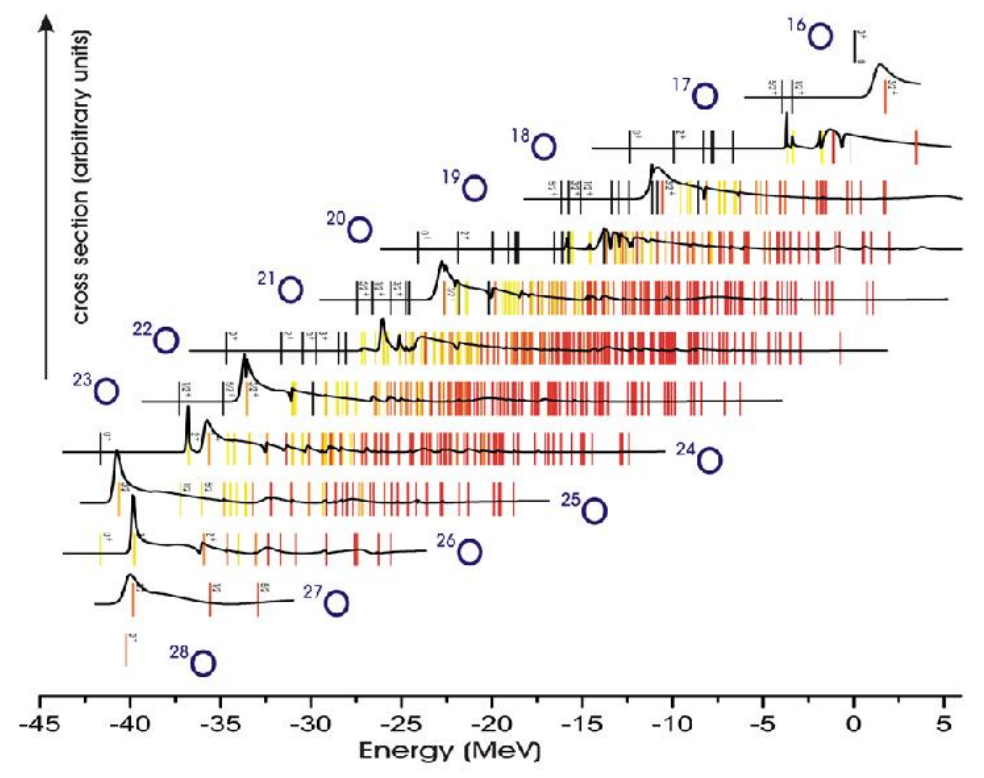

Figure 4: Continuous shell model calculations for the chain of oxygen isotopes [30. Bound states, resonances and cross sections of neutron scattering off the daughter nucleus are consistently calculated in the unified approach. 


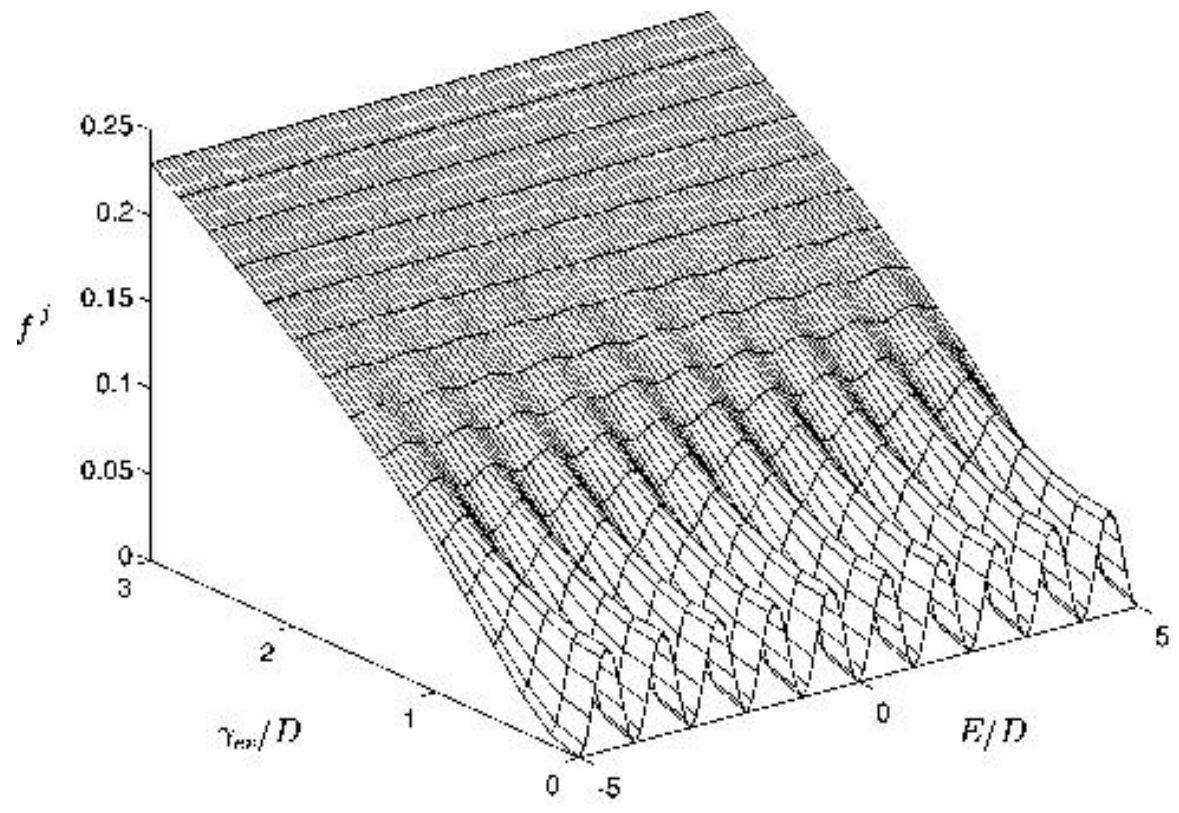

Figure 5: The relative probability of excitation of the doorway state from the continuum channel as a function of energy, $E / D$, and the evaporation width, $\gamma_{\mathrm{ev}} / D$, of the background states [121] for a specific value of the spreading width, $\Gamma^{\downarrow} / D=10$. The oscillations along the energy axis rapidly disappear as $\gamma_{\mathrm{ev}}$ grows. 

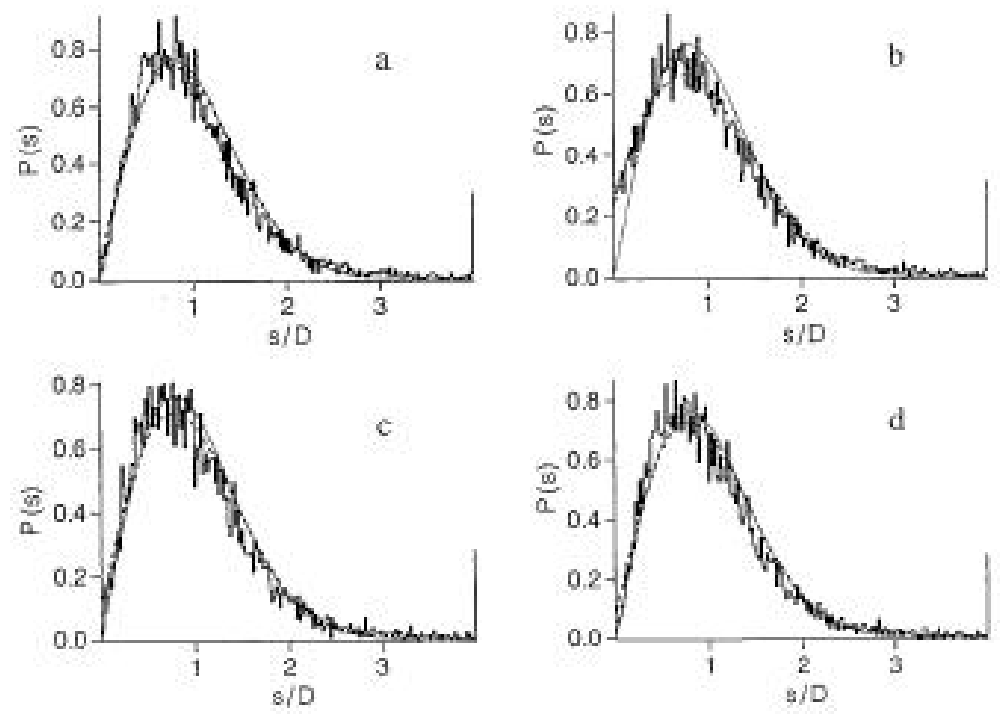

Figure 6: Nearest level spacing distribution found [189] by averaging 50 random matrices $160 \times 160$ with the Gaussian distribution of uncorrelated matrix elements and one open channel. The continuum coupling parameter $\kappa=0.1$ (panel a), 1 (panel b), 5 (panel c), and 50 (panel d). At critical coupling, panel $b$, the level repulsion at short distances disappears and the distribution can be fitted as a combination $\alpha P_{W}(s)+(1-\alpha) P_{G}(s)$ of the Wigner-Dyson distribution $P_{W}(s)$ for the Gaussian orthogonal ensemble (GOE) and the Gaussian distribution $P_{G}(s)$ centered at the mean value of $s$. After the separation of the super-radiant state, the distribution returns to the GOE regime. 

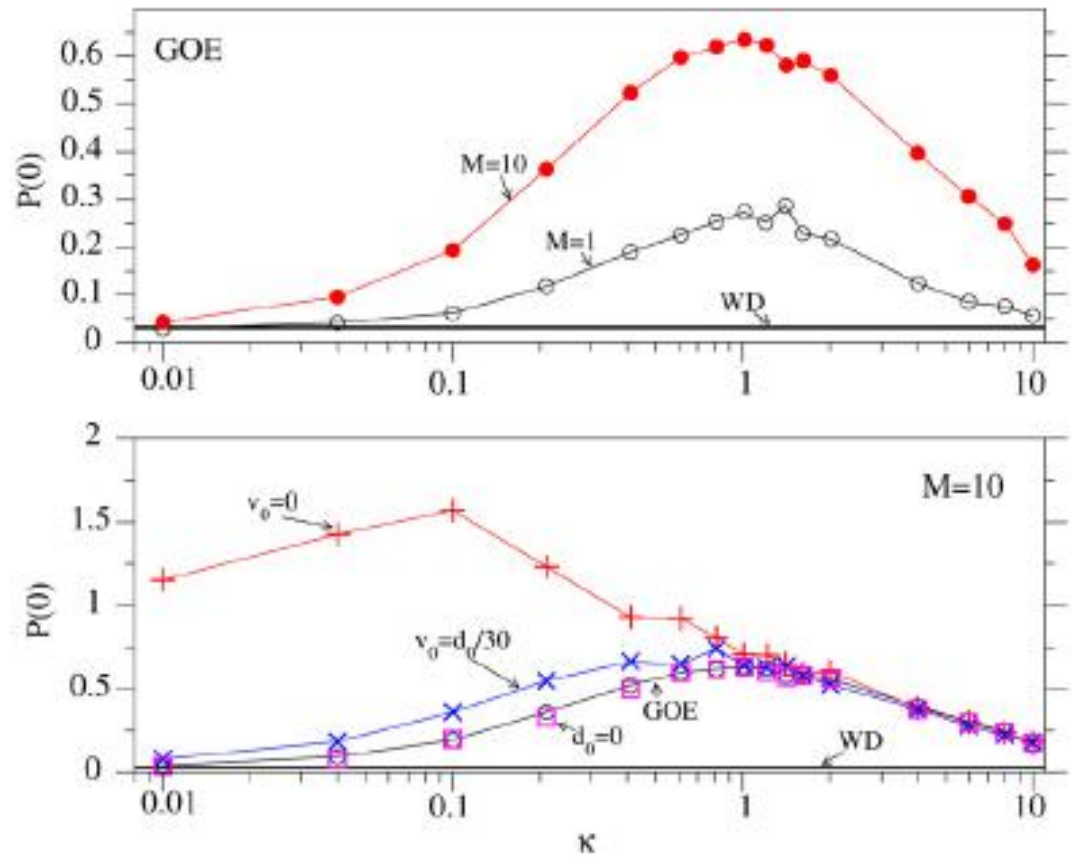

Figure 7: The probability $P(0)$ of finding the resonance energy spacing (in units of the mean spacing) $s<0.04$ [47. The intrinsic dynamics corresponds to the GOE, upper panel, with different numbers of open channels $M$, and to the TBRE, lower panel, for $M=10$, and different strength of the two-body random interaction, from $v=0$ through the critical value for onset of intrinsic chaos to the strong interaction for degenerate single-particle levels, when the results are identical to those for the GOE case. Note that for the TBRE the vertical scale is different; at weak interaction the deviations from $P(0)=0$ start at a very small continuum coupling strength. 

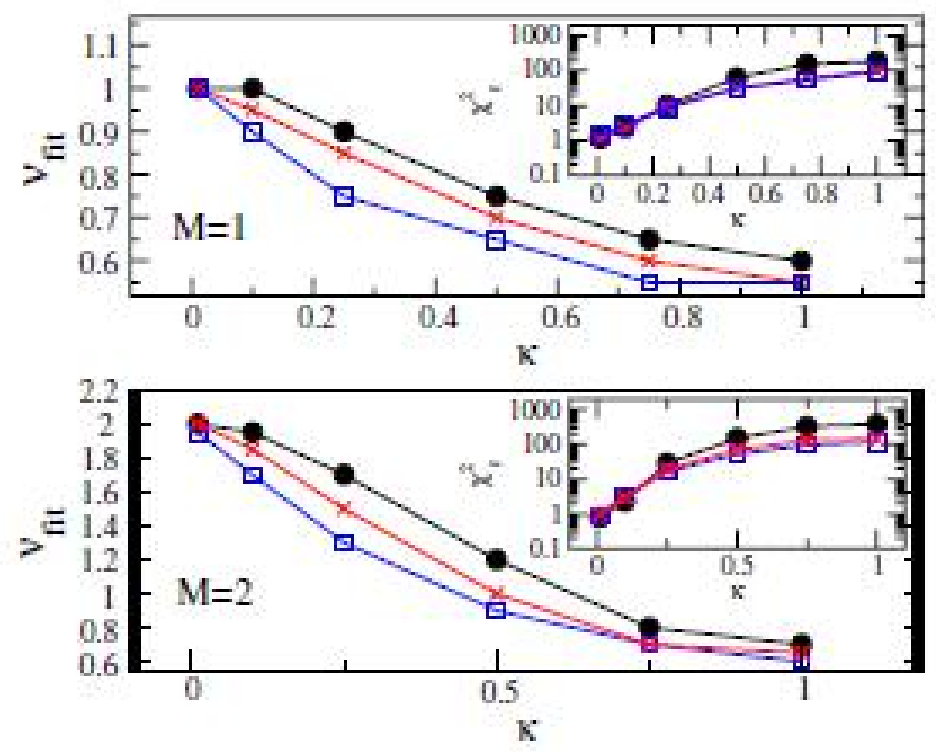

Figure 8: The best fitted parameter $\nu$ for the chi-square distribution with $\nu$ degrees of freedom used for the description of the width distribution found with averaging over 1000 random realizations of intrinsic interactions for one, upper panel, and two, lower panel, open channels [47. Full circles refer to the GOE intrinsic Hamiltonian, crosses stand for the TBRE with the interaction below onset of chaos, and squares for TBRE with no intrinsic chaos. The inserts show the unsatisfactory growing chi-square criterion of the fit as a function of $\kappa$. 


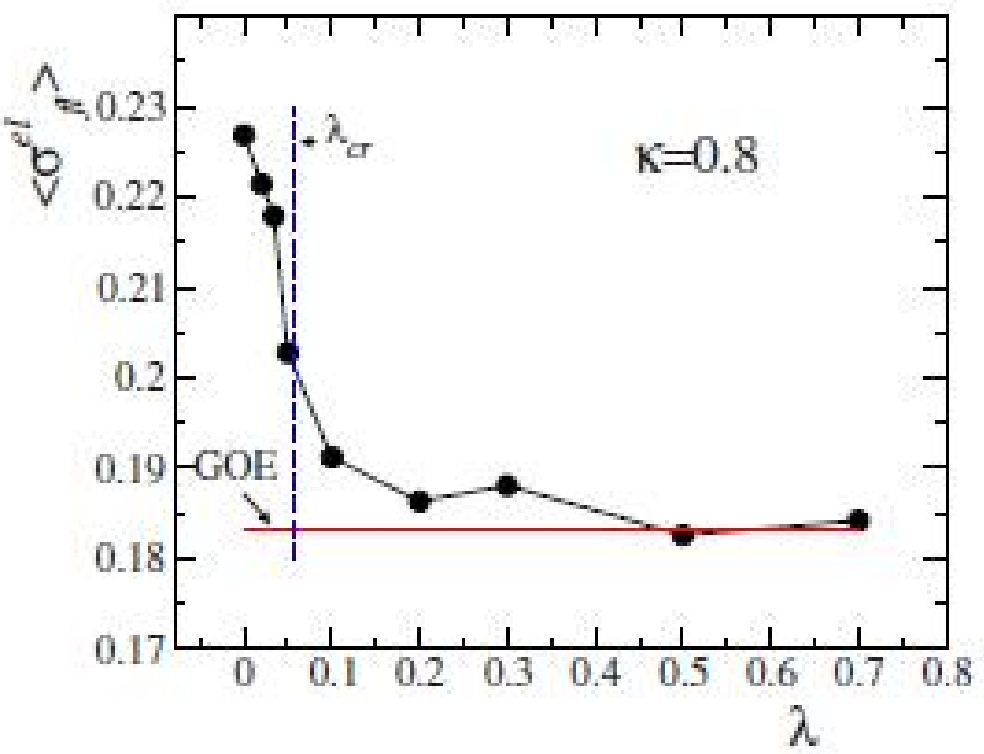

Figure 9: The fluctuating part of the elastic cross section for 10 open channels and $\kappa=0.8$ as a function of the intrinsic interaction strength in the TBRE; the GOE value is shown by a horizontal line [46. The dashed vertical line shows the critical value $\lambda_{\text {cr }}$ for the transition to chaos in the TBRE. 


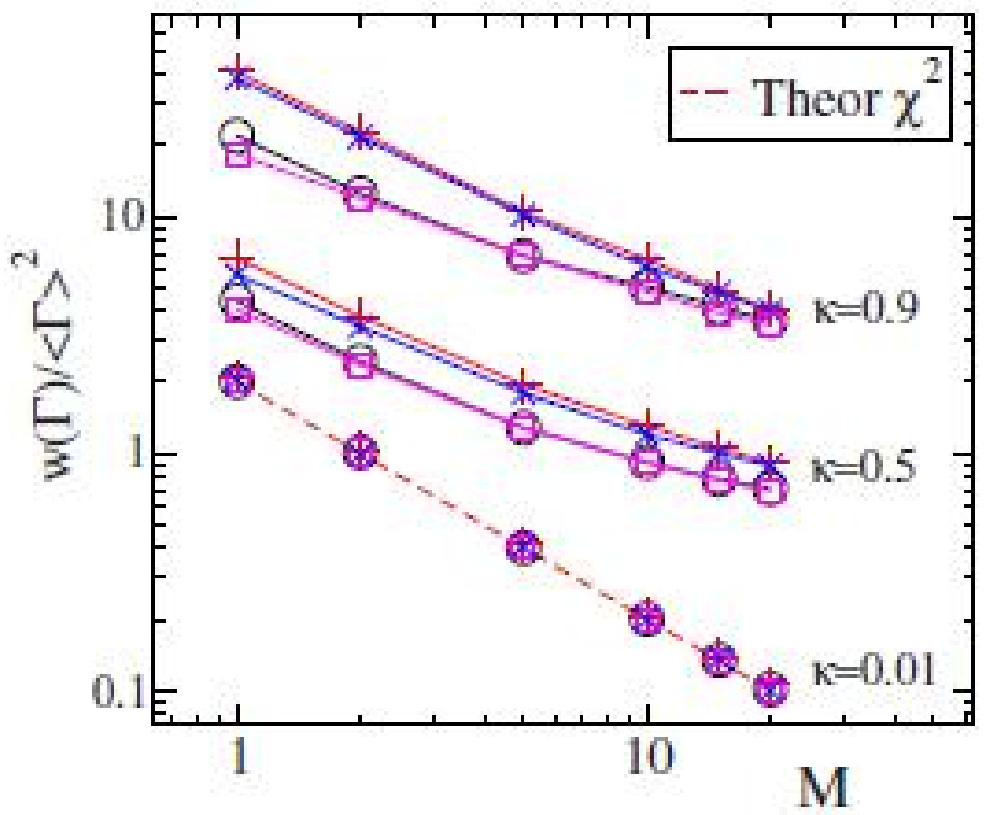

Figure 10: Normalized variance of the width as a function of the number of channels, for different coupling strengths $\kappa$ [46]. Circles refer to the GOE case, pluses to $\lambda=0$, crosses to $\lambda=1 / 30$, and squares to $\lambda \rightarrow \infty$. While for weak coupling, $\kappa=0.01$, the variance decreases with the number of channels very fast in accordance with the expected chi-square distribution (dashed line), for strong couplings, $\kappa=0.5$ and 0.9 , the behavior is different from the $1 / M$ dependence. 


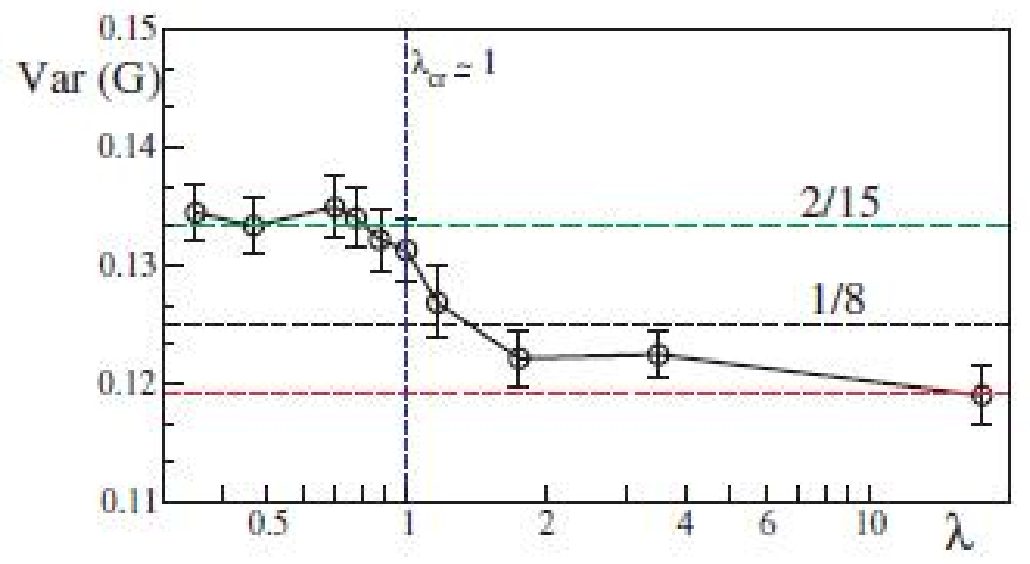

Figure 11: Variance of the conductance, eq. 107), for the TBRE model (7 fermions in 14 orbitals with 20 open channels) as a function of the strength of intrinsic interaction [48. 


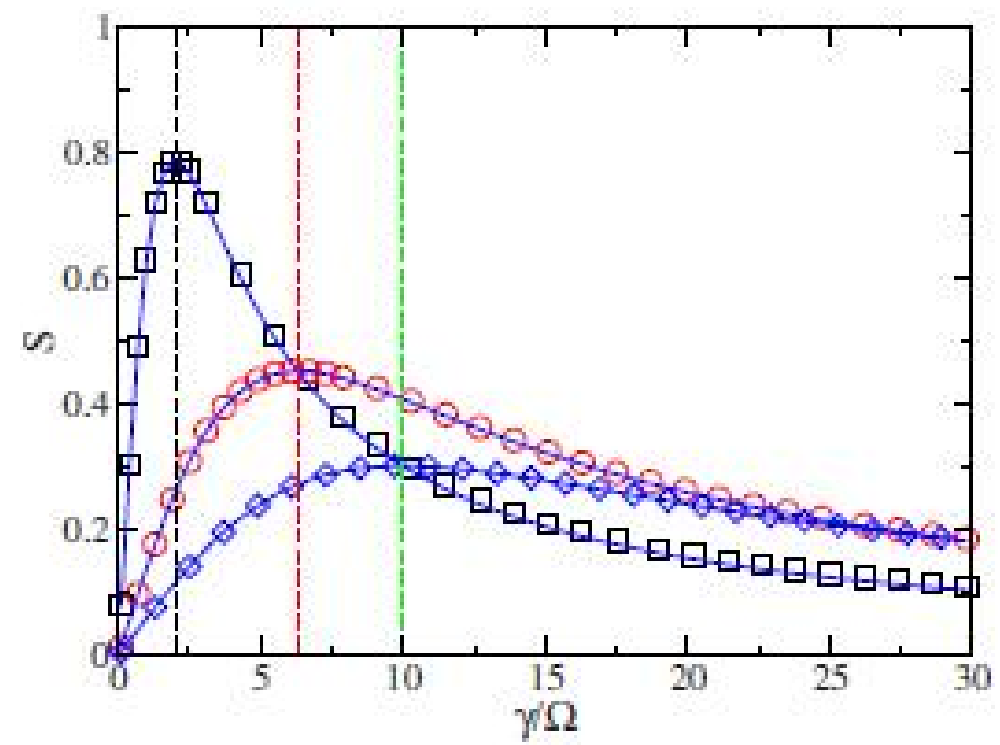

Figure 12: Integrated transmission, eq. (112), for $N=100$ sites and different asymmetry parameters $q$ [50]: squares refer to $q=1$, circles to $q=10$, and diamonds to $q=25$. The vertical dashed lines indicate the critical value of the coupling strength in each case, where the integrated transmission is predicted to have its maximum. 